\title{
LARGE DEFLECTION ELASTIC-PLASTIC ANALYSIS OF CYLINDRICAL SHELLS USING THE FINITE STRIP METHOD
}

\author{
By \\ Pravin Kumar \\ B. Tech. Indian Institute of Technology, New Delhi, 1987
}

\begin{abstract}
A THESIS SUBMITTED IN PARTIAL FULFILLMENT OF
THE REQUIREMENTS FOR THE DEGREE OF

Master of Applied Science
\end{abstract}

in

THE FACULTY OF GRADUATE STUDIES

DEPARTMENT OF CIVIL ENGINEERING

We accept this thesis as conforming

to the required standard

THE UNIVERSITY OF BRITISH COLUMBIA

July 1989

(c) Pravin Kumar, 1989 
In presenting this thesis in partial fulfilment of the requirements for an advanced degree at the University of British Columbia, I agree that the Library shall make it freely available for reference and study. I further agree that permission for extensive copying of this thesis for scholarly purposes may be granted by the head of my department or by his or her representatives. It is understood that copying or publication of this thesis for financial gain shall not be allowed without my written permission.

Department of $C$ ivil Engineering

The University of British Columbia Vancouver, Canada

Date Tuly 18,1989 


\begin{abstract}
A transversely-curved finite strip formulation for the static analysis of circular cylindrical shells is presented. The finite strip analysis is carried out by using one or two analytical modes in the longitudinal direction to determine the response of cylindrical shells subjected to uniform loads. Both linear and quadratic variation of membrane displacements in the curved direction are investigated and it is found that the quadratic variation is far superior.
\end{abstract}

The effect of large deformations is incorporated in the present analysis by including the first order non-linearities in the strain-displacement relations. The material behavior is assumed to be isotropic elastic-plastic. The plasticity portion uses the von-Mises yield criteria and the associated flow rule. For the examples considered, a bi-linear stress-strain relation is assumed. Numerical integration of the virtual work equations is carried out using Gaussian Quadrature. The number of integration points in a given direction is determined by observing the individual terms in the integration or by past experience. The resulting set of non-linear equations is solved iteratively by employing the NewtonRaphson scheme.

Numerical investigations of the method are carried out by modelling cylindrical shells subjected to self-weight or pressure load. These investigations compare the results for laterally loaded cylindrical shells with simply supported or clamped boundaries with analytical and numerical results. The results show that employing one bending mode and one or two membrane modes is sufficient to yield engineering accuracy for design purposes. 


\section{Table of Contents}

$\begin{array}{ll}\text { Abstract } & \text { ii }\end{array}$

List of Tables vii

List of Figures viii

Notations $\quad x$

Acknowledgement $\quad$ xiii

1 Introduction $\quad 1$

2 Methods of Analysis and Literature Review 4

2.1 Analytical Methods of Analysis . . . . . . . . . . . . . . 4

2.1.1 Linear Shell Theory . . . . . . . . . . . . . . . . . 4

2.1.2 Non-Linear Shell Theory . . . . . . . . . . . . . . . . . 11

2.2 Numerical Methods of Analysis . . . . . . . . . . . . . . . . 12

2.2.1 Finite Difference Method . . . . . . . . . . . . . 12

2.2.2 Finite Element Method . . . . . . . . . . . . . . . . . 13

2.3 The Finite Strip Method . . . . . . . . . . . . . . . . . . . 14

3 Mathematical Formulation $\quad 17$

3.1 Introduction . . . . . . . . . . . . . . . . . . . 17

3.2 Finite Strip Discretization . . . . . . . . . . . . 18

3.2.1 Flat Plate Model . . . . . . . . . . . . . . . . 18 
3.2 .2 Curved Shell Model . . . . . . . . . . . . . . . . . . . . . . 19

3.2 .3 Nodal-Line Variables in Finite Strip Models . . . . . . . . . 19

3.3 Displacement Functions . . . . . . . . . . . . . . . . . . 21

3.3 .1 General . . . . . . . . . . . . . . . . . 21

$3.3 .2 \quad$ Flat Plate Strips . . . . . . . . . . . . . . . . 24

3.3 .3 Curved Shell Strip I . . . . . . . . . . . . . . 26

3.3 .4 Curved Shell Strip II . . . . . . . . . . . . . . . 26

3.4 Strain Displacement Relations . . . . . . . . . . . . . . . 27

3.4.1 Flat Plate - Bending Theory . . . . . . . . . . . . 27

3.4 .2 Cylindrical shell - bending theory $\ldots \ldots \ldots \ldots$

3.4 .3 Order of Accuracy Analysis . . . . . . . . . . . . . . . 29

3.5 Constitutive Relations . . . . . . . . . . . . . . 31

3.6 Stiffness Formulation . . . . . . . . . . . . . . . 38

3.6 .1 Flat Plate Element . . . . . . . . . . . . . . . . 38

3.6 .1 .1 Shape Functions $\ldots \ldots \ldots \ldots \ldots$

3.6.1.2 Virtual Work Principle . . . . . . . . . . . 41

3.6 .1 .3 Loa.d Vector . . . . . . . . . . . . . . . . 45

3.6 .2 Curved Shell Strip I . . . . . . . . . . . . . . . 46

3.6.2.1 Shape Functions . . . . . . . . . . . . . 46

3.6.2.2 Virtual Work Principle . . . . . . . . . . . 47

3.6 .2 .3 Load Vector . . . . . . . . . . . . . . . . 48

3.6 .3 Curved Shell II . . . . . . . . . . . . . . . . 49

3.6.3.1 Shape Functions . . . . . . . . . . . . 49

3.6.3.2 Virtual Work Principle . . . . . . . . . . . . 52

3.6.3.3 Load Vector . . . . . . . . . . . . . . . . . . 54

3.7 Newton-Raphson Iterative Procedure . . . . . . . . . . . . . 54 
3.8 Numerical Integration $\ldots \ldots \ldots \ldots \ldots$

3.9 Computer Implementation $\ldots \ldots \ldots \ldots \ldots$

4 Numerical Investigations $\quad 60$

4.1 Introduction . . . . . . . . . . . . . . . . . . 60

4.2 Analysis of a Rectangular Beam . . . . . . . . . . . . . . . 63

4.3 Analysis of the Shell-Roof problem using Flat Plate strips . . . . . . 66

4.4 Analysis of the Shell-Roof problem using Curved Shell strips I . . . . 70

4.5 Analysis of the Shell-Roof Problem using Curved Shell strips II . . . . 75

4.6 Analysis of a Cylindrical Shell Loaded Symmetrically with Respect to Its Axis . . . . . . . . . . . . . . . . . . . . . 79

4.7 Non-Linear Analysis of the Shell-Roof Problem . . . . . . . . . 88

4.7.1 Linear Elastic-Plastic Analysis . . . . . . . . . . . . . 88

4.7 .2 Large Deflection Elastic Analysis . . . . . . . . . . . . 89

4.7.3 Jarge Deflection Elastic-Plastic Analysis . . . . . . . . . . 93

4.8 Clamped Cylindrical Shell - Analysis of the Shell-Roof problem with Clamped boundaries using Curved Shell strips II . . . . . . . . . 97

4.9 Non-Linear Analysis of the Clamped-Shell problem . . . . . . . . . . . 104

4.9.1 Linear Elastic-Plastic Analysis . . . . . . . . . . . . . . . . 104

4.9.2 Large Deflection Elastic Analysis . . . . . . . . . . . 106

4.9.3 Large Deflection Elastic-Plastic Analysis . . . . . . . . . . 106

4.10 Analysis of a Cylindrical Shell Clamped along all Boundaries . . . . . . 109

5 Summary, Conclusions and Suggestions for Future Research 113

5.1 Summary and Conclusions $\ldots \ldots \ldots \ldots \ldots$

5.2 Suggestions for Future Research . . . . . . . . . . . . . . 116 
A Load Vector for Curved Shell II Formulation

B Derivation of the $Q$ matrix

Bibliography 


\section{List of Tables}

2.1 Gaussian Curvature .......................... 5

4.1 Linear Elastic Response of a Simply Supported Beam . . . . . . . . . . 65

4.2 Linear Elastic Response of the Shell-Roof Problem Using Flat Strips . . : 68

4.3 Analysis of membrane and bending stresses in the Shell-Roof Problem Using Flat Plate strips . . . . . . . . . . . . . . . . . . . . . 69

4.4 Linear Elastic Response of the Shell-Roof Problem Using Curved Shell

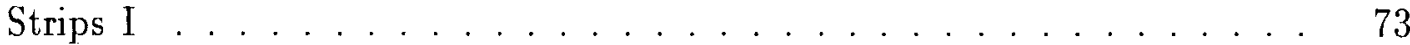

4.5 Linear Elastic Response of the Shell-Roof Problem Using Curved Shell

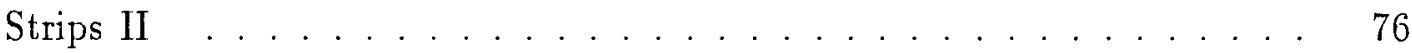

4.6 Analysis of membrane and bending stresses in the Shell-Roof Problem Using Curved Shell strips IJ . . . . . . . . . . . . . . . . . 77

4.7 Linear Elastic Response of an Axisymmetric Cylindrical Shell Using Curved

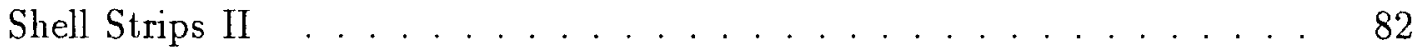

4.8 Linear Elastic Response of the Clamped Shell Problem Using Curved Shell Strips II . . . . . . . . . . . . . . . . . . . . . 102 


\section{List of Figures}

2.1 Longitudinal Boundary Conditions for Cylindrical Shells . . . . . . . 7

3.1 Flat Plate Discretization . . . . . . . . . . . . . . 18

3.2 Curved Shell Discretization _. . . . . . . . . . . . . . . 20

3.3 A Flat Plate Element . . . . . . . . . . . . . . . . . 21

3.4 Curved Shell Element I . . . . . . . . . . . . . . . . . 22

3.5 Curved Shell Element II . . . . . . . . . . . . . . . . . . 23

3.6 Stress-Strain relationship . . . . . . . . . . . . . . . . 32

3.7 Hardening Models . . . . . . . . . . . . . . . . . . . . 35

3.8 Stress-plastic strain Relationship . . . . . . . . . . . . . . 36

4.1 Cross-Section of the Simply Supported Beam . . . . . . . . . . . . . . 64

4.2 Shell-Roof problem ... . . . . . . . . . . . . . . . 66

4.3 Vertical Displacement of the free edge . . . . . . . . . . . . 71

4.4 Relative Error in Strain Energy using Curved Shell strips I . . . . . . . . 74

4.5 Relative Error in Strain .Energy Using Curved Shell strips II . . . . . . . 78

4.6 Axisymmetric Cylindrical Shell . . . . . . . . . . . . . . 79

4.7 Radial Displacement . . . . . . . . . . . . . . . . . 84

4.7 Radial Displacement . . . . . . . . . . . . . . . . . . . . 85

4.8 Moment Distribution . . . . . . . . . . . . . . . . . . 86

4.8 Moment Distribution . . . . . . . . . . . . . . . . . . 87

4.9 Vertical Displacement of the free edge . . . . . . . . . . . . . . . . 90 
4.10 Variation of Work Done by the Load for the Shell-Roof Problem . . . . 91

4.11 Vertical Displacement of the free edge . . . . . . . . . . . . . . 92

4.12 Variation of Strain Energy for the Shell-Roof problem . . . . . . . . . 9 94

4.13 Vertical Displacement of the free edge . . . . . . . . . . . 95

4.14 Variation of Work Done by the Load for the Shell-Roof problem . . . . 96

4.15 Clamped Shell problem _. . . . . . . . . . . . . 97

4.16 Vertical displacement of the free edge . . . . . . . . . . . . . 101

4.17 Variation of $v$ and $w$ displacements for the Clamped-Shell Problem . . 105

4.18 Vertical Displacement of the free edge . . . . . . . . . . . . . 107

4.19 Vertical Displacement of the free edge . . . . . . . . . . . . 108

4.20 Clamped-Clamped Shell . . . . . . . . . . . . . . 110

4.21 Vertical Displacement of the crown . . . . . . . . . . . . . 111 


\section{Notations}

$R_{X} \quad$ Radius of Curvature in the $X$-direction

$R_{Y} \quad$ Radius of Curvature in the $Y$-direction

X Global longitudinal direction

$Y, \phi \quad$ Global transverse/ tangential direction

Z Global normal or vertical direction

$L \quad$ Length of the element/member in the $X$-direction

$t \quad$ Thickness of the member

$\sigma_{X} \quad$ Normal stress in the $X$-direction

$\sigma_{\phi} \quad$ Normal stress in the $Y$-direction

$\sigma_{Z} \quad$ Normal stress in the $Z$-direction

$Q_{x} \quad$ Transverse shear along the edge $x=$ constant

$u \quad$ Displacement component along the $X$ direction

$v \quad$ Displacement component along the $Y$ direction

$w \quad$ Displacement component along the $Z$ direction

$N_{x} \quad$ Membrane normal force along the $X$ direction

$N_{\phi} \quad$ Membrane normal force along the $\phi$ direction

$N_{x \phi}, N_{\phi x} \quad$ Membrane shearing force

$M_{x} \quad$ Bending Moment about $Y$-axis

$M_{\phi} \quad$ Bending Moment about $X$-axis

$M_{x \phi}, M_{\phi x} \quad$ Twisting Moments 
$\theta \quad$ Rotation about the $X$-axis

$b \quad$ Width of the element

$\beta \quad$ Angular quantity proportional to the distance along the tangential direction

$\xi \quad$ Non-dimensional $X$ co-ordinate

$\eta \quad$ Non-dimensional $Y$ co-ordinate

$g_{m}^{u}(\xi) \quad$ Longitudinal mode shape for $u$ displacement

$g_{n}^{\prime \prime}(\xi), g_{k}^{\prime \prime}(\xi) \quad$ Longitudinal mode shape for $v$ displacement

$g_{p}^{w}(\xi) \quad$ Longitudinal mode shape for $w$ displacement

$\phi(\xi) \quad$ Clamped beam vibration mode

$\epsilon_{x} \quad$ Normal strain in $X$ direction

$\epsilon_{y} \quad$ Normal strain in $Y$ direction

$\epsilon_{x y} \quad$ Shear strain

$R \quad$ Radius of Curvature

$U \quad$ Strain Energy of the cylindrical shell in bending

$D \quad$ Rigidity Modulus

$\nu \quad$ Poisson's ratio

$u_{x} \quad$ Derivative of $u$ with respect to $x$

$u_{y} \quad$ Derivative of $u$ with respect to $y$

$v_{x} \quad$ Derivative of $v$ with respect to $x$

$v_{y} \quad$ Derivative of $v$ with respect to $y$

$w_{x x} \quad$ Double Derivative of $w$ with respect to $x$

$w_{y y} \quad$ Double Derivative of $w$ with respect to $y$

$w_{x y} \quad$ Double Derivative of $w$ with respect to $x$ and $y$

$O(l) \quad$ Order of $l$ 
$\sigma_{o} \quad$ Uniaxial yield stress

$\epsilon_{o} \quad$ Uniaxial yield strain

E Modulus of Elasticity

$E_{T} \quad$ Tangent modulus

$\epsilon_{e} \quad$ Elastic strain

$\epsilon_{p} \quad$ Plastic strain

$U_{d} \quad$ Distortion strain energy

$G \quad$ Shear modulus

$J_{2} \quad 2^{\text {nd }}$ invariant of the stress deviator

$\sigma_{1} \quad$ Principal stress

$\sigma_{2} \quad$ Principal stress

$\sigma_{3} \quad$ Principal stress

$F \quad$ Yield Function

$N_{i}^{u} \quad$ Shape functions for the variation of $u$

$N_{i}^{v} \quad$ Shape functions for the variation of $v$

$N_{i}^{w} \quad$ Shape functions for the variation of $w$

$[B] \quad$ Linear strain-displacement matrix

$[C] \quad$ Non-Linear strain-displacement matrix

$\left[T_{e}\right] \quad$ Element transformation matrix

P Global Load vector

$p_{e} \quad$ Element load vector

Toler $_{1} \quad$ Acceptable tolerance in Maximum norm criterion

Toler $_{2}$ Acceptable tolerance in Euclidean norm criterion 


\section{Acknowledgement}

The author wishes to thank Dr. M.D.Olson and Dr. D.L.Anderson for their advice and guidance throughout the course of this research and in the preparation of this thesis. The author is also appreciative of the encouragement offered by all his friends, particularly J.D.Dolan and T.S.Koko, in the preparation of this thesis.

Financial support in the form of Research Assistantship from the Department of Civil Engineering, University of British Columbia, is gratefully acknowledged. 
To

My Parents

Phool and Madan Mohan Arora 


\section{Chapter 1}

\section{Introduction}

The study of three-dimensional continua constitutes one of the very important departments in the theory of solid mechanics. With rational simplifications to the threedimensional theory of elasticity, the engineering theories of medium-thin plates and of thin-shells may be derived and applied to a large class of engineering problems distinguished by a characteristically small dimension in one direction. These theories find wide application in construction practice, aviation, shipbuilding; machine manufacture and other fields of technology.

It is of present as well as historical interest to recognize that significant structures were constructed utilizing the effective load resisting mechanism of doubly curved structural members e.g. domes in ancient times. The Pantheon of ancient Rome; constructed of cementitious material, has stood for about two thousand years; beautifully tile-covered mosques from the Persian Empire survive in Iran. Also, St. Paul's Cathedral remains to grace the London skyline.

Perhaps one reason that several shell structures remain from antiquity is the ability of surface structures to survive extreme loading. It was reported that a cooling tower shell was among the few surviving structures in the Tanjung, China earthquake of 1976. A hyperbolic paraboloid resisted the Mexico city earthquake of 1985 without apparent structural damage amid totally destroyed conventional structures. ·

Structurally, a shell may be termed a two-dimensional extensional member. Shells 
differ from plates in its primary load carrying mechanism. By virtue of its curvature, the shell can develop thrusts to form the primary load resisting mechanism in addition to the transverse shears and the bending and twisting moments present in the plate. A combination with respect to the plate and the shell which is of considerable practical interest and is dealt with in the following chapters, is a form curved in one direction such as a cylinder. Although, even the so-called developable surface is generally regarded as a shell, the resisting mechanics in the uncurved direction is basically flexural, whereas in the curved direction it may be extensional.

The complicated load-resisting mechanism associated with the interaction of flexural and the membrane deformations makes the analysis of cylindrical shells a complex procedure. The analytical solutions used in the earlier analysis suffer with limitations in their scope and accuracy. Finite element procedures have been formulated for the analysis of cylindrical shells with various boundary conditions and subjected to various types of loading - static and dynamic. In the recent past, efforts have been made to simplify the finite-element technique to model plates [40] and shells to reduce the computational effort while still maintaining engineering accuracy and versatility of the finite-element method.

The Finite Strip method satisfies these requirements. Numerous programs have been developed for the analysis of beams, plates, stiffened panels and to a limited extent, cylindrical shells. The finite strip method can be used with greater efficiency in the analysis of structures with a regular geometric plan and simple boundary conditions, where a finite element procedure would be both cumbersome and expensive. The primary objective of the present study is to investigate the applicability of the finite strip method to the analysis of cylindrical shells.

In the first stage of the investigation, a linear elastic analysis of laterally loaded cylindrical shells is carried out. The deffection of the cylindrical shell for such an analysis 
is generally small relative to its thickness. However, as the deflections in the structure increase, membrane forces become significant and this is accompanied by the stretching of the middle plane. The structure may then undergo plastic deformations which may exceed the safe design limits. This is a major concern for the structural engineer who is always on the lookout for an effective numerical procedure which can accurately predict the deformation profiles and stress distribution throughout the structure.

In the present study, a complete non-linear analysis of cylindrical shells is carried out by incorporating both geometric and material non-linearities. Finally, the accuracy of the finite strip solution is tested by employing a single bending mode with one or two membrane modes in the finite strip modelling of cylindrical shells. The finite strip solution, thus obtained, provides a reasonable estimate of deflections and stresses which can then be used by the design engineer.

Chapter 2 of this thesis covers the analytical techniques which have been used to analyze cylindrical shells. The finite element modelling of shells for geometric and material non-linearity is also discussed. Finally, the use of the finite strip method in the analysis of cylindrical shells is reviewed.

Chapter 3 deals with the general problem of the analysis of cylindrical shells. The mathematical formulation of the finite strip problem is discussed in detail. Various aspects of computer implementation are also discussed. The Newton-Raphson iterative scheme and the method of numerical integration is also highlighted.

Chapter 4 includes the numerical investigations carried out for several example problems and a complete comparison with analytical or numerical procedures wherever possible.

Chapter 5 provides a summary and lists the conclusions drawn from the present study. Suggestions for extending the proposed numerical procedure to other problems in structural engineering are also included. 


\section{Chapter 2}

\section{Methods of Analysis and Literature Review}

\subsection{Analytical Methods of Analysis}

\subsubsection{Linear Shell Theory}

Recall that a cylindrical shell could be derived from a plate by forming the middle plane to a singly curved surface which is circular in cross-section. For a shell of constant thickness, the middle plane is defined to be one which lies mid-way between the two curved surfaces.

Another definition with respect to the shell geometry, which is referred in the later sections, is Gaussian Curvature. The Gaussian curvature of a shell is dependent on shell geometry. For a general shell curved in the two principal directions $X$ and $Y$, the Gaussian Curvature is defined by the fraction $\frac{1}{R_{X} R_{Y}}$ where $R_{X}$ and $R_{Y}$ are the curvatures in the principal directions.

If we consider the normal sections corresponding to the principal directions in a general shell, the Gaussian curvature could be positive, negative or zero. If the centers of curvature in both the principal directions lie on the same side of the surface, the Gaussian curvature is positive and is negative if the centers lie in opposite directions. 
If one of the radii of curvature is equal to infinity the Gaussian curvature is zero. The above information is summarized in Table 2.1. A flat plate is a special case of a shell with zero Gaussian curvature since both radii are infinite.

Table 2.1: Gaussian Curvature

\begin{tabular}{|c|c|c|c|}
\hline Classification & Positive & Negative & Zero \\
\hline Surface & Doubly Curved & Doubly Curved & Singly Curved \\
Examples & Sphere & $\begin{array}{c}\text { Ayperboloid of } \\
\text { Revolution } \\
\text { Anticlastic }\end{array}$ & Cylinder \\
Developability & Nondevelopable & Nondevelopable & Developable \\
$\begin{array}{c}\text { Type of Governing } \\
\text { Differential Equation }\end{array}$ & Elliptic & Hyperbolic & Parabolic \\
\hline
\end{tabular}

The mathematical formulation of the linear theory of cylindrical shells is based on the three-dimensional theory of elasticity. In developing the theory of thin shells, considerable simplification is accomplished by reducing the analysis of cylindrical shells to the study of the deformations of the middle surface. The complicated equations of equilibrium, based on three-dimensional theory, involving three space variables reduces to a system involving two space variables defined at the middle plane of the shell. Further simplifications to the governing equations of equilibrium lead to the formulation of the membrane theory of shells.

The membrane or the 'momentless' theory of shells, as the name suggests, assumes that shells primarily resist loads by extension or stretching of the middle plane. The membrane theory was first postulated by Lamé and Clapeyron in the early nineteenth century. The main motivation towards the formulation of the membrane theory was to 
make the analysis of shells a determinate problem. The theory yielded deflections and in-plane forces which gave a reasonable estimate of the deformation pattern and the internal forces developed in the shell.

The membrane theory, apparently, can only be used to analyze shells which behave like a membrane. The shell is considered to behave like the membrane if the bending strains are zero or negligible as compared with the axial strains. The shells which fall in this category include:

1. Flexible shells or membranes, which by virtue of their thinness have negligible bending stiffness and as a result are incapable of resisting bending.

2. Shells that have a finite bending stiffness but they do not develop significant bending strains depending on the loading and support conditions.

The state of stress in a membrane is 'momentless' if the bending stiffness is negligible.

A cylindrical shell supported by a diaphram on the curved boundaries and supported along the longitudinal boundaries, subjected to a uniform pressure falls into the second category. The situation is illustrated in Fig.2.1 where it can be seen that the cylindrical shell resists transverse loading primarily as a series of arches, which is an implication of membrane theory. On the other hand, depending on the length of the shell and the longitudinal boundary conditions, e.g. in case of a short shell with free longitudinal boundaries, the behavior of the shell resembles that of a beam. As a result, the primary load resisting mechanism is flexure.

Vlasov [1] postulated that the membrane theory yielded a complete finite solution for shells with positive Gaussian curvature. However, in case of shells with negative curvature, the theory is applicable only in those cases of loading for which the hyperbolic equations corresponding to these shells yield completely defined finite values for the internal forces. 


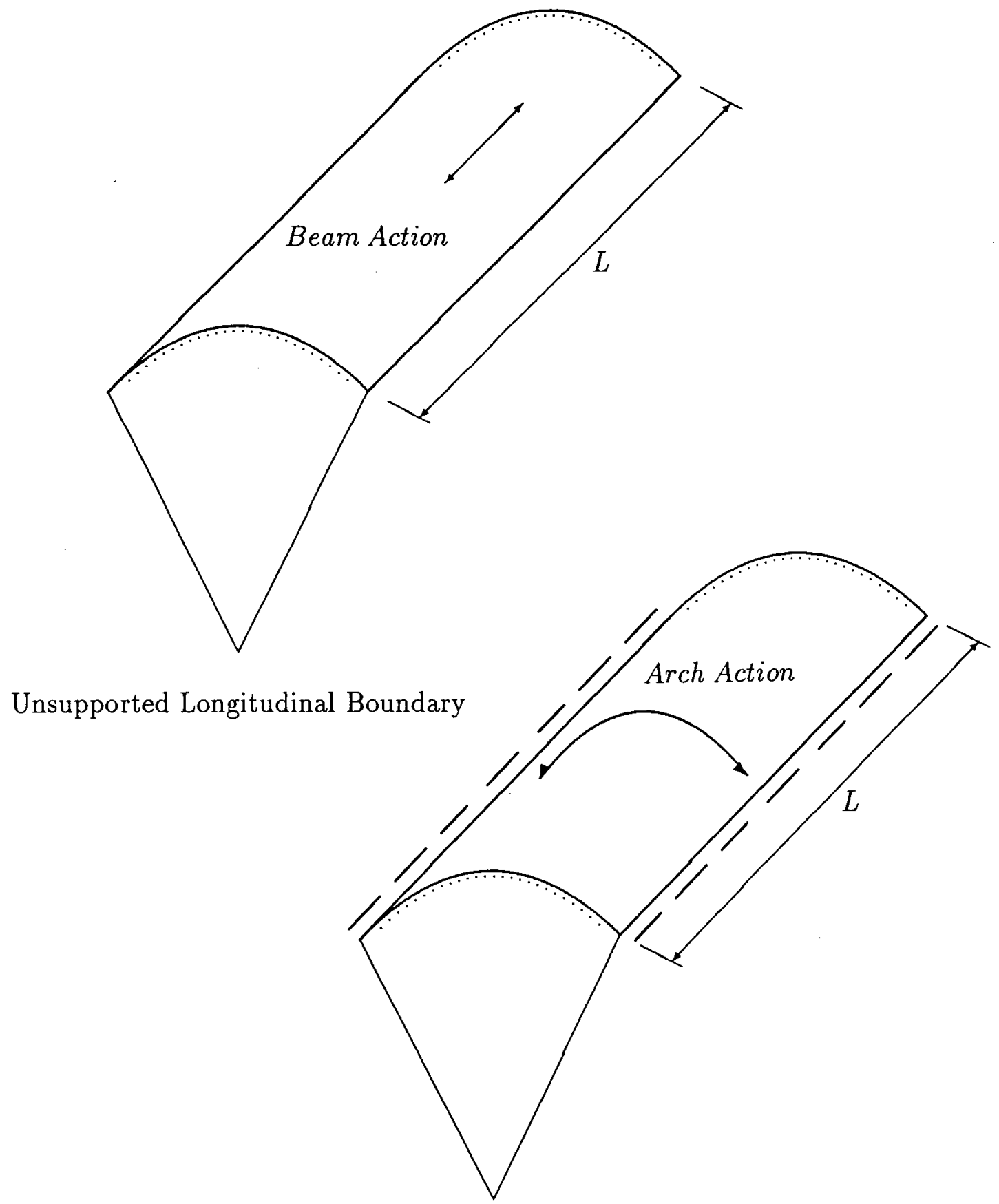

Supported Longitudinal Boundary

Figure 2.1: Longitudinal Boundary Conditions for Cylindrical Shells 
Given the limitations imposed on the behavior of the shell, its geometry, the type of loading and the constraints on the boundary conditions, the need for a general theory of shells lead to a systematic approach towards the development of the 'bending' theory of shells.

The simplified bending theories of shells are (in general) based on Love's approximations. To simplify the strain-displacement relations, Love hypothesized the first-order theory. The assumptions underlying the hypothesis are:

1. The shell thickness, $t$, is negligible as compared with the least curvature, $R_{\text {min }}$, of the middle surface i.e. $\mathrm{t} / \mathrm{R}_{\min } \ll 1$.

2. All points normal to the middle surface of the undeformed shell remain normal after deformation.

3. For all kinematic relations, the distance $z$ of a point from the middle surface of the shell may be considered unaffected by the deformation of the shell.

4. The component of stress normal to the middle surface $\sigma_{Z}$, may be considered negligible as compared with the stress components in the longitudinal and transverse directions, $\sigma_{X}$ and $\sigma_{\phi}$ respectively.

5. Strains and displacements are small so that the quantities containing second-and higher-order terms are neglected in the strain displacement equations.

The assumptions stated above, when applied to a real shell imply that the transverse shear strains $\gamma_{x z}$ and $\gamma_{y z}$ are smaller than the extensional and in-plane shearing strains. Secondly, the strain and the stress in the $Z$-direction is negligible, where $X$ and $Y$ are the longitudinal and the transverse axes of the shell respectively and the $Z$-axis is perpendicular to the $X Y$ plane. Obviously, this assumpiion would be valid for thin 
shells. The governing differential equations for the analysis of circular cylindrical shells were derived by Love [2], Flügge [3] and Timoshenko [4] based on the moment theory.

The second-order approximation theory of Flügge $[3]$ retains the $\frac{z}{R}$ term in the stressresultant equations and in the strain-displacement relations. The equilibrium equations obtained by Flügge for a general shell were discussed by Kempner [42], who obtained them as a special case of a unified shell theory.

Second-order approximation equations were derived by Vlasov [1] directly from the three-dimensional linear elasticity equations for a thick shell. The assumptions $\epsilon_{\bar{z}}=$ $\gamma_{\phi z}=\gamma_{x \phi}=0$ is made, where $\gamma_{\phi z}$ and $\gamma_{x \phi}$ are the transverse shear strains and $\epsilon_{z}$ is the normal strain. The assumptions of zero normal strain and zero transverse strains permits a rapid transition from the three-dimensional theory to the two-dimensional equations of shell-theory, but it should not be interpreted in its strict sense as implying a state of plane strain. Rather, it is a convenient assumption equivalent to the basic Kirchhoff-Love hypothesis that normal lines remain normal and their extensions are negligible [43].

In deriving the three governing differential equations for the linear elastic analysis of thin cylindrical shells, Timoshenko [4] assumed that the effect of the change in curvature of the shell element to bending of the element was negligible and therefore, could be neglected. However, if the in-plane forces $N_{x}, N_{y}$ and $N_{x y}$ were comparable with the critical values at which lateral buckling of a shell may occur, this effect would be significant and hence, the corresponding terms in the equilibrium equations had to be retained. Further simplifications to the derivation was achieved by disregarding the terms of $O\left(\frac{t^{2}}{R^{2}}\right)$. This assumption, though not fully justified, was found to give good results for a wide class of problems [39]. The suppression of the $O\left(\frac{t^{2}}{R^{2}}\right)$ terms is equivalent to neglecting the influence of stress couples on the in-plane equilibrium equation. The final set of simplified system of equations used in the analysis of thin cylindrical shells could be written as [4]: 


$$
\begin{gathered}
\frac{\partial^{2} u}{\partial x^{2}}+\frac{1-\nu}{2 R^{2}} \frac{\partial^{2} u}{\partial \phi^{2}}+\frac{1+\nu}{2 R} \frac{\partial^{2} v}{\partial x \partial \phi}-\frac{\nu}{R} \frac{\partial w}{\partial x}=0 \\
\frac{1+\nu}{2} \frac{\partial^{2} u}{\partial x \partial \phi}+R \frac{1-\nu}{2} \frac{\partial^{2} v}{\partial x^{2}}+\frac{1}{R} \frac{\partial^{2} v}{\partial \phi^{2}}-\frac{1}{R} \frac{\partial w}{\partial \phi}=0 \\
\nu \frac{\partial u}{\partial x}+\frac{\partial v}{R \partial \phi}-\frac{w}{R}-\frac{t^{2}}{12}\left(R \frac{\partial^{4} w}{\partial x^{4}}+\frac{2}{R} \frac{\partial^{4} w}{\partial x^{2} \partial \phi^{2}}+\frac{\partial^{4}}{R^{3} \partial \phi^{4}}\right)==-\frac{R p}{\left(1-\nu^{2}\right)} \frac{1}{E t}
\end{gathered}
$$

where $u, v$ and $w$ are the displacement components in the directions $x, \phi$ and $z$ respectively, $\nu$ is the Poisson's ratio, $E$ is the modulus of elasticity, $R$ and $t$ are the radius of curvature and the thickness of the shell respectively and $p$ is the lateral load on the shell. In this set of governing differential equations, $x$ defines the longitudinal axis of the shell, $\phi$, the transverse axis and $z$ is oriented perpendicular to the $x-\phi$ plane.

The resulting system of differential equations are the basic solvable equations for circular cylindrical shells. To solve the differential equations, four boundary conditions should be specified at each point on the edge of the middle plane. For most common boundary conditions i.e simply supported, clamped and free, four conditions need to be specified along each edge $x=$ constant and $y=$ constant. They are given by:

1. Simply Supported Edge. Such a hinged edge is not able to transmit a moment $M_{x}$ needed to enforce the condition $\frac{\partial w}{\partial x}=0$. Assuming also that there is no edge resistance in the direction $x$, we arrive at the boundary conditions:

$$
v=0, \quad w=0, \quad M_{x}=0 \quad N_{x}=0
$$

2. Built-in Edge. Usually such a support is considered as perfectly rigid, and the edge conditions then are:

$u=0, \quad v=0, \quad w=0 \quad \frac{\partial w}{\partial x}=0$ 
3. Free Edge. Letting all the stress resultants vanish on the edge, we find that the conditions characterizing the free edge assume the form:

$$
N_{x}=0, \quad M_{x}=0, \quad S_{x}=0 \quad T_{x}=0
$$

where $S_{x}=N_{x \phi}+\frac{M_{x, \phi}}{R}$

and $T_{x}=Q_{x}-\frac{\partial M_{x \phi}}{R \partial \phi}$

\subsubsection{Non-Linear Shell Theory}

The linear shell theory discussed in Section 2.1.1 is based on Hooke's law and the omission of non-linear terms in both the equations for strain components and equilibrium equations. Once the deflections or the strains become large or it is desired to investigate buckling, then non-linear theories are necessary [43].

Most of the derivations of shell theories valid for arbitrary large deformations [5] are based on the adoption of Kichhoff-Love hypothesis and are formulated in terms of the two-dimensional force and moment resultants using variational methods. Tene and Epstein [6] used the exact three-dimensional variational expression and used a kinematic approach in the analysis. Using Ritz procedure, a set of equations was obtained in terms of kinematic unknowns without any reference to quantities like the shear forces and bending moments. The development of a variational approach provided the basis for a stiffness formulation which has been extensively used in numerical procedures like the finite element and the finite strip methods. Wempner [7] derived an approximate method for the analysis of elastic-plastic shells. He hypothesized that an inner layer is in an elastic state and one or two outer layers in plastic states. By subdividing the thickness at the two outer interfaces a simple approximation was obtained. Subsequently, he derived the equations of equilibrium, using the Euler's equations of stationary condition, indicated 
in the work of Hellinger [8] and more explicitly discussed by Hu [9], Washizu [10] and Reissner [11]. The incremental form of the Hellinger-Reissner complimentary functionals were used in the elastic-plastic analysis.

The small deflection elastic, large deflection and plastic analyses based on the conventional methods are too cumbersome and often a complete elastic-plastic analysis is extremely difficult. Even a complete elastic analysis obtained by solving an eighth order differential equation for various boundary conditions and type of loading is quite complex. Therefore, it is essential to employ numerical procedures, especially when large deflections are involved. The numerical methods of analysis are discussed in the next section.

\subsection{Numerical Methods of Analysis}

\subsubsection{Finite Difference Method}

The numerical procedures that can be employed to solve the governing differential equation of equilibrium include the finite difference method and the finite element method. Both methods discretize the continuum and both generate simultaneous algebraic equations for the nodal degrees of freedom. The finite difference method is well suited for the analysis of shells of revolution and also suited to 'pure' continua where there is just one medium, such as a homogeneous solid or a fluid [12]. However, for analyzing a structure that must be modeled by different forms e.g. a vehicle that combines a bar, beam, plate and shell components and for structures modeled by a mixture of materials, the finite difference method is too cumbersome. 


\subsubsection{Finite Element Method}

In the finite element method, the continuum is discretized into a finite number of elements or pieces. The nodal displacements are the unknown parameters. A function (or functions) is chosen to define uniquely the state of displacement within each finite element in terms of the nodal displacements. The strain-displacement relations define the unique state of strain within each element and hence, the state of stress throughout the element as well as on its boundaries. Finally, by assembling the finite elements to form the original structure, a stiffness matrix is formulated in terms of the unknowns. The nodal variables could then be solved in terms of the applied loads to complete the solution procedure.

A large number of finite elements with various geometrical shapes and displacement functions are available. A vast amount of literature exists on the application of finite element method to the analysis of thin shells [13]. Until recently, the majority of the work has been related to linear analysis. Such finite element formulations adopt the Kichhoff-Love assumptions to formulate the deep shell theories such as due to Koiter [14], Budiansky and Sanders [15]. Several finite elements have also been derived by modifying the continuum elements to comply with the shell-assumptions without adopting a rigorous shell theory. In case of fully non-linear analysis, additional problems arise in the formulation of finite elements due to various approximations used in reducing the complexity of non-linear theory.

The earliest finite element solutions for the linear elastic analysis of shells adopted assemblages of flat or facet elements. The rigid body modes were correctly represented and the technique gave results which were only surpassed by curved elements. Although the curved elements derived from Kichhoff-Love theory guarantee a high solution accuracy, they are complicated by convergence and compatibility requirements. 
Special elements have also been developed for the analysis of shallow shells. The semiloof element [16] was a result of an evolution process dating from the isoparametric element [17] and the membrane element [18]. Although the element seemed promising, difficulties later appeared due to the degeneration process, as the thickness of the shell was reduced. A great improvement of the model was achieved by a reduced-integration technique [19]. Martin and Owen [20] used the semiloof element with reduced-integration technique to carry out an elastic-plastic and geometrically non-linear analysis. Crisfield [13] used the degeneration concept and adopted a 8-noded rectangular element, with five degrees of freedom at the corner nodes and 2 in-plane degrees of freedom at the mid-side nodes, for the non-linear analysis of cylindrical panels. The formulation was extended to incorporate geometric and material non-linearities. Owen and Figueriras [21] used a mixed formulation of a 8 noded Serendipity element and a 9-noded heterosis element for the non-linear analysis of cylindrical shells.

Obviously, finite element methods have found extensive application in a vast range of engineering problems. However, for many structures having a regular geometric plan and simple boundary conditions, a full finite element analysis is often both extravagant and unnecessary [22]. In the non-linear analysis, quite often, it is necessary to use a very fine grid to obtain engineering accuracy. The cost of the solutions could be very high and sometimes impractical. To overcome these drawbacks in analyzing structures with a regular geometry and simple boundary conditions, the finite strip method was developed by Cheung [23].

\subsection{The Finite Strip Method}

The finite strip method is a generalized form of the finite element method which reduces the computational effort but still retains some of the versatility of the finite element 
method.

In this method, the structure is subdivided into strips or subdomains. The finite strip formulation, like its finite element counterpart, adopts a kinematic approach. However, unlike the finite element method, which uses polynomial displacement functions in all directions, the finite strip method employs simple polynomials in one direction and continuously differentiable functions in the other. The finite strip method also stipulates that these functions should satisfy a priori the displacement boundary conditions at the ends of the strip. The lateral displacement, $w$, of the finite strip, could, therefore, be represented by:

$$
w=\sum_{p=1}^{k} f_{p}(x) Y_{p}(y)
$$

The function $f_{p}(x)$ satisfies the displacement boundary conditions and approximates the deflected shape. $Y_{p}$ is a polynomial expression with undetermined constants.

The performance of the finite strip method in the non-linear analysis of plate structures was examined by Abayakoon et al [40] and found to be very satisfactory. Cheung extended the finite strip method from his initial analysis of flat plates [23] to analyze folded plate structures [22]. Later Cheung extended the theory to analyze cylindrical shells using a curved plate strip and subsequently, a shell strip [22]. Cylindrical shell strips for the vibration analysis of panels were also investigated by Cheung and found to be satisfactory. In the curved shell formulation, Cheung used linear interpolation polynomials for the in-plane displacements and cubic Hermitian polynomials to represent the normal displacement in the transverse direction. In the strip direction a continuously differentiable function was used to model the deflected shape and to satisfy the boundary conditions. Dawe [25] used a quintic polynomial distribution to represent the circumferential and the normal displacement components and a cubic polynomial distribution 
to represent the $u$ displacement component in the finite strip formulation, for the static analysis of diaphram supported cylindrical shells. To model mixed boundary conditions: concentrated loads and continuous spans, the spline finite strip method was developed by Cheung et al [26]. In this method each finite strip was divided into a number of subdomains. A set of spline functions was used to define the displacement variation inside each subdomain. The spline strip method was originally developed for the analysis of rectangular plates. Mizusawa [27] extended the application of the spline strip method to the vibration analysis of cylindrical shells. The prediction from this method seem to agree with the theoretical results. To date, the application of the finite strip method to the analysis of cylindrical shells has been mostly linear elastic. The effect of material and geometric non-linearities has not been incorporated. In the present work, a large deflection, elastic-plastic analysis of cylindrical shells has been conducted. The mathematical formulation of the problem is presented in the next chapter. 


\section{Chapter 3}

\section{Mathematical Formulation}

\subsection{Introduction}

Two formulations of the finite strip suitable for modelling cylindrical shells are developed. A flat plate strip element, shown in Fig. 3.1 , is used to approximate the cylindrical surface, and a more refined curved strip element, shown in Fig. 3.2, which models the curved cylindrical shell surface exactly, is also developed.

Section 3.2 introduces the finite strip discretization of the circular cylindrical shell. The displacement components involved in both the Flat Plate and the Curved Shell element are discussed. The basis for the selection of displacement functions and their choice depending on the boundary conditions and the finite element theory is presented in Section 3.3. Section 3.4 presents the strain-displacement relations employed in the present analysis with a special mention to the differences between the two finite strip models and the consequent implications on the choice of shape functions for the displacement components.

The constitutive relations are presented in Section 3.5. The formulation of the stiffness matrix using the virtual work principle is presented in Section 3.6. Since the scope of the present formulation includes non-linear effects - both geometric and material - the resulting equations are non-linear. The Newton-Raphson iterative procedure is employed for the solution of the non-linear equations. The mathematical derivation of 
the Newton-Raphson procedure is presented in Section 3.7. Some comments on the numerical integration used are made in Section 3.8. Important aspects of the computer implementation of the finite strip formulation are presented in Section 3.9.

\subsection{Finite Strip Discretization}

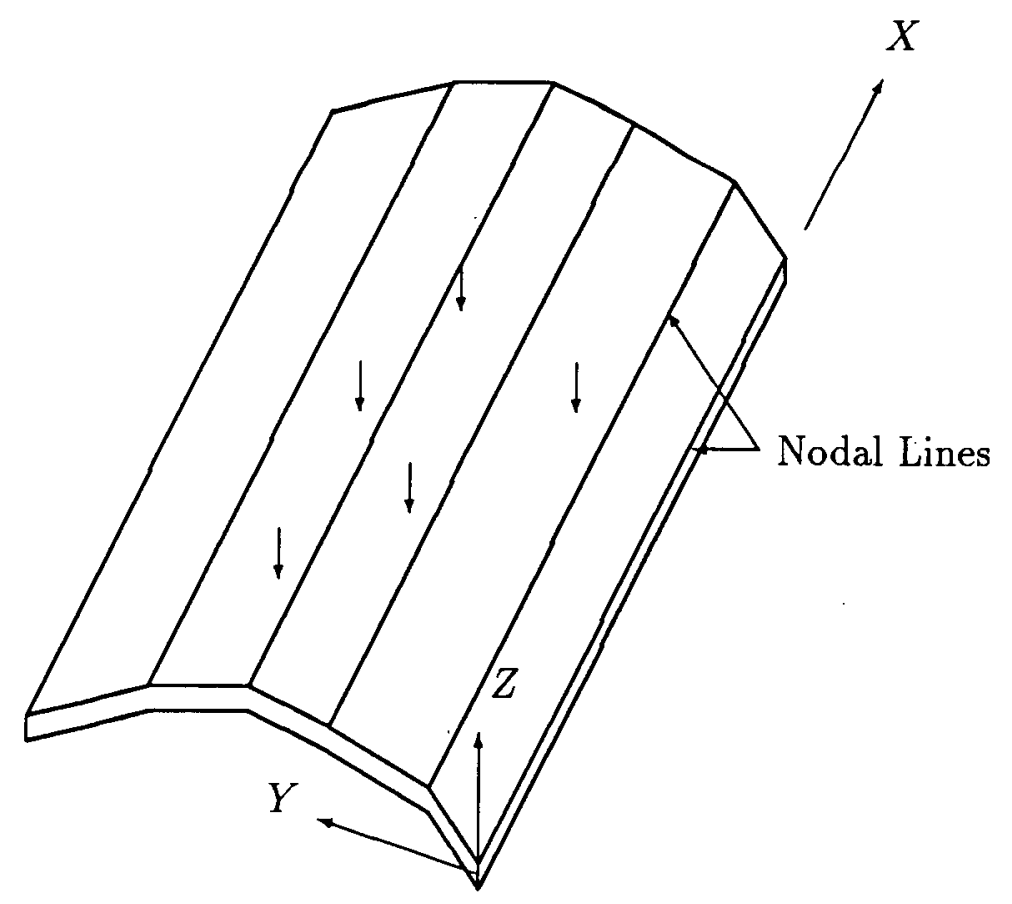

Figure 3.1: Flat Plate Discretization

\subsubsection{Flat Plate Model}

The finite strip discretization of a circular cylindrical shell is shown in Fig. 3.1. The global co-ordinate system $X Y Z$ is rectangular cartesian. The finite strips are connected along common nodal lines. The inter-element compatibility is satisfied by maintaining the continuity of the displacement variables. Although there are no restrictions on the geometry of the flat plate element in terms of its thickness or width, they will be assumed 
to be constant in this formulation. The arrows shown in Fig. 3.1 represent the lateral loading on the shell. In this formulation the loading pattern on the shell is taken as either a uniform pressure load or the self-weight of the shell.

\subsubsection{Curved Shell Model}

Fig. 3.2 shows the finite strip discretization using curved shell element. In this model the global co-ordinate system $X Y Z$ is defined in polar co-ordinates. The global $X$-axis, located at the mid-surface, is along the longitudinal or the non-curved direction of the shell while the global $Y$ and $Z$ axes of the co-ordinate system are along the transverse and the radial directions of the cylindrical shell respectively. Therefore, for a circular cylindrical shell of a constant radius of curvature $R$, the global $Z$-co-ordinate at each point on the middle surface of the shell will be zero. The circumferential co-ordinate of any point will vary as a function of the angle $\beta$ subtended by the shell element at the center of curvature.

Once again, the inter-element compatibility has to be satisfied along the nodal line, where the two curved shell elements are connected, by matching the displacement variables along these lines. The loading on the shell, represented by arrows in Fig. 3.2, represents the the lateral loading. The loading pattern in the shell is assumed to be uniform in this formulation. A uniform pressure load or a self-weight analysis can be carried out with relative ease.

\subsubsection{Nodal-Line Variables in Finite Strip Models}

The mathematical formulation used in the analysis of cylindrical shells assumes that the variables defined along each nodal line adequately represent the displacement components in the real structure. As mentioned in the preceding chapter, the membrane or in-plane 


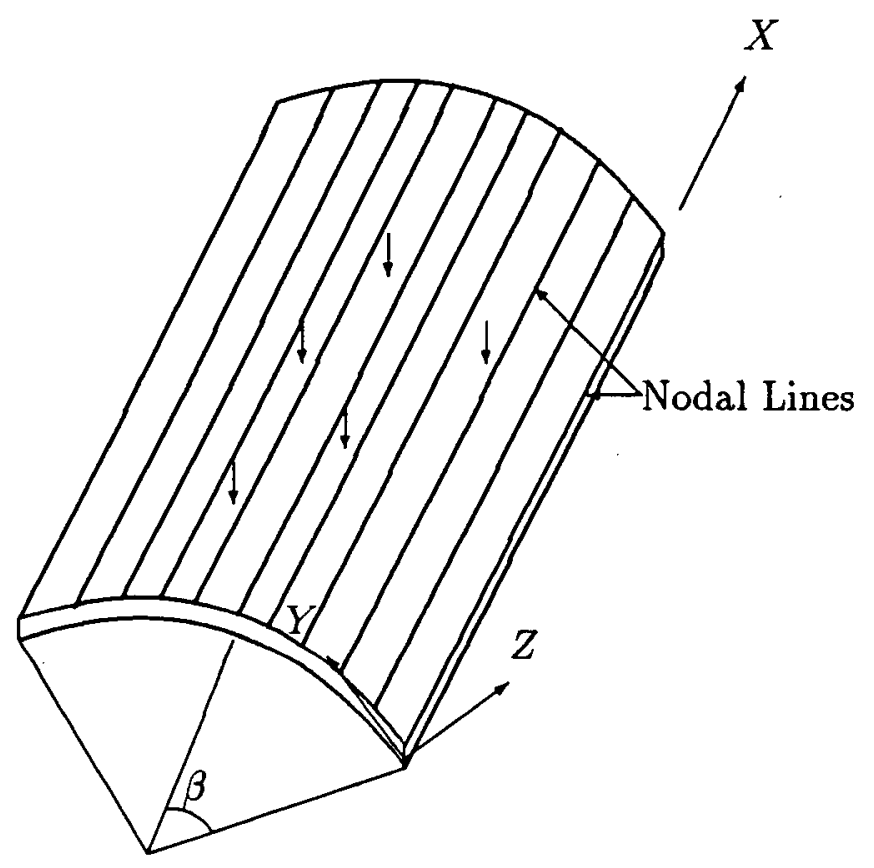

Figure 3.2: Curved Shell Discretization

displacements are significant as compared with the bending displacements in a cylindrical shell. Therefore, it is essential to include both in-plane and out-of-plane displacements as variables along each nodal line, even for small deflection theory.

Fig. 3.3 shows an isolated flat plate element with a local co-ordinate system $x y z$. Fig. 3.4 and Fig. 3.5 show two types of curved shell elements, denoted as curved shell element I and curved shell element II.

The variables along each nodal line in all the three cases are defined by the maximum modal amplitude of the longitudinal displacement functions for each of the displacement components $u, v$ and $w$. The flat plate element and the curved shell element I, shown in Fig. 3.3 and Fig. 3.4 respectively, have two nodal lines 1 and 2. Along each nodal line, the displacement components are represented by two in-plane displacements $u$ and $v$, an out-of-plane displacement $w$ and a rotation $\theta=\frac{\partial w}{\partial y}$. Hence, each finite strip has 8 variables in this formulation. 


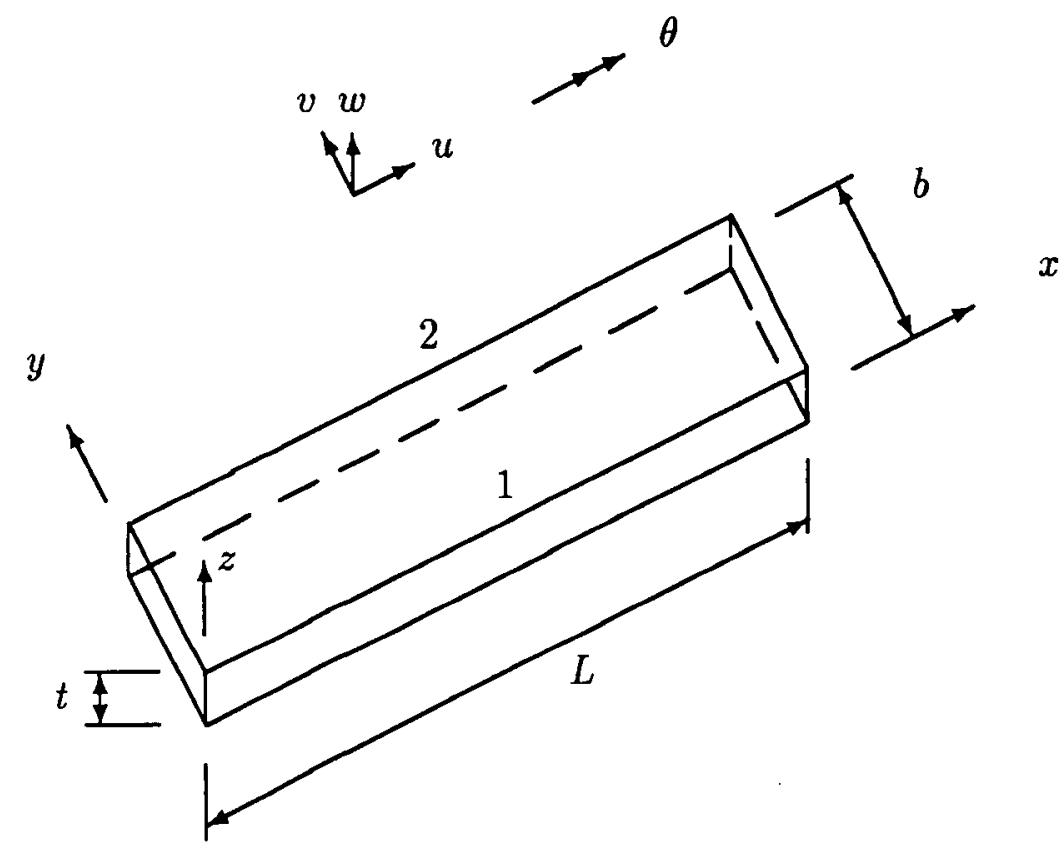

Figure 3.3: A Flat Plate Element

The curved shell formulation in Fig. 3.5 has three nodal lines - two external and one internal - in each element. In addition to the two external nodal lines there exists an internal nodal line where two displacement variables $u$ and $v$ are defined. Hence, in this formulation, each curved shell finite strip element has 10 variables.

\subsection{Displacement Functions}

\subsubsection{General}

The choice of shape functions for the displacement components of the finite strip in the analysis of cylindrical shells assumes considerable significance. The deflection profile needs to be adequately modelled, the displacement boundary conditions need to be satisfied exactly and the criteria for convergence needs to be fulfilled. 


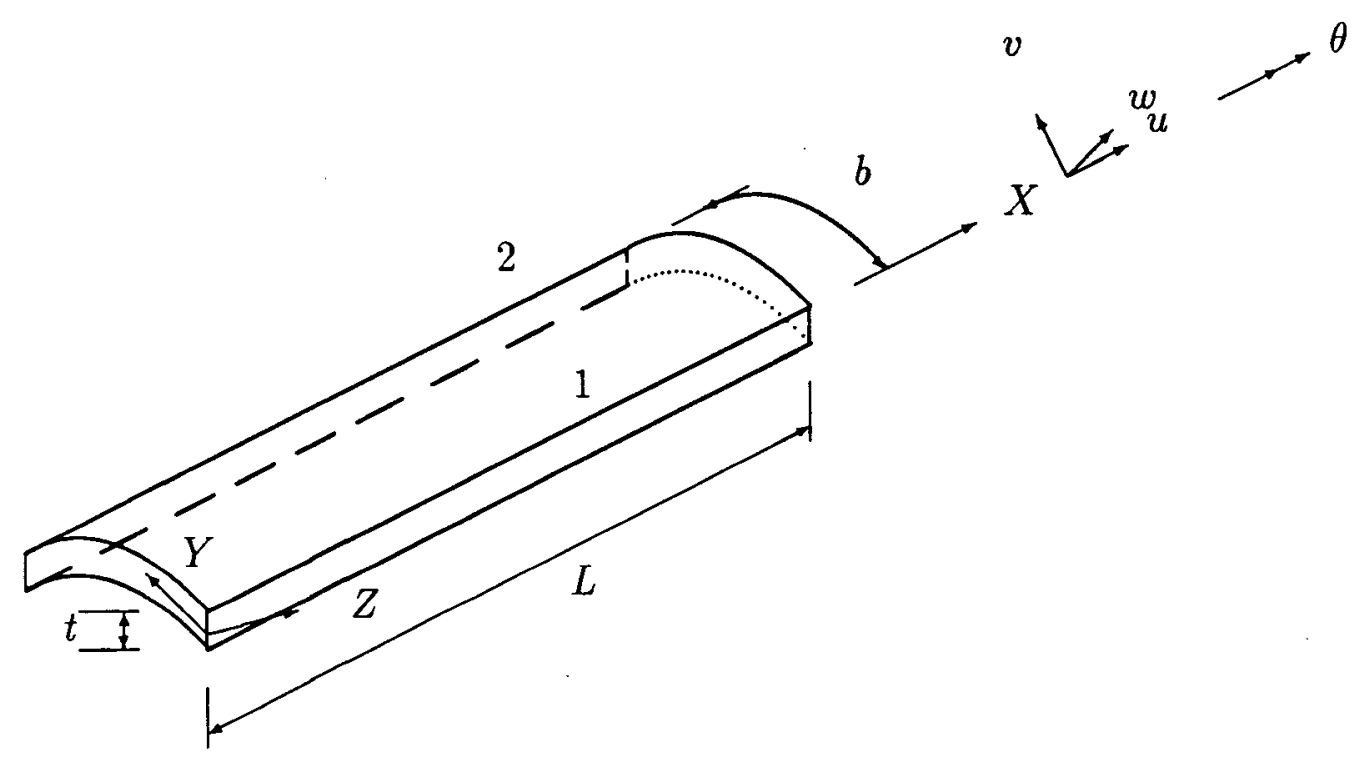

Figure 3.4: Curved Shell Element I

Bearing these considerations in mind, let us consider the logical options for representing the displacement components in the longitudinal direction. The displacement functions in the longitudinal direction have to satisfy the boundary conditions at the transverse ends. In the present study, two types of support conditions are examined simply supported and clamped. The boundary conditions along the straight edges of the cylindrical shell could be simply supported, clamped or free.

The displacement functions for the displacement components $u, v$ and $w$ are defined as: 


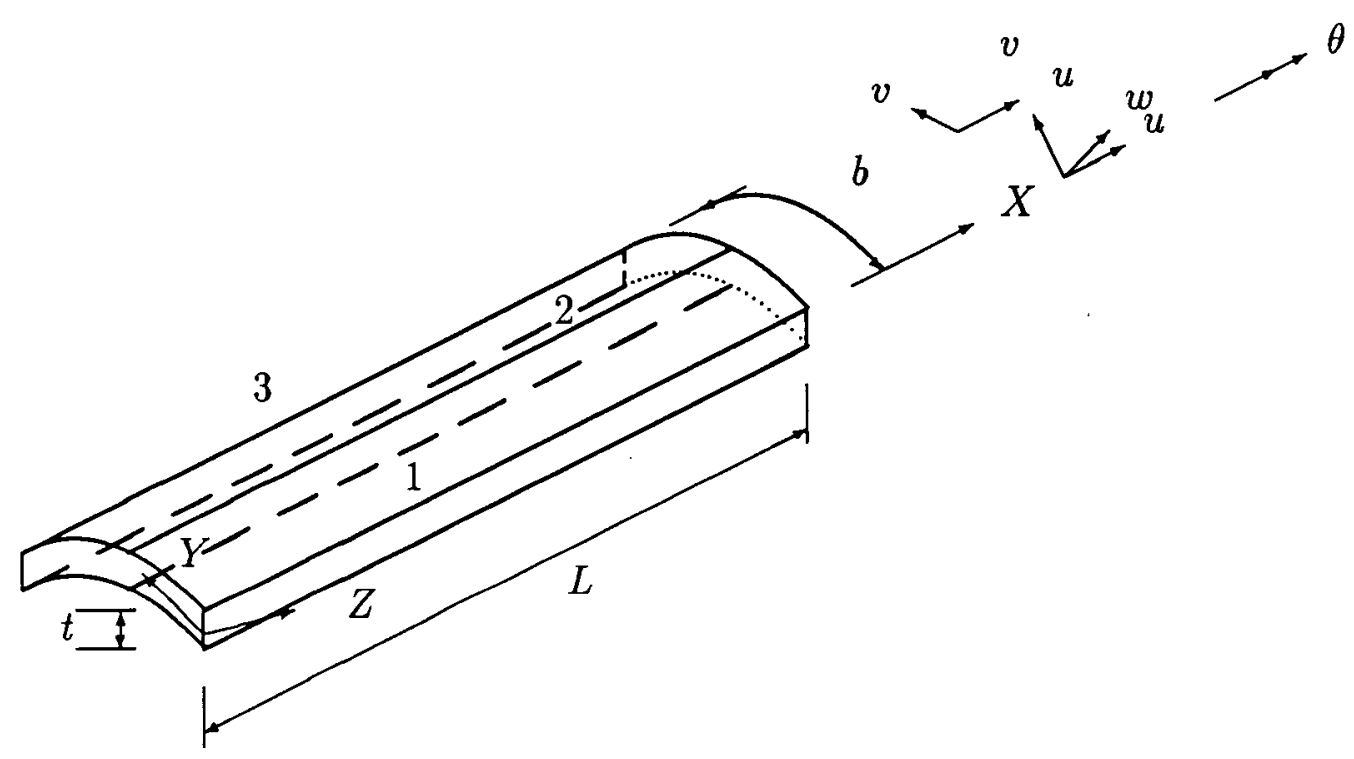

Figure 3.5: Curved Shell Element II

$$
\begin{aligned}
& u=g_{m}^{u}(\xi) Y_{m}(\eta) \\
& v=g_{n}^{v}(\xi) Y_{n}(\eta) \\
& w=g_{p}^{w}(\xi) Y_{p}(\eta)
\end{aligned}
$$

where $\xi=\frac{x}{L}$ and $\eta=\frac{y}{R}$.

In the longitudinal direction, the variation of the displacement components is defined by the functions $g(\xi)$. In the $y$-direction, the variation is represented by polynomials, $Y_{p}$, as in the finite element approach. The displacement functions in the transverse direction must satisfy all requirements for convergence according to finite element theory:

1. The displacement field within the element must be continuous. 
2. The element must be able to assume a state of constant strain.

3. All rigid body modes must be present.

4. Inter-element compatibility must be satisfied.

\subsubsection{Flat Plate Strips}

The displacement functions in the $x$ direction i.e. longitudinal direction for the most common displacement boundary conditions are given below:

(a) Simply Supported ends

1. No axial constraint

$$
\begin{array}{cc}
g_{m}^{u}(\xi)=\cos m \pi \xi ; & m=1,3,5, \cdots, \\
g_{n}^{v}(\xi)=\sin n \pi \xi ; & n=1,3,5, \cdots, \\
g_{p}^{w}(\xi)=\sin p \pi \xi ; & p=1,3,5, \cdots,
\end{array}
$$

2. Constrained in the axial direction

$$
g_{m}^{u}(\xi)=\sin m \pi \xi ; \quad m=2,4,6, \cdots
$$

(b) Clamped ends

The shape functions for the $u$ displacement remain the same i.e. $g_{m}^{u}$, as in the simply supported case above.

At a common nodal line adjacent flat plate strips are rotated with respect to one another and this leads to an interaction of $v$ and $w$ displacements. In order to satisfy compatibility between adjacent finite strips, the variation of $v$ and $w$ displacements in 
the longitudinal direction must be the same. The longitudinal modes chosen to represent $w$ displacement are the clamped beam vibration modes, $\phi_{p}(\xi)$. Therefore, $g_{n}^{v}(\xi)$, i.e. the variation of the $v$ displacement must also be represented by $\phi_{n}(\xi)$. Then, the displacement functions chosen to represent the tangential and the normal displacement components, $v$ and $w$ respectively, of the clamped cylindrical shell, could be written as:

$$
\begin{aligned}
g_{n}^{v}(\xi) & =\phi(\xi) \quad n=1,3,5, \cdots \\
g_{p}^{w}(\xi) & =\phi(\xi) \\
& =\frac{\left[\alpha_{p}\left(\sinh \beta_{p} \xi-\sin \beta_{p} \xi\right)+\left(\cosh \beta_{p} \xi-\cos \beta_{p} \xi\right)\right]}{A_{p}} ; \quad p=1,3,5, \cdots,
\end{aligned}
$$

where,

$$
\begin{aligned}
& A_{p}=\alpha_{p}\left(\sinh 0.5 \beta_{p} \xi-\sin 0.5 \beta_{p} \xi\right)+\left(\cosh 0.5 \beta_{p} \xi-\cos 0.5 \beta_{p} \xi\right) \\
& \alpha_{p}=\frac{\cos \beta_{p}-\cosh \beta_{p}}{\sinh \beta_{p}-\sin \beta_{p}}
\end{aligned}
$$

and $\beta_{p}$ are the solutions of the transcedental equation $\cosh \beta_{p}=\cos \beta_{p}$. These $\phi(\xi)$ are the clamped beam vibration modes.

The displacement functions in the transverse direction are chosen to be linear for in-plane displacements $u$ and $v$, and cubic shape functions for the normal or the $w$ displacement. These are the well known Hermitian polynomials as used in the finite element analysis of beam bending. These functions were used for the finite strip analysis of stiffened plates by Abayakoon [24].

The $u, v$ and $w$ displacement distributions of a single strip can be written in terms of nodal displacements by combining the shape functions. For a strip of size $L \times b$ : 


$$
\begin{aligned}
u= & {\left[(1-\eta) u_{1 m}+\eta u_{2 m}\right] g_{m}^{u}(\xi) } \\
v= & {\left[(1-\eta) v_{1 n}+\eta v_{2 n}\right] g_{n}^{v}(\xi) } \\
\text { and } & \\
w= & {\left[\left(1-3 \eta^{2}+2 \eta^{3}\right) w_{1 p}+\left(\eta-2 \eta^{2}+\eta^{3}\right) b \theta_{1 p}+\right.} \\
& \left.\left(3 \eta^{2}-2 \eta^{3}\right) w_{2 p}+\left(\eta^{3}-\eta 2\right) b \theta_{2 p}\right] g_{p}^{w}(\xi)
\end{aligned}
$$

where $\xi=\frac{x}{L}$ and $\eta=\frac{y}{b}$

$u_{1 m}, v_{1 n}, w_{1 p}$ etc are the nodal-line variables and the summation convention is used for the repeated indices $m, n,, p$.

\subsubsection{Curved Shell Strip I}

The curved shell strip in Fig. 3.4 resembles the plate strip in that the total number of nodal-line variables in a finite strip are identical. The shape functions along both directions - longitudinal and transverse will be the same as used in the flat plate finite strip formulation. One of the major objectives of using Curved Shell strips I with linear interpolation polynomials for the in-plane displacement components was to keep the total number of degrees of freedom in every finite strip to be the same as in the flat plate formulation. Although this might yield a variable rate of convergence as discussed in Section 3.4.3, nevertheless, the possibility of better accuracy, by incorporating the exact strain-displacement relations for the cylindrical shell, was investigated.

\subsubsection{Curved Shell Strip II}

The curved shell strip in Fig. 3.5 is a modification of the flat plate and the curved shell strip I. In addition to the two external nodal lines, an internal nodal line is introduced at the middle of the finite strip. The displacement variables represented by this nodal 
line are the in-plane displacements $u$ and $v$.

The displacement functions in the longitudinal direction will remain the same as in the other two formulations. However, with the introduction of an internal nodal line the variation in the transverse direction changes. The variation of the in-plane displacements $u$ and $v$ will be quadratic rather than linear. For a curved shell strip of size $L \times b$, the displacement components can now be represented as:

$$
\begin{aligned}
u= & {\left[\left(1-3 \eta+2 \eta^{2}\right) u_{1 m}+\left(4 \eta-4 \eta^{2}\right) u_{2 m}+\left(-\eta+2 \eta^{2}\right) u_{3 m}\right] g_{m}^{u}(\xi) } \\
v= & {\left[\left(1-3 \eta+2 \eta^{2}\right) v_{1 n}+\left(4 \eta-4 \eta^{2}\right) v_{2 n}+\left(-\eta+2 \eta^{2}\right) v_{3 n}\right] g_{n}^{v}(\xi) } \\
w= & {\left[\left(1-3 \eta^{2}+2 \eta^{3}\right) w_{1 p}+\left(\eta-2 \eta^{2}+\eta^{3}\right) b \theta_{1 p}+\right.} \\
& \left.\left(3 \eta^{2}-2 \eta^{3}\right) w_{2 p}+\left(\eta^{3}-\eta 2\right) b \theta_{2 p}\right] g_{F}^{w}(\xi)
\end{aligned}
$$

\subsection{Strain Displacement Relations}

\subsubsection{Flat Plate - Bending Theory}

The well known large deflection strain displacement relations for plate bending are [4]:

$$
\begin{array}{r}
\epsilon_{x}=\frac{\partial u}{\partial x}-z \frac{\partial^{2} w}{\partial x^{2}}+\frac{1}{2}\left(\frac{\partial w}{\partial x}\right)^{2}+\frac{1}{2}\left(\frac{\partial v}{\partial x}\right)^{2} \\
\epsilon_{y}=\frac{\partial v}{\partial y}-z \frac{\partial^{2} w}{\partial y^{2}}+\frac{1}{2}\left(\frac{\partial w}{\partial y}\right)^{2}+\frac{1}{2}\left(\frac{\partial v}{\partial y}\right)^{2} \\
\gamma_{x y}=2 \epsilon_{x y}=\frac{\partial u}{\partial y}+\frac{\partial v}{\partial x}-2 z \frac{\partial^{2} w}{\partial y \partial x}+\frac{\partial w}{\partial x} \frac{\partial w}{\partial y}+\frac{\partial v}{\partial x} \frac{\partial v}{\partial y}
\end{array}
$$

where $\epsilon_{x}, \epsilon_{y}$ and $\epsilon_{x y}$ are the non-zero components of strain. $\gamma_{x y}$ is termed the engineering shear strain. $u, v$ and $w$ are the displacement at the mid-surface of the plate in the $x, y$ and $z$ directions respectively. The mid-surface coincides with the $x y$ plane and $z$ is 
measured perpendicular to this surface.

The bending component of the strain is represented by the terms varying linearly in $z$; the other components of the strain occur due to stretching of the middle surface. The nonlinear terms due to the displacement component $u$, have been neglected as they are small as compared with the $w$-displacement. Since the main philosophy in this formulation is to model cylindrical shells by flat elements, the strips are oriented at varying angles leading to an interaction of the $v$ and $w$ displacements. Therefore, the non-linear terms due to displacement component $v$ have been retained in the strain displacement relations.

\subsubsection{Cylindrical shell - bending theory}

The strain displacement relations for the bending of a cylindrical shell including large displacement effects are [4]:

$$
\begin{array}{r}
\epsilon_{\boldsymbol{x}}=\frac{\partial u}{\partial x}-z \frac{\partial^{2} w}{\partial x^{2}}+\frac{1}{2}\left(\frac{\partial w}{\partial x}\right)^{2} \\
\epsilon_{y}=\frac{\partial v}{\partial y}+\frac{w}{R}-z\left(\frac{\partial^{2} w}{\partial y^{2}}-\frac{1}{R} \frac{\partial v}{\partial y}\right)+\frac{1}{2}\left(\frac{\partial w}{\partial y}\right)^{2} \\
\gamma_{x y}=2 \epsilon_{x y}=\frac{\partial u}{\partial y}+\frac{\partial v}{\partial x}-z\left(2 \frac{\partial^{2} w}{\partial y \partial x}-\frac{1}{R} \frac{\partial v}{\partial x}\right)+\frac{\partial w}{\partial x} \frac{\partial w}{\partial y}
\end{array}
$$

Here $\epsilon_{x}, \epsilon_{y}$ and $\epsilon_{x y}$ are referred to the polar co-ordinate system and $R$ is the radius of curvature. Once again, the bending components of the strain vary linearly with $z$, which is the radial distance from the middle surface of the shell. Compared with the flat plate equations, if we examine the tangential component of the strain, we observe the $\left(\frac{w}{R}\right)$ term which represents the stretching of the middle surface due to a radial displacement $w$. The non-linear terms due to $u$ and $v$ displacements, being relatively smaller than the $w$ displacements, are neglected. These assumptions of retaining the non-linear terms due 
to $w$ while neglecting the terms due to in-plane displacements $u$ and $v$ are consistent with Donell's [44] approximations in the derivation of a simplified non-linear shell theory.

\subsubsection{Order of Accuracy Analysis}

As mentioned in Section 3.3, the choice of shape functions for the various formulations is dependent on the strain displacement relations. The criteria for finite element convergence has to be satisfied. Given the strain-displacement relations and the criteria for convergence, it is possible to predict the order of strain-energy convergence as the finite strip grid is refined.

Let us examine the terms in the strain energy expression for a cylindrical shell for a better appreciation of the choice of shape functions in the tangential direction for the two Curved Shell formulations. The expression for the strain energy of a cylindrical shell [32] for the linear case in polar co-ordinates, is given by:

$$
\begin{aligned}
U= & \frac{D}{2} \int_{0}^{b} \int_{0}^{a}\left\{w_{x x}^{2}+w_{y y}^{2}+2 \nu w_{x x} w_{y y}+2(1-\nu) w_{x y}^{2}\right. \\
& +\frac{12}{t^{2}}\left[u_{x}^{2}+v_{y}^{2}+2 \nu u_{x} v_{y}+\frac{1-\nu}{2}\left(u_{y}+v_{x}\right)^{2}\right] \\
& +\frac{1}{R^{2}}\left[v_{y}^{2}++2(1-\nu) v_{x}^{2}\right] \\
& +\frac{1}{R}\left[\frac{24}{h^{2}}\left(v_{y}+\nu u_{x}\right) w-2 v_{y}\left(w_{y y}+\nu w_{x x}\right)\right] \\
& \left.-4(1-\nu) w_{x y} v_{x}\right\} d x d y
\end{aligned}
$$

where $D=\frac{E t^{3}}{12\left(1-\nu^{2}\right)}$ is the rigidity modulus, $\nu$ is the Poisson's ratio $R$ is the radius of curvature and $t$ is the thickness of the shell and the subscripts denote derivatives. 
The terms in the strain energy expression involving derivatives with respect to $x$ need not be examined as the shape functions in the longitudinal direction in all formulations remain the same and are sufficiently differentiable. Let us examine the terms in the tangential direction in the two Curved Shell formulations:

1. Curved Shell I

(a) $v_{y}^{2}:$ Since $v$ is linear in the tangential direction

$$
\begin{gathered}
\text { Taylor series error in } v_{y}=\mathrm{O}(l) \\
\text { Strain Energy error }=\mathrm{O}\left(l^{2}\right)
\end{gathered}
$$

(b) $u_{y}^{2}:$ Since $u$ is linear in the tangential direction,

$$
\begin{gathered}
\text { Taylor series error in } u_{y}=O(l) \\
\text { Strain Energy error }=O\left(l^{2}\right) .
\end{gathered}
$$

(c) $w_{y y}^{2}$ : Since $w$ is cubic in the tangential direction,

$$
\begin{gathered}
\text { Taylor series error in } w_{y y}=O\left(l^{2}\right) \\
\text { Strain Energy Error }=O\left(l^{4}\right)
\end{gathered}
$$

where $l$ is the width of the element in the tangential direction.

The minimum order of convergence of strain energy varies from quadratic for membrane components to fourth order for bending and is, therefore, not consistent.

2. Curved Shell II

(a) $v_{y}^{2}:$ Since $v$ is quadratic in the tangential direcion,

Taylor series error in $v_{y}=\mathrm{O}\left(l^{2}\right)$

$$
\text { Strain Energy error }=O\left(l^{4}\right)
$$


(b) $u_{y}^{2}:$ Since $u$ is quadratic in the tangential direction,

$$
\begin{gathered}
\text { Taylor series error in } u_{y}=O\left(l^{2}\right) \\
\text { Strain Energy error }=O\left(l^{4}\right)
\end{gathered}
$$

(c) $w_{y y}^{2}$ : Since $w$ is cubic in the tangential direction,

$$
\begin{gathered}
\text { Taylor series error in } w_{y y}=O\left(l^{2}\right) \\
\text { Strain Energy Error }=O\left(l^{4}\right)
\end{gathered}
$$

where $l$ is the width of the element in the tangential direction.

Therefore, from considerations of finite element formulation, a consistent order of convergence is expected in this formulation by assuming a quadratic variation of the in-plane displacements in the tangential direction.

\subsection{Constitutive Relations}

The assumed stress-strain relationship for the shell material under uniaxial loading is represented in Fig. 3.6

$\sigma_{\circ}$ and $\epsilon_{\circ}$ are the uniaxial yield stress and the uniaxial strain respectively. $E$ and $E_{T}$ are the slopes of this bilinear representation. In carrying ont an elastic-plastic analysis of a given structure, three major aspects of the material behavior need to be specified. One needs to know when the first yield occurs, what is the governing flow rule i.e. behavior of the material after yielding has taken place and finally, how does the yield criteria change when plastic flow occurs. This is summarized below:

1. The shell material remains elastic till the stresses exceed a pre-defined value of stress called the yield stress. The combination of multiaxial stresses which contribute to the yield stress is determined by the choice of a suitable yield criterion. 


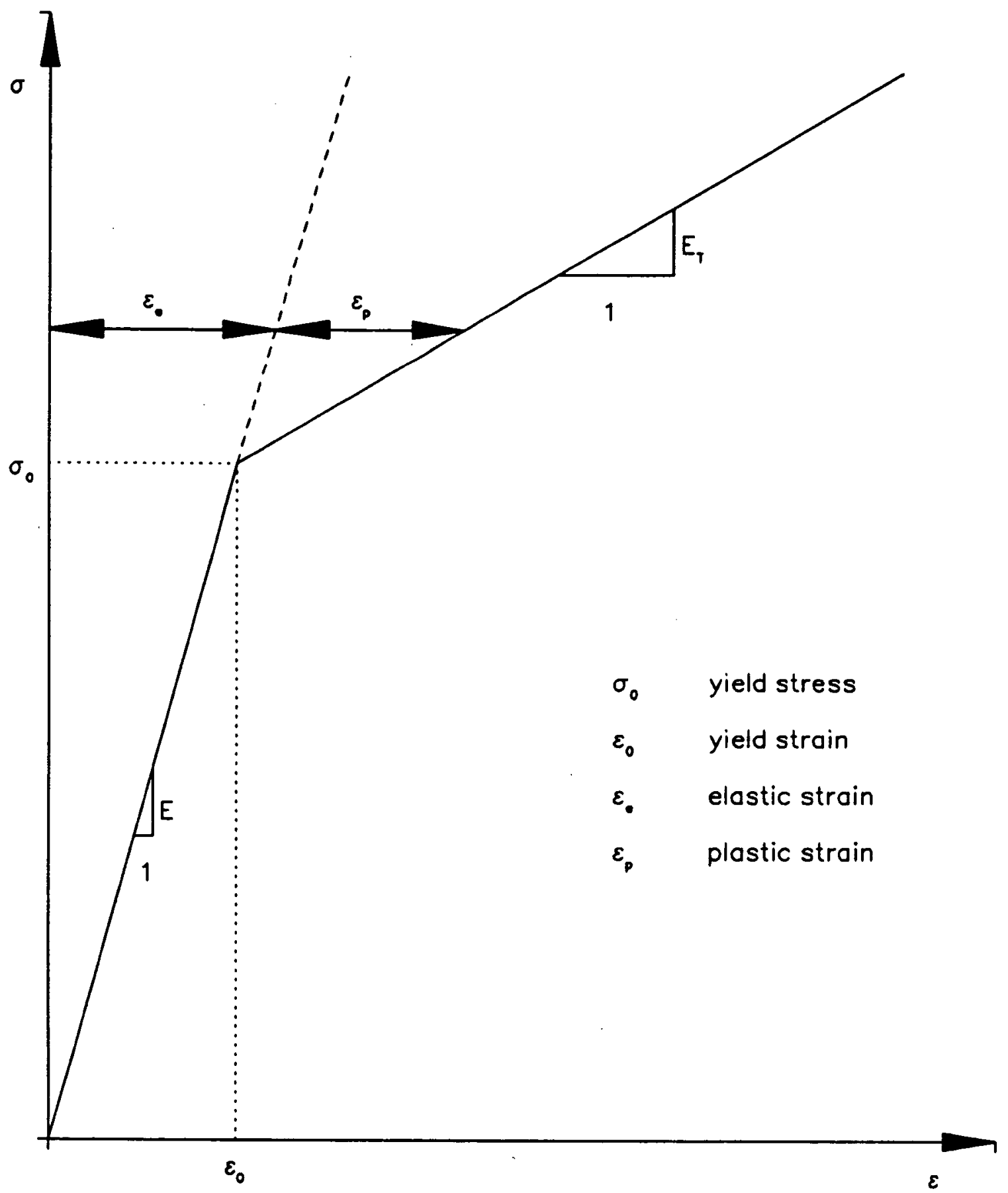

Figure 3.6: Stress-Strain relationship 
2. Once the shell material has yielded, the behavior of the structure i.e. the direction of strain-increments is determined by flow rules which need to be defined.

3. The yield criteria of the shell material varies with the plastic flow. To define this variation, a suitable hardening rule needs to be chosen.

The yield of ductile metals has been successfully predicted by the von-Mises criterion. The von-Mises criterion is based on the assumption that yielding occurs when the distortion or the shear strain energy equals the distortion strain energy at yield in simple tension.

The distortion energy $U_{d}$ is written as:

$$
U_{d}=\frac{1}{2 G} J_{2}
$$

where $\mathrm{G}$ is the shear modulus of the material and

$$
J_{2}=\frac{1}{6}\left[\left(\sigma_{1}-\sigma_{2}\right)^{2}+\left(\sigma_{2}-\sigma_{3}\right)^{2}+\left(\sigma_{3}-\sigma_{1}\right)^{2}\right]
$$

is the second invariant of the stresses.

At the yield point in simple tension

$$
\sigma_{1}=\sigma_{o}, \text { and } \sigma_{2}=\sigma_{3}=0
$$

Therefore,

$$
J_{2}=\frac{1}{3} \sigma_{\circ}^{2}
$$

Hence, the yield condition, as per von-Mises theory is given theory is given by

$$
\frac{1}{2}\left[\left(\sigma_{1}-\sigma_{2}\right)^{2}+\left(\sigma_{2}-\sigma_{3}\right)^{2}+\left(\sigma_{3}-\sigma_{1}\right)^{2}\right]=\sigma_{o}^{2}
$$


where $\sigma_{1}, \sigma_{2}$ and $\sigma_{3}$ are the principal components of the three dimensional stress.

The behavior of the material after yielding is governed by the plastic stress-strain relationships. The generalized stress-strain relations to include both elastic and plastic components of strain is due to Prandtl and Reuss which correspond to an associated flow rule using the von-Mises yield function [28]. In this formulation, an associated flow rule is assumed.

At the time of first yield, the yield surface is defined by $K$ which represents the initial yield surface. If the material is perfectly plastic, plastic flow occurs and the yield surface remains fixed. However, for a material which strain hardens, the yield surface must change as further plastic loading occurs. This is referred to as strain hardening. In isotropic hardening, the yield surface will expand with stress and strain history but will retain the same initial shape as shown in Fig. 3.7 (b).

The assumption of isotropic hardening is simple to handle mathematically but it does not account for the Bauschinger effect. The implication of the Bauschinger effect is that the initial shape of the yield surface, or the symmetry with respect to the origin in the stress space, is not retained in subsequent plastic deformations. The Bauschinger effect wrould tend to reduce the size of the locus on one side as that on the other side is increased.

To incorporate the Bauschinger effect, a kinematic hardening model is introduced. The initial yield surface translates without deforming while maintaining the total elastic range constant. This model accounts for the Bauschinger effect but somewhat overcorrects it by maintaining the total elastic range constant. In the present study, the elastic-plastic response of cylindrical shells is investigated only for the case of monotonically increasing load and the Bauschinger effect is not important. Therefore, an isotropic hardening model will be used in this formulation.

The finite strip formulation of the elastic-plastic constitutive relations is described below: 


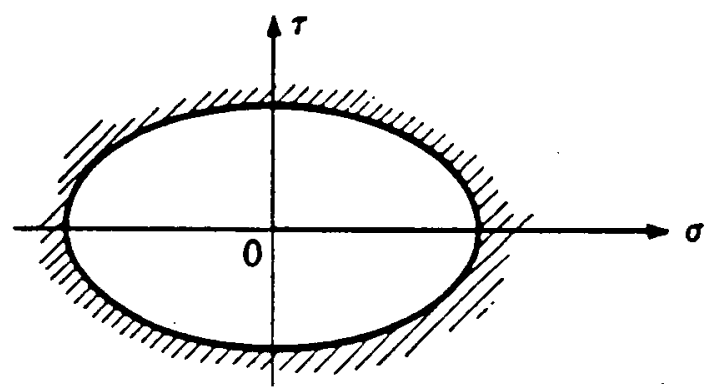

(a) Perfectly plastic

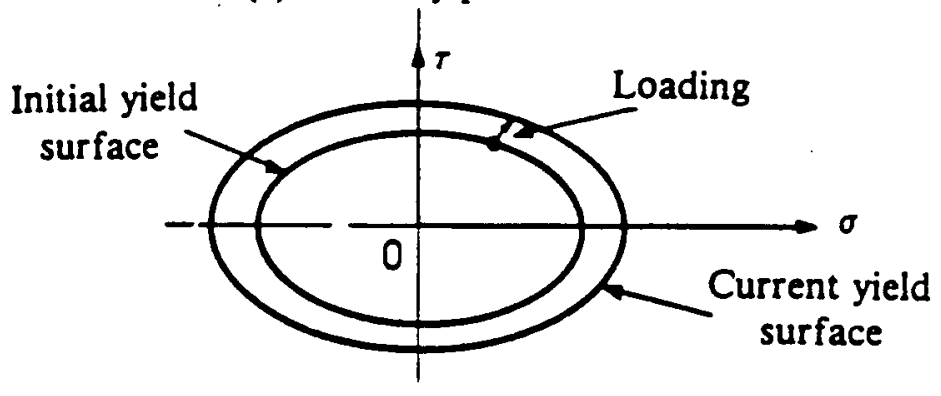

(b) Isotropic strain hardening

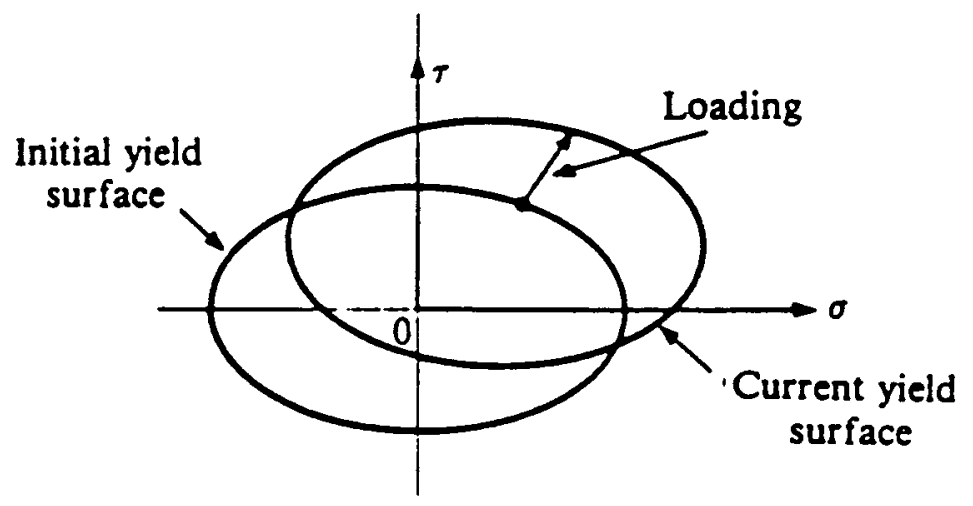

(c) Kinematic strain hardening

Figure 3.7: Hardening Models 


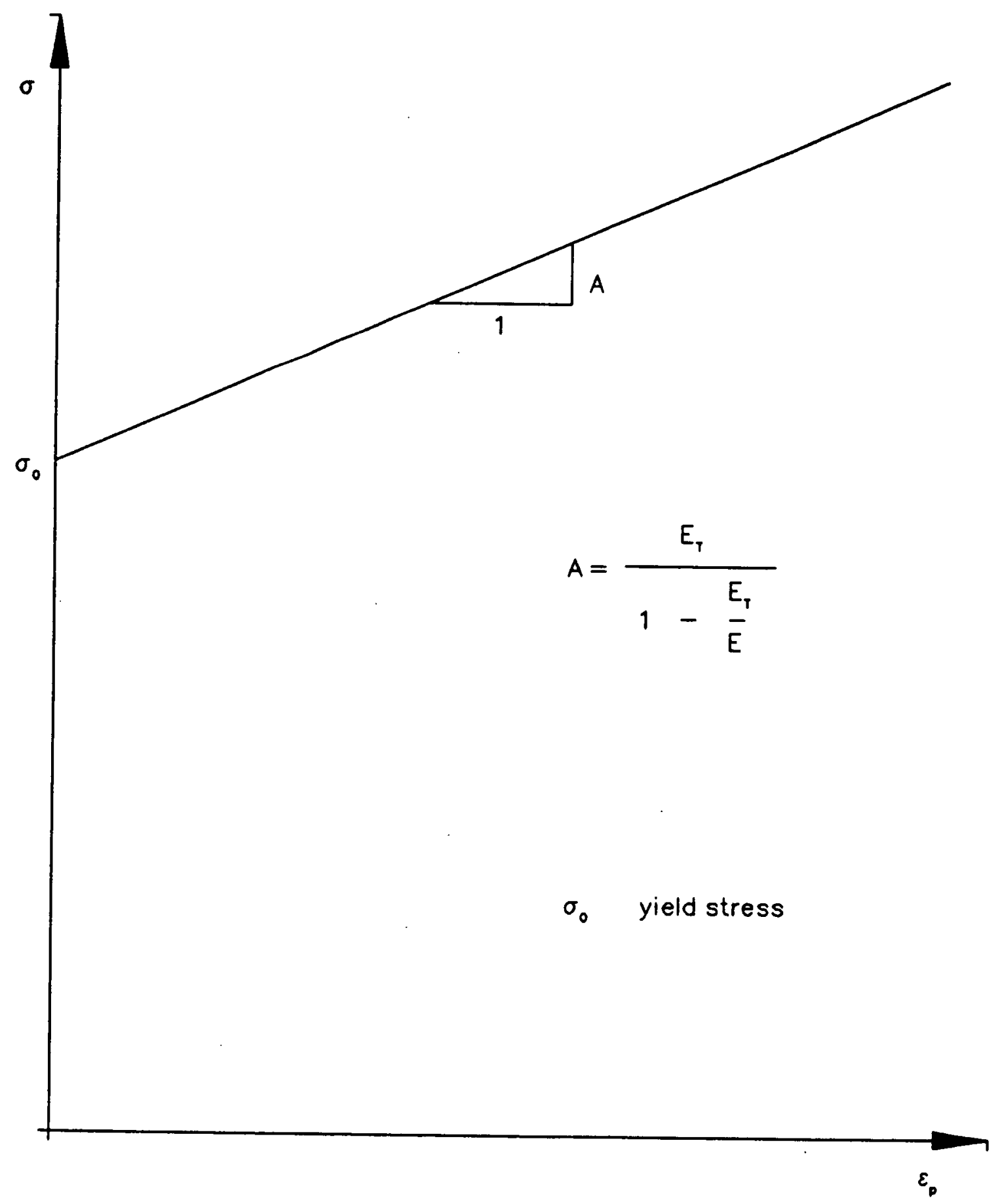

Figure 3.8: Stress-plastic strain Relationship 


$$
\{d \sigma\}=\left[D_{T}\right]\{d \epsilon\}
$$

where,

$$
\left[D_{T}\right]=[D]-[D]\{V\}\{V\}^{T}[D]\left[A+\{V\}^{T}[D]\{V\}\right]^{-1}
$$

and,

$$
\{d \epsilon\}=\left\{\begin{array}{c}
d \epsilon_{x} \\
d \epsilon_{x} \\
d \epsilon_{x}
\end{array}\right\}
$$

In Eq. ( 3.14 ) $[D]$ is the elasticity matrix, $\{V\}$ is a vector defined by the derivative of the yield function $\{V\}=\left\{\frac{\partial F}{\partial\{\sigma\}}\right\}$ and $A$ is the slope of the stress-plastic strain relationship in a uniaxial test. Fig. 3.8 shows the stress-plastic strain relationship for the assumed bilinear variation shown in Fig. 3.6. Then,

$$
A=\frac{E_{T}}{1-\frac{E_{T}}{E}}
$$

The elasto-plastic matrix replaces the elasticity matrix in incremental analysis. The finite strip analysis gives the stress increment for any given strain increment. The stress vector $\{\sigma\}$ is then computed at the end of the current iteration. This stress vector is subsequently used to calculate an equivalent effective stress $\bar{\sigma}$ given by:

$$
\bar{\sigma}^{2}=\sigma_{x}^{2}-\sigma_{x} \sigma_{y}+\sigma_{y}^{2}+3 \tau_{x y}^{2}
$$

This effective stress $\bar{\sigma}$, is the two-dimensional representation of $J_{2}$ given in Eq.( 3.11 ). Initial yield takes place when $\bar{\sigma}$ exceeds $\sigma_{\circ}$ for the first time, where $\sigma_{\circ}$ is the yield stress in uniaxial loading. As soon as the first yield occurs, the stresses are scaled down to 
coincide with the yield surface and plastic constitutive relations are employed from that point. The unloading or loading of the material is dependent on whether $\bar{\sigma}$ has beein decreased or increased. If unloading takes place, the iterative procedure is repeated with the elastic constitutive relations until yielding occurs again. If loading occurs while in the plastic region, the iteration is repeated with plastic constitutive relations.

\subsection{Stiffness Formulation}

\subsubsection{Flat Plate Element}

\subsubsection{Shape Functions}

Variations of the $u, v$ and $w$ displacements within the flat plate finite strip have been discussed in Section 3.3. For a one mode approximation in the longitudinal direction, the shape functions could be written as:

$$
\begin{array}{rlrl}
u & =N_{i}^{u} u_{i} ; & i=1,2 \\
v & =N_{i}^{v} v_{i} ; & i=1,2 \\
\text { and } & & \\
u & =N_{i}^{u r} v_{i} ; & i=1,3 \\
=N_{i}^{w} b \theta_{i} ; & i=2,4
\end{array}
$$

where 


$$
\begin{aligned}
& N_{1}^{u}=(1-\eta) g_{1}^{u}(\xi) \\
& N_{2}^{u}=\eta g_{1}^{u}(\xi) \\
& N_{1}^{v}=(1-\eta) g_{1}^{v}(\xi) \\
& N_{2}^{v}=\eta g_{1}^{v}(\xi) \\
& N_{1}^{w}=\left(1-3 \eta^{2}+2 \eta^{3}\right) g_{1}^{w}(\xi) \\
& N_{2}^{w}=\left(\eta-2 \eta^{2}+\eta^{3}\right) b g_{1}^{w}(\xi) \\
& N_{3}^{w}=\left(3 \eta^{2}-2 \eta^{3}\right) g_{1}^{w}(\xi) \\
& N_{4}^{w}=\left(\eta^{3}-\eta^{2}\right) b g_{1}^{w}(\xi)
\end{aligned}
$$

and $u_{11}, u_{21}, v_{11}, v_{21}, w_{11}, \theta_{11}, w_{21}, \theta_{21}$ in equation ( 3.6 ) are replaced by $u_{1}, u_{2}$, $v_{1}, v_{2}, w_{1}, \theta_{1}, w_{2}, \theta_{2}$.

In matrix notation Eq.( 3.19 ) can be represented by

$$
\left\{\begin{array}{c}
u \\
v \\
w
\end{array}\right\}=[N]\left\{\delta_{e}\right\}
$$

where $\left\{\delta_{e}\right\}$ is the nodal displacement vector given by 


$$
\left\{\delta_{e}\right\}=\left\{\begin{array}{c}
u_{1} \\
v_{1} \\
w_{1} \\
\theta_{1} \\
u_{2} \\
v_{2} \\
w_{2} \\
\theta_{2}
\end{array}\right\}
$$

and $[N]$ is a matrix of shape functions given by

$$
[N]=\left[\begin{array}{cccccccc}
N_{1}^{u} & 0 & 0 & 0 & N_{2}^{u} & 0 & 0 & 0 \\
0 & N_{1}^{v} & 0 & 0 & 0 & N_{2}^{v} & 0 & 0 \\
0 & 0 & N_{1}^{w} & N_{2}^{w} & 0 & 0 & N_{3}^{w} & N_{4}^{w}
\end{array}\right]
$$

Substituting Eq.( 3.20 ) into the strain-displacement relations of the plate given in Eq.( 3.8$)$, we obtain an explicit relation between strains and the nodal displacements. Defining

$$
\{\epsilon\}=\left\{\begin{array}{c}
\epsilon_{x} \\
\epsilon_{y} \\
\gamma_{x y}
\end{array}\right\}
$$


then,

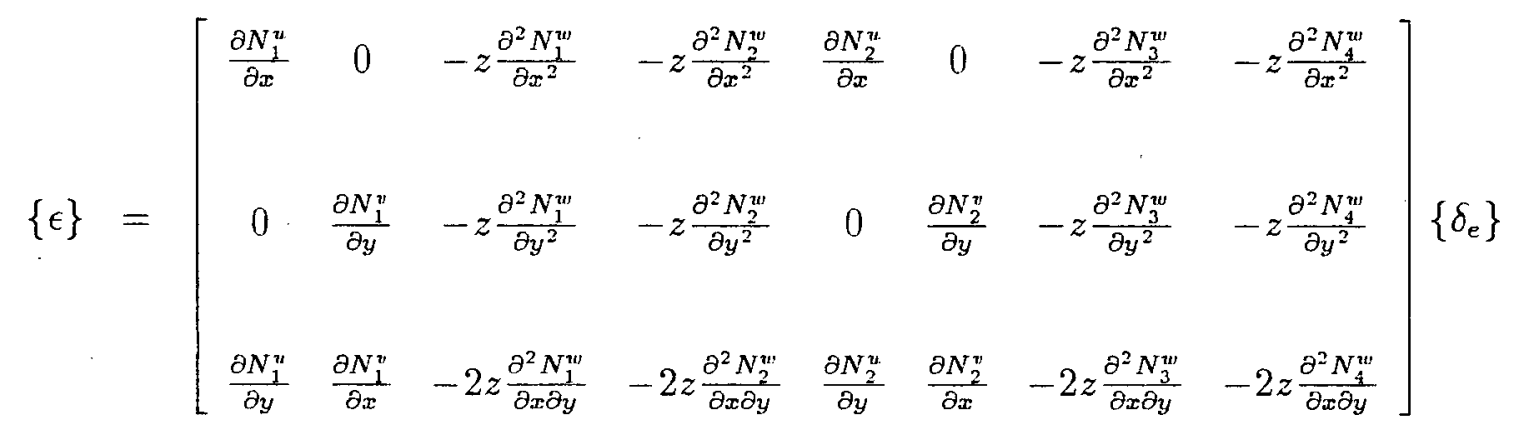

$$
\begin{aligned}
& +\left[\begin{array}{c}
\frac{1}{2} \frac{\partial N_{i}^{w}}{\partial x} \frac{\partial N_{j}^{w}}{\partial x} w_{i} w_{j} \\
\frac{1}{2} \frac{\partial N_{i}^{w}}{\partial y} \frac{\partial N_{j}^{w}}{\partial y} w_{i} w_{j} \\
\frac{1}{2}\left(\frac{\partial N_{i}^{w}}{\partial x} \frac{\partial N_{j}^{w}}{\partial y}+\frac{\partial N_{i}^{w}}{\partial x} \frac{\partial N_{j}^{w}}{\partial y}\right) w_{i} w_{j}
\end{array}\right] \\
& +\left[\begin{array}{c}
\frac{1}{2} \frac{\partial N_{k}^{v}}{\partial x} \frac{\partial N_{l}^{n}}{\partial x} v_{k} v_{l} \\
\frac{1}{2} \frac{\partial N_{k}^{n}}{\partial y} \frac{\partial N_{l}^{n}}{\partial y} v_{k} v_{l} \\
\frac{1}{2}\left(\frac{\partial N_{k}^{n}}{\partial x} \frac{\partial N_{l}^{\prime \prime}}{\partial y}+\frac{\partial N_{k}^{n}}{\partial x} \frac{\partial N_{l}^{n}}{\partial y}\right) v_{k} v_{l}
\end{array}\right]
\end{aligned}
$$

where $i, j=1,2,3,4$ and $k, l=1,2$.

\subsubsection{Virtual Work Principle}

From the strain displacement relations in Eq. $(3.24)$ it can be seen that the virtual strains are related to the virtual displacements by

$$
\{\tilde{\epsilon}\}=[[B]+[C]]\left\{\tilde{\delta}_{e}\right\}
$$

where, $\{\bar{\epsilon}\}$ is the vector of virtual strain and $\left\{\tilde{\delta}_{e}\right\}$ is the virtual displacement vector; 


$$
[B]=\left[\begin{array}{cccccccc}
\frac{\partial N_{1}^{u}}{\partial x} & 0 & -z \frac{\partial^{2} N_{1}^{w}}{\partial x^{2}} & -z \frac{\partial^{2} N_{2}^{w}}{\partial x^{2}} & \frac{\partial N_{2}^{u}}{\partial x} & 0 & -z \frac{\partial^{2} N_{3}^{w}}{\partial x^{2}} & -z \frac{\partial^{2} N_{4}^{w}}{\partial x^{2}} \\
0 & \frac{\partial N_{1}^{v}}{\partial y} & -z \frac{\partial^{2} N_{1}^{w \prime}}{\partial y^{2}} & -z \frac{\partial^{2} N_{2}^{w \prime}}{\partial y^{2}} & 0 & \frac{\partial N_{2}^{v}}{\partial y} & -z \frac{\partial^{2} N_{3}^{w}}{\partial y^{2}} & -z \frac{\partial^{2} N_{4}^{w \prime}}{\partial y^{2}} \\
\frac{\partial N_{1}^{u}}{\partial y} & \frac{\partial N_{1}^{\prime \prime}}{\partial x} & -2 z \frac{\partial^{2} N_{1}^{w \prime}}{\partial x \partial y} & -2 z \frac{\partial^{2} N_{2}^{w}}{\partial x \partial y} & \frac{\partial N_{2}^{u}}{\partial y} & \frac{\partial N_{2}^{\eta}}{\partial x} & -2 z \frac{\partial^{2} N_{3}^{w \prime}}{\partial x \partial y} & -2 z \frac{\partial^{2} N_{4}^{w}}{\partial x \partial y}
\end{array}\right]
$$

and,

$$
\begin{aligned}
& {\left[\begin{array}{ccc}
0 & \frac{1}{2} \frac{\partial N_{1}^{v}}{\partial x} \frac{\partial N_{l}^{v}}{\partial x} v_{l} & \frac{1}{2} \frac{\partial N_{1}^{w}}{\partial x} \frac{\partial N_{j}^{w \prime}}{\partial x} w_{j} \\
0 & \frac{1}{2} \frac{\partial N_{1}^{v}}{\partial y} \frac{\partial N_{l}^{u}}{\partial y} v_{l} & \frac{1}{2} \frac{\partial N_{1}^{w}}{\partial y} \frac{\partial N_{j}^{w}}{\partial y} w_{j} \\
0 & \frac{1}{2}\left(\frac{\partial N_{1}^{v}}{\partial x} \frac{\partial N_{l}^{v}}{\partial y}+\frac{\partial N_{1}^{u}}{\partial x} \frac{\partial N_{l}^{v}}{\partial y}\right) v_{l} & \frac{1}{2}\left(\frac{\partial N_{1}^{w}}{\partial x} \frac{\partial N_{j}^{w}}{\partial y}+\frac{\partial N_{1}^{w}}{\partial y} \frac{\partial N_{j}^{w}}{\partial x}\right) u_{j}
\end{array}\right.} \\
& \frac{1}{2} \frac{\partial N_{2}^{w}}{\partial x} \frac{\partial N_{j}^{w}}{\partial x} w_{j} \quad . \quad 0 \quad \frac{1}{2} \frac{\partial N_{2}^{w}}{\partial x} \frac{\partial N_{l}^{v}}{\partial x} v_{l}
\end{aligned}
$$

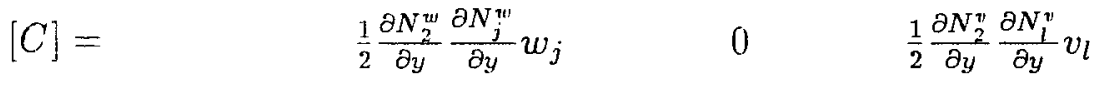

$$
\begin{aligned}
& \frac{1}{2}\left(\frac{\partial N_{2}^{u w}}{\partial x} \frac{\partial N_{j}^{w}}{\partial y}+\frac{\partial N_{2}^{w}}{\partial y} \frac{\partial N_{j}^{w}}{\partial x}\right) w_{j} \quad 0 \quad \frac{1}{2}\left(\frac{\partial N_{2}^{v}}{\partial x} \frac{\partial N_{l}^{v}}{\partial y}+\frac{\partial N_{2}^{v}}{\partial x} \frac{\partial N_{l}^{v}}{\partial y}\right) v_{l} \\
& \frac{1}{2} \frac{\partial N_{3}^{w:}}{\partial x} \frac{\partial N_{j}^{w}}{\partial x} w_{j} \quad \frac{1}{2} \frac{\partial N_{4}^{w}}{\partial x} \frac{\partial N_{j}^{w}}{\partial x} w_{j} \\
& \frac{1}{2} \frac{\partial N_{3}^{u \prime}}{\partial y} \frac{\partial N_{j}^{w \prime}}{\partial y} w_{j} \quad \quad \frac{1}{2} \frac{\partial N_{i}^{w \prime}}{\partial y} \frac{\partial N_{j}^{w \prime}}{\partial y} w_{j} \\
& \frac{1}{2}\left(\frac{\partial N_{3}^{w \prime \prime}}{\partial x} \frac{\partial N_{j}^{w \prime}}{\partial y}+\frac{\partial N_{3}^{w}}{\partial y} \frac{\partial N_{j}^{\prime \prime \prime}}{\partial x}\right) w_{j} \quad \frac{1}{2}\left(\frac{\partial N_{4}^{w w}}{\partial x} \frac{\partial N_{j}^{w}}{\partial y}+\frac{\partial N_{4}^{w \prime}}{\partial y} \frac{\partial N_{j}^{w \prime}}{\partial x}\right) w_{j}
\end{aligned}
$$


It can easily be observed that the $[B]$ matrix is independent of the nodal displacements while the $[C]$ matrix is a linear function of $\{\delta\}$. Using the principle of virtual work, the equation of equilibrium of a single flat plate finite strip can be written as

$$
\int_{V}\{\tilde{\epsilon}\}^{T}\{\sigma\} d v o l=\left\{\tilde{\delta}_{e}\right\}^{T}\{\mathbf{p}\}
$$

where

$$
\{\sigma\}=\left\{\begin{array}{c}
\sigma_{x} \\
\sigma_{y} \\
\tau_{x y}
\end{array}\right\}
$$

and $\{\mathbf{p}\}$ is a consistent load vector calculated from the shape functions $[N]$.

Substituting Eq.( 3.25 ) into the virtual work equation given by by Eq.( 3.27 ) results in the equilibrium equation for a single finite strip given by

$$
\int_{V}[[B]+[C]]^{T}\{\sigma\} d v o l=\{\mathbf{p}\}
$$

The equilibrium equation given by Eq.( 3.29$)$ for a single finite strip represents the stiffness matrix and the corresponding load vector in a local co-ordinate system. Proceeding in the same manner a local stiffness and a 'consistent' load for each strip can be formulated. However, for the final analysis, the stiffness matrix and the load vector need to be transformed from the local to the global system.

Transforming the element displacement and the element load vector from the global to the local co-ordinates

$$
\left\{\delta_{L}\right\}^{e}=\left[T_{e}\right]\left\{\delta_{G}\right\}^{e}
$$

and 


$$
\left\{P_{L}\right\}^{e}=\left[T_{e}\right]\left\{P_{G}\right\}^{e}
$$

where,

$$
\left[T_{e}\right]=\left[\begin{array}{cccccccc}
1 & 0 & 0 & 0 & 0 & 0 & 0 & 0 \\
0 & \cos \theta & \sin \theta & 0 & 0 & 0 & 0 & 0 \\
0 & -\sin \theta & \cos \theta & 0 & 0 & 0 & 0 & 0 \\
0 & 0 & 0 & 1 & 0 & 0 & 0 & 0 \\
0 & 0 & 0 & 0 & 1 & 0 & 0 & 0 \\
0 & 0 & 0 & 0 & 0 & \cos \theta & \sin \theta & 0 \\
0 & 0 & 0 & 0 & 0 & -\sin \theta & \cos \theta & 0 \\
0 & 0 & 0 & 0 & 0 & 0 & 0 & 1
\end{array}\right] .
$$

and $\theta$ is the angle between the local and the global co-ordinate axis of the element.

The stiffness matrix is formulated in the local co-ordinate system. Transforming from the local to the global co-ordinates

$$
\begin{aligned}
\left\{P_{G}\right\}^{e} & =\left[T_{e}\right]^{-1}\left\{P_{L}\right\}^{e} \\
& =\left[T_{e}\right]^{-1}\left\{k_{L}\right\}^{e}\left[T_{e}\right]\left\{\delta_{G}\right\}^{e}
\end{aligned}
$$

Therefore,

$$
\left\{k_{G}\right\}^{e}=\left[T_{e}\right]^{-1}\left\{k_{L}\right\}^{e}\left[T_{e}\right]
$$

Since the work done in the two co-ordinate systems must be the same, the transformation matrix $\left[T_{e}\right]$ must be orthogonal, i.e.

$$
\left[T_{e}\right]^{-1}=\left[T_{e}\right]^{T}
$$


Hence,

$$
\left\{k_{G}\right\}^{e}=\left[T_{e}\right]^{T}\left\{k_{L}\right\}^{e}\left[T_{e}\right]
$$

Similarly, the load vector after transformation will be given by

$$
\begin{aligned}
\left\{P_{G}\right\}^{e} & =\left[T_{e}\right]^{-1}\left\{P_{e}\right\}^{e} \\
& =\left[T_{e}\right]^{T}\left\{P_{L}\right\}^{e}
\end{aligned}
$$

Assembling the stiffness matrix for each element in this manner, a structural stiffness matrix can be obtained. Then, the equilibrium equation for the whole structure can be represented by

$$
\begin{aligned}
\sum_{\text {allstrips }} \int_{V}\left[T_{e}\right]^{T}[[B]+[C]]^{T}\left[T_{e}\right]\{\sigma\} \text { dvol } & =\sum_{\text {allstrips }}\left[T_{e}\right]^{T}\left\{p_{e}\right\} \\
& =\{\mathbf{P}\}
\end{aligned}
$$

\subsubsection{Load Vector}

In the assumed finite strip discretization of a cylindrical shell using flat plate elements, an attempt has been made to model a continuously curved surface by small, flat elements. It is very important to model the loading on the shell surface appropriately. To represent a uniformly distributed load e.g. the self-weight of the shell, Zienkiewicz [29], argues that it is more consistent, with the physical aspect of replacing a curved surface by a collection of planes, to concentrate the distributed load as statically equivalent nodal forces. In the present formulation, the self-weight of the shell is modelled as being concentrated along the nodal lines, therefore, in the transverse direction, the load is lumped. The load vector is integrated in closed form with the appropriate shape functions in the longitudinal direction. Therefore, a pseudo-consistent load vector is generated for each element which is subsequently transformed by the transformation matrix given in Eq. ( 3.37$)$ to obtain the global load vector. 


\subsubsection{Curved Shell Strip I}

\subsubsection{Shape Functions}

The shape functions used in this formulation are identical to those used in the flat plate formulation. Substituting the shape functions from Eq.( 3.19$)$ into the straindisplacement relations of the cylindrical shell given by Eq.( 3.9$)$ gives

$$
\begin{aligned}
& {\left[\begin{array}{cccc}
\frac{\partial N_{1}^{u}}{\partial x} & 0 & -z \frac{\partial^{2} N_{2}^{u w}}{\partial x^{2}} & -z \frac{\partial^{2} N_{2}^{w}}{\partial x^{2}} \\
0 & \left(1+\frac{z}{R}\right) \frac{\partial N_{1}^{u}}{\partial y} & \frac{N_{1}^{w}}{R}-z \frac{\partial^{2} N_{1}^{u \prime}}{\partial y^{2}} & \frac{N_{2}^{u}}{R}-z \frac{\partial^{2} N_{2}^{u w}}{\partial y^{2}} \\
\frac{\partial N_{1}^{u}}{\partial y} & \left(1+\frac{z}{R}\right) \frac{\partial N_{1}^{u}}{\partial x} & -2 z \frac{\partial^{2} N_{1}^{w}}{\partial x \partial y} & -2 z \frac{\partial^{2} N_{2}^{w}}{\partial x \partial y}
\end{array}\right.} \\
& \{\epsilon\}= \\
& \frac{\partial N_{2}^{u}}{\partial x} \quad 0 \quad-z \frac{\partial^{2} N_{3}^{w}}{\partial x^{2}} \quad-z \frac{\partial^{2} N_{u}^{u}}{\partial x^{2}} \\
& 0 \quad\left(1+\frac{z}{R}\right) \frac{\partial N_{2}^{v}}{\partial y} \quad \frac{N_{3}^{u v}}{R}-z \frac{\partial^{2} N_{3}^{w u}}{\partial y^{2}} \quad \frac{N_{u}^{u v}}{R}-z \frac{\partial^{2} N_{u}^{u}}{\partial y^{2}} \quad\left\{\delta_{e}\right\}
\end{aligned}
$$

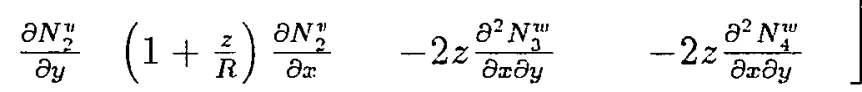

$$
\begin{aligned}
& +\left[\begin{array}{c}
\frac{1}{2} \frac{\partial N_{i}^{w}}{\partial x} \frac{\partial N_{j}^{w}}{\partial x} w_{i} w_{j} \\
\frac{1}{2} \frac{\partial N_{i}^{w}}{\partial y} \frac{\partial N_{j}^{w}}{\partial y} w_{i} w_{j} \\
\frac{1}{2}\left(\frac{\partial N_{i}^{w}}{\partial x} \frac{\partial N_{j}^{w_{j}^{w}}}{\partial y}+\frac{\partial N_{i}^{w}}{\partial x} \frac{\partial N_{j}^{w}}{\partial y}\right) w_{i} w_{j}
\end{array}\right] i, j=1,2,3,4
\end{aligned}
$$




\subsubsection{Virtual Work Principle}

As in the flat plate formulation, the virtual strains are related to the virtual displacements as in Eq.( 3.25$)$ where,

$$
\begin{aligned}
& {\left[\begin{array}{cccc}
\frac{\partial N_{1}^{u}}{\partial x} & 0 & -z \frac{\partial^{2} N_{1}^{u \prime}}{\partial x^{2}} & -z \frac{\partial^{2} N_{2}^{u w}}{\partial x^{2}} \\
0 & \left(1+\frac{z}{R}\right) \frac{\partial N_{1}^{v}}{\partial y} & \frac{N_{1}^{u}}{R}-z \frac{\partial^{2} N_{1}^{w}}{\partial y^{2}} & \frac{N_{2}^{w}}{R}-z \frac{\partial^{2} N_{2}^{u \prime}}{\partial y^{2}} \\
\frac{\partial N_{1}^{u}}{\partial y} & \left(1+\frac{z}{R}\right) \frac{\partial N_{1}^{u}}{\partial x} & -2 z \frac{\partial^{2} N_{1}^{w}}{\partial x \partial y} & -2 z \frac{\partial^{2} N_{2}^{N_{2}^{u}}}{\partial x \partial y}
\end{array}\right.} \\
& {[B]=} \\
& \frac{\partial N_{2}^{u}}{\partial x} \quad 0 \quad-z \frac{\partial^{2} N_{3}^{w}}{\partial x^{2}} \quad-z \frac{\partial^{2} N_{u}^{u \prime}}{\partial x^{2}} \\
& 0 \quad\left(1+\frac{z}{R}\right) \frac{\partial N_{2}^{v}}{\partial y} \quad \frac{N_{3}^{w}}{R}-z \frac{\partial^{2} N_{3}^{w}}{\partial y^{2}} \quad \frac{N_{4}^{w}}{R}-z \frac{\partial^{2} N_{u}^{u v}}{\partial y^{2}} \\
& \frac{\partial N_{2}^{u}}{\partial y}\left(1+\frac{z}{R}\right) \frac{\partial N_{z}^{u}}{\partial x} \quad-2 z \frac{\partial^{2} N_{3}^{u \prime}}{\partial x \partial y} \quad-2 z \frac{\partial^{2} N_{y}^{u}}{\partial x \partial y}
\end{aligned}
$$

and 


$$
\begin{aligned}
& {\left[\begin{array}{cccc}
0 & 0 & \frac{1}{2} \frac{\partial N_{1}^{w \prime}}{\partial x} \frac{\partial N_{j}^{u \prime}}{\partial x} w_{j} & \frac{1}{2} \frac{\partial N_{2}^{u \prime}}{\partial x} \frac{\partial N_{j}^{w \prime}}{\partial x} w_{j} \\
0 & 0 & \frac{1}{2} \frac{\partial N_{1}^{u \prime}}{\partial y} \frac{\partial N_{j}^{u \prime}}{\partial y} w_{j} & \frac{1}{2} \frac{\partial N_{2}^{w \prime}}{\partial y} \frac{\partial N_{j}^{w \prime}}{\partial y} w_{j} \\
0 & 0 & \frac{1}{2}\left(\frac{\partial N_{1}^{w u}}{\partial x} \frac{\partial N_{j}^{w \prime}}{\partial y}+\frac{\partial N_{1}^{u \prime}}{\partial y} \frac{\partial N_{j}^{w \prime}}{\partial x}\right) w_{j} & \frac{1}{2}\left(\frac{\partial N_{2}^{w \prime}}{\partial x} \frac{\partial N_{j}^{w}}{\partial y}+\frac{\partial N_{2}^{w \prime \prime}}{\partial y} \frac{\partial N_{j}^{w \prime}}{\partial x}\right) w_{j}
\end{array}\right.} \\
& {[C]=} \\
& \left.\begin{array}{cccc}
0 & 0 & \frac{1}{2} \frac{\partial N_{3}^{w}}{\partial x} \frac{\partial N_{j}^{w \prime}}{\partial x} w_{j} & \frac{1}{2} \frac{\partial N_{4}^{w \prime}}{\partial x} \frac{\partial N_{j}^{w}}{\partial x} w_{j} \\
0 & 0 & \frac{1}{2} \frac{\partial N_{3}^{w \prime}}{\partial y} \frac{\partial N_{j}^{w \prime}}{\partial y} w_{j} & \frac{1}{2} \frac{\partial N_{4}^{w \prime}}{\partial y} \frac{\partial N_{j}^{w \prime}}{\partial y} w_{j} \\
0 & 0 & \frac{1}{2}\left(\frac{\partial N_{3}^{w}}{\partial x} \frac{\partial N_{j}^{w}}{\partial y}+\frac{\partial N_{3}^{w \prime \prime}}{\partial y} \frac{\partial N_{j}^{w \prime}}{\partial x}\right) w_{j} & \frac{1}{2}\left(\frac{\partial N_{4}^{w \prime}}{\partial x} \frac{\partial N_{j}^{w \prime}}{\partial y}+\frac{\partial N_{4}^{w \prime}}{\partial y} \frac{\partial N_{j}^{w \prime}}{\partial x}\right) w_{j}
\end{array}\right]
\end{aligned}
$$

Proceeding in the same manner as in the flat plate formulation, the equilibrium equation for a single finite strip is given by Eq.( 3.29$)$. However, in this formulation, the local and the global co-ordinate systems coincide. Therefore, there is no need for a transformation of co-ordinates. By satisfying inter-element compatibility along the nodal lines, the equilibrium of the cylindrical shell can be represented by

$$
\begin{aligned}
\sum_{\text {allstrips }} \int_{V}[[B]+[C]]^{T}\{\sigma\} d v o l & =\sum_{\text {allstrips }}\left\{\mathbf{p}_{\mathbf{e}}\right\} \\
& =\{\mathbf{P}\}
\end{aligned}
$$

where $\mathbf{P}$ is the structural load vector.

\subsubsection{Load Vector}

In this formulation, the cylindrical shell has been represented exactly relative to its geometry and from considerations of strain-displacement relations. The load vector is 
consequently, 'consistent'.

\subsubsection{Curved Shell II}

\subsubsection{Shape Functions}

The variations of the $u, v$ and $w$ displacements within the curved shell finite strip formulation are given by Eq.( 3.7$)$.

For a one mode approximation in the longitudinal direction, the shape functions can be written as:

$$
\begin{array}{rlrl}
u & =N_{i}^{u} u_{i} ; & & i=1,2,3 \\
v & =N_{i}^{v} v_{i} ; & i=1,2,3 \\
\text { and } & & \\
w=N_{i}^{w} w_{i} ; & i=1,3 \\
& =N_{i}^{w} b \theta_{i} ; & & i=2,4
\end{array}
$$

where 


$$
\begin{aligned}
& N_{1}^{u}=\left(1-3 \eta+2 \eta^{2}\right) g_{1}^{u}(\xi) \\
& N_{2}^{u}=\left(4 \eta-4 \eta^{2}\right) g_{1}^{u}(\xi) \\
& N_{3}^{u}=\left(-\eta+2 \eta^{2}\right) g_{1}^{u}(\xi) \\
& N_{1}^{v}=\left(1-3 \eta+2 \eta^{2}\right) g_{1}^{v}(\xi) \\
& N_{2}^{v}=\left(4 \eta-4 \eta^{2}\right) g_{1}^{v}(\xi) \\
& N_{3}^{v}=\left(-\eta+2 \eta^{2}\right) g_{1}^{v}(\xi) \\
& N_{1}^{w}=\left(1-3 \eta^{2}+2 \eta^{3}\right) g_{1}^{w}(\xi) \\
& \dot{N_{2}^{w}}=\left(\eta-2 \eta^{2}+\eta^{3}\right) b g_{1}^{w}(\xi) \\
& N_{3}^{w}=\left(3 \eta^{2}-2 \eta^{3}\right) g_{1}^{w}(\xi) \\
& N_{4}^{w}=\left(\eta^{3}-\eta^{2}\right) b g_{1}^{w}(\xi)
\end{aligned}
$$

and $u_{11}, u_{21}, u_{31}, v_{11}, v_{21}, v_{31}, w_{11}, \theta_{11}, w_{21}, \theta_{21}$ in equation ( 3.7 ) are replaced by $u_{1}, u_{2}, u_{3}, v_{1}, v_{2}, v_{3}, w_{1}, \theta_{1}, w_{2}, \theta_{2}$.

In matrix notation Eq.( 3.42 ) can be represented by 


$$
\left\{\begin{array}{c}
u \\
v \\
w
\end{array}\right\}=[N]\left\{\delta_{e}\right\}
$$

where $\left\{\delta_{e}\right\}$ is the nodal displacement vector given by

$$
\left\{\delta_{e}\right\}=\left\{\begin{array}{c}
u_{1} \\
v_{1} \\
w_{1} \\
\theta_{1} \\
u_{2} \\
v_{2} \\
u_{3} \\
v_{3} \\
w_{2} \\
\theta_{2}
\end{array}\right\}
$$

and $[N]$ is a matrix of shape functions given by

$$
[N]=\left[\begin{array}{cccccccccc}
N_{1}^{u} & 0 & 0 & 0 & N_{2}^{u} & 0 & N_{3}^{u} & 0 & 0 & 0 \\
0 & N_{1}^{v} & 0 & 0 & 0 & N_{2}^{v} & 0 & N_{3}^{v} & 0 & 0 \\
0 & 0 & N_{1}^{w} & N_{2}^{w} & 0 & 0 & 0 & 0 & N_{3}^{w} & N_{4}^{w}
\end{array}\right]
$$


Substituting Eq.( 3.43 ) into the strain-displacement relations of the plate given in Eq.( 3.9 ), we obtain an explicit relation between strains and the nodal displacements given by

$$
\begin{aligned}
& {\left[\begin{array}{lllll}
\frac{\partial N_{1}^{u}}{\partial x} & 0 & -z \frac{\partial^{2} N_{1}^{u !}}{\partial \boldsymbol{x}^{2}} & -z \frac{\partial^{2} N_{2}^{u \prime}}{\partial x^{2}} & \frac{\partial N_{2}^{u}}{\partial x}
\end{array}\right.} \\
& 0 \quad\left(1+\frac{z}{R}\right) \frac{\partial N_{1}^{\nu}}{\partial y} \quad \frac{N_{1}^{w}}{R}-z \frac{\partial^{2} N_{1}^{w}}{\partial y^{2}} \frac{N_{2}^{w}}{R}-z \frac{\partial^{2} N_{2}^{w}}{\partial y^{2}} \quad 0
\end{aligned}
$$

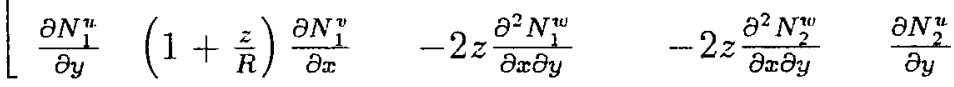

$$
\begin{aligned}
& \{\epsilon\}=
\end{aligned}
$$

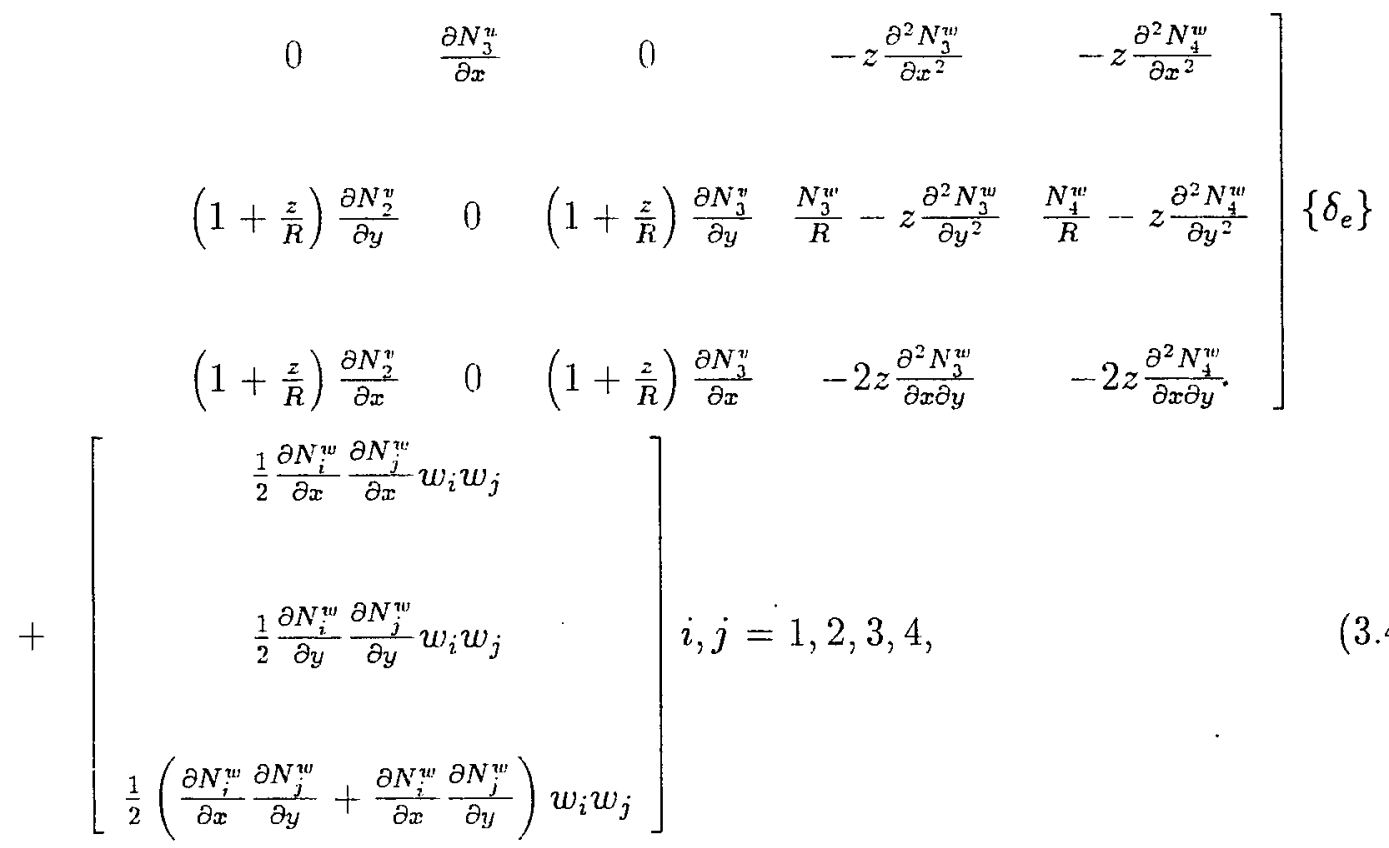

\subsubsection{Virtual Work Principle}

As in the Curved Shell I formulation, the virtual strains are related to the virtual displacements as in Eq.( 3.25$)$ where, 


$$
\begin{aligned}
& {\left[\begin{array}{ccccc}
\frac{\partial N_{1}^{u}}{\partial x} & 0 & -z \frac{\partial^{2} N_{1}^{w}}{\partial x^{2}} & -z \frac{\partial^{2} N_{2}^{w}}{\partial x^{2}} & \frac{\partial N_{2}^{u}}{\partial x} \\
0 & \left(1+\frac{z}{R}\right) \frac{\partial N_{1}^{\prime \prime}}{\partial y} & \frac{N_{1}^{u \prime}}{R}-z \frac{\partial^{2} N_{1}^{w}}{\partial y^{2}} & \frac{N_{2}^{w}}{R}-z \frac{\partial^{2} N_{2}^{w \prime}}{\partial y^{2}} & 0 \\
\frac{\partial N_{1}^{u}}{\partial y} & \left(1+\frac{z}{R}\right) \frac{\partial N_{1}^{v}}{\partial x} & -2 z \frac{\partial^{2} N_{1}^{w}}{\partial x \partial y} & -2 z \frac{\partial^{2} N_{2}^{w}}{\partial x \partial y} & \frac{\partial N_{2}^{u}}{\partial y}
\end{array}\right.} \\
& {[B]=} \\
& \left.\begin{array}{ccccc}
0 & \frac{\partial N_{3}^{u}}{\partial x} & 0 & -z \frac{\partial^{2} N_{3}^{w}}{\partial x^{2}} & -z \frac{\partial^{2} N_{4}^{w}}{\partial x^{2}} \\
\left(1+\frac{z}{R}\right) \frac{\partial N_{2}^{v}}{\partial y} & 0 & \left(1+\frac{z}{R}\right) \frac{\partial N_{3}^{v}}{\partial y} & \frac{N_{3}^{w \prime}}{R}-z \frac{\partial^{2} N_{3}^{w}}{\partial y^{2}} & \frac{N_{4}^{w}}{R}-z \frac{\partial^{2} N_{3}^{u \prime}}{\partial y^{2}} \\
\left(1+\frac{z}{R}\right) \frac{\partial N_{2}^{\prime \prime}}{\partial x} & 0 & \left(1+\frac{z}{R}\right) \frac{\partial N_{3}^{v}}{\partial x} & -2 z \frac{\partial^{2} N_{3}^{u \prime}}{\partial x \partial y} & -2 z \frac{\partial^{2} N_{4}^{w \prime}}{\partial x \partial y}
\end{array}\right]
\end{aligned}
$$

and,

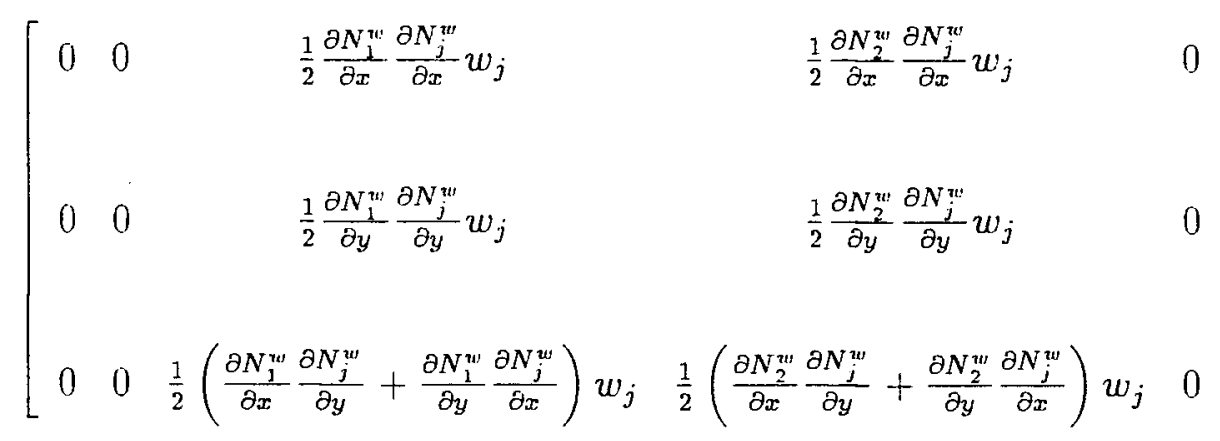

$[C]=$
000
$\frac{1}{2} \frac{\partial N_{3}^{w}}{\partial x} \frac{\partial N_{j}^{w}}{\partial x} u_{j}$
$\frac{1}{2} \frac{\partial N_{q}^{w}}{\partial x} \frac{\partial N_{j}^{w}}{\partial x} w_{j}$
$\begin{array}{lll}0 & 0 & 0\end{array}$
$\frac{1}{2} \frac{\partial N_{3}^{\prime \prime}}{\partial y} \frac{\partial N_{j}^{w}}{\partial y} w_{j}$
$\frac{1}{2} \frac{\partial N_{4}^{w}}{\partial y} \frac{\partial N_{j}^{w}}{\partial y} w_{j}$

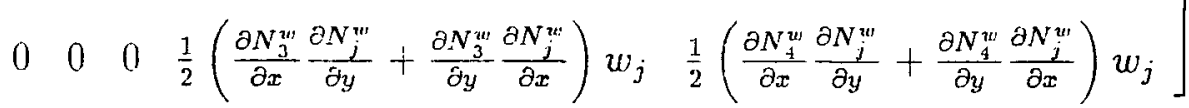


Assembling the equilibrium equation for each finite strip given by Eq.( 3.28 ) and satisfying inter-element compatibility, the equilibrium of the cylindrical shell will be given by Eq.( 3.40$)$. As in the formulation of Curved Shell I, there is no need for a transformation as the local and the global co-ordinate systems coincide.

\subsubsection{Load Vector}

In this formulation, the shape functions along the transverse direction have been upgraded from linear to quadratic for the in-plane displacements $u$ and $v$. Consequently, a consistent load vector is obtained by replacing the linear shape functions in the Curved Shell I formulation by quadratic ones and integrating in closed form.

The terms in the load vector for a self-weight analysis are given in Appendix A.

\subsection{Newton-Raphson Iterative Procedure}

The resulting equilibrium equations are non-linear in the nodal displacements and need to be solved using an iterative procedure.

Let us represent the equilibrium of a finite strip as

$$
\{f(\Delta)\}=p
$$

Expanding $f$ in Taylor series around a known solution $\Delta_{0}$, we obtain

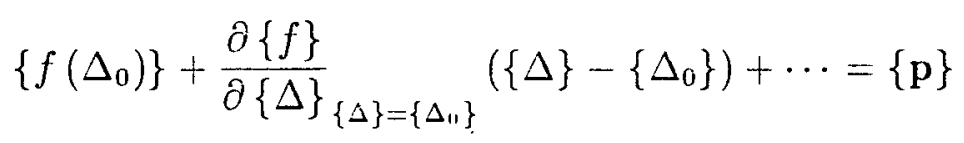


Rearranging terms, we get

$$
[K]\left\{\delta \Delta_{0}\right\}=p-\left\{f\left(\Delta_{0}\right)\right\}
$$

where, $[K]$ is the tangent stiffness matrix given by

$$
[K]=\frac{\partial\{f\}}{\partial\{\Delta\}_{\{\Delta\}=\left\{\Delta_{11}\right\}}}
$$

and $\left\{\delta \Delta_{0}\right\}$ is the incremental nodal displacement vector.

From Eq.( 3.50$)$ the tangent stiffness matrix is determined at each iteration by differentiating the equilibrium function $f$ with respect to the nodal displacement vector $\{\Delta\}_{i}$ at the end of the ith iteration.

Differentiating,

$$
\begin{aligned}
\frac{\partial\{f\}}{\partial\{\Delta\}} & =\frac{\partial}{\partial\{\Delta\}} \int_{V}[[B]+[C]]^{T}\{\sigma\} d v o l \\
& =\int_{V} \frac{\partial}{\partial\{\Delta\}}[[B]+[C]]^{T}\{\sigma\} d v o l
\end{aligned}
$$

Assuming that the differentiation with respect to the nodal displacement vector can be interchanged with the integration over the volume, we then obtain,

$$
\begin{aligned}
\frac{\partial}{\partial\{\Delta\}}[[B]+[C]]^{T}\{\sigma\} & =\frac{\partial[[B]+[C]]^{T}}{\partial\{\Delta\}}\{\sigma\}+[[B]+[C]]^{T} \frac{\partial\{\sigma\}}{\partial\{\Delta\}} \\
& =\frac{\partial[C]^{T}}{\partial\{\Delta\}}\{\sigma\}+[[B]+[C]]^{T} \frac{\partial\{\sigma\}}{\partial\{\epsilon\}} \frac{\partial\{\epsilon\}}{\partial\{\Delta\}}
\end{aligned}
$$

since $[B]$ is independent of $\{\Delta\}$. 
The first term on the right hand side of Eq.( 3.52$)$ can be expressed as:

$$
\left(\frac{\partial[C]^{T}}{\partial\{\Delta\}}\right)\{\sigma\}=[Q]
$$

where

$$
Q_{i j}=\sum_{1}^{3} \frac{\partial\left(C^{T}\right)_{i k}}{\partial\{\Delta\}_{j}} \sigma_{k}
$$

The derivation of $Q_{i j}$ is presented in Appendix B.

From Eq.( 3.52$)$

$$
\frac{\partial\{\sigma\}}{\partial\{\epsilon\}}=\left[D_{T}\right]
$$

and,

$$
\frac{\partial\{\epsilon\}}{\partial\{\Delta\}}=[[B]+[C]]
$$

Rewriting Eq.( 3.52 ) by substituting Eqs.( 3.55$),(3.56)$ and ( 3.53 ), we obtain

$$
[K]=\int_{V}\left\{[[B]+[C]]^{T}\left[D_{T}\right][[B]+[C]+[Q]]\right\} d v o l
$$

The Newton-Raphson iterative scheme is now carried out as follows:

1. Calculate $f\left(\Delta_{0}\right)$ from the previous iteration or load step. (For the first load increment, $f\left(\Delta_{0}\right)$ is calculated from the known stiffness matrix and the linear displacement vector in local co-ordinates). 
2. Compute the tangent stiffness matrix $[K]$ from Eq.( 3.57$)$.

3. Using Eq.( 3.49$)$ solve for $\left\{\delta \Delta_{i}\right\}$.

4. Then $\{\Delta\}_{i+1}$ at the $(i+1)$ th iteration will be given by

$$
\{\Delta\}_{i+1}=\{\Delta\}_{i}+\delta \Delta_{i}
$$

5. Repeat Steps 1-4 till the desired convergence is achieved.

\subsection{Numerical Integration}

The volume integral in Eq.( 3.57 ) cannot be integrated analytically in closed form as the stress-strain relationship is not known explicitly. Consequently, a numerical integration procedure needs to be adopted. In the present analysis, Gaussian Quadrature is used. The Gaussian Quadrature formulae can integrate a polynomial function $f(x)$ of degree $(2 n-1)$ exactly as a weighted mean of its $n$ particular values at specified locations. The number of points necessary in any one direction is dependent on the complexity of the function $f(x)$.

Transforming the co-ordinates in the three directions to their non-dimensional form, the integral given by Eq.( 3.57 ) will be evaluated using. Gaussian Quadrature as

$$
\begin{aligned}
I & =\int_{-1}^{1} \int_{-1}^{1} \int_{-1}^{1} f(\xi, \eta, \zeta) d \xi d \eta d \zeta \\
& \approx \sum_{i} \sum_{j} \sum_{k} W_{i} W_{j} W_{k} f\left(\xi_{i}, \eta_{j}, \zeta_{k}\right)
\end{aligned}
$$

where $W_{i}, W_{j}$ and $W_{k}$ are the weighting factors and $\xi_{i}, \eta_{j}$ and $\zeta_{k}$ denote the sampling points. The number of integrating points in the three directions are $i, j$ and $k$ respectively.

In the longitudinal direction, the displacement variations consist of circular and hyperbolic functions. In the integration of the function $f(x)$ a typical higher order term 
that will be encountered will look like $\sin ^{2} \pi \xi, \cos ^{2} \pi \xi, \sinh ^{2} \beta \xi$ or $\cosh \beta \xi \sinh \beta \xi$ in elastic small deflection analysis and $\sin ^{4} \pi \xi, \sinh \beta \xi \cosh \beta \xi$ etc in elastic large deflection analysis for a one mode solution. From an investigation carried out by Abayakoon [24], it was found that by using 5 integration points in the longitudinal direction, the error in numerical integration in the elastic large deflection analysis was only about $0.5 \%$.

The displacement variations in the Flat Plate formulation are linear for $u$ and $v$ and cubic for $w$ in the transverse direction. Therefore, in Eq. ( 3.57$)$, the terms will be quadratic polynomials in elastic small or large deflection analysis. Therefore, a 2-point Gaussian integration will be sufficient in the transverse direction. In the cylindrical shell theory, the terms in the corresponding integral will be of order 4 due to the presence of an extra term in the strain displacement relations i.e. $\frac{w}{R}$. Therefore, a 3 point Gaussian integration in the transverse direction is adopted. A 2-point Gaussian integration is used in the elastic analysis through the thickness of the cylindrical shell.

When the shell material becomes non-linear, yielding of the material extends through the thickness. From a study conducted by Wu and Witmer [30], for thin beams of rectangular cross-section, it was found that a 4-point Gaussian integration was sufficient to give an accurate representation of the non-linear stress distribution. In the present study, a 4-point Gaussian integration scheme through the thickness is adopted in the elastic-plastic analysis.

\subsection{Computer Implementation}

The finite strip formulation for the flat plate and the curved shell models is implemented on the mainframe AMDHAL 5850 computers. The program is written in FORTRAN and test runs are carried out to verify the accuracy of the procedure. 
The user has the option to specify the number of modes to be chosen for the displacement components $u, v$ and $w$. The number of Gaussian integration points are chosen by the program depending on the type of analysis - elastic or plastic and on the number of modes chosen. The final set of equations is solved using Gaussian elimination. The convergence of the solution algorithm is determined by one of two criteria as follows:

1. In the maximum norm criterion, the solution is converged if,

$$
\left|\frac{\delta \Delta_{i}}{\Delta_{i}}\right|_{\max } \leq T O L E R_{1}
$$

where $\Delta_{i}$ is the displacement solution for the nodal variable $i$, and $\delta \Delta_{i}$ is the correction for that variable in the present iteration. TOLER $R_{1}$ is the acceptable level of tolerance specified by the user for this criterion.

2. In the Euclidean norm criterion, the solution is converged if,

$$
\frac{\sum_{i=1}^{i=N}\left(\delta \Delta_{i}\right)^{2}}{\sum_{i=1}^{i=N}\left(\Delta_{i}\right)^{2}} \leq \text { TOLER } R_{2}^{2}
$$

where $N$ is the total number of nodal variables and $T O L E R_{2}$ is the acceptable level of tolerance for this criterion. 


\section{Chapter 4}

\section{Numerical Investigations}

\subsection{Introduction}

In Chapter 3, the three finite strip formulations were developed. To investigate the accuracy and viability of each formulation, several example problems have been tested. The mode shapes in the longitudinal direction of the strip are identified by indices $m,(n, k)$ and $p$ introduced in Eq. ( 3.2$),(3.3)$ and ( 3.4$)$. In this chapter, the following notation has been employed while illustrating the example problems.

$(1,1,1)+\left(3,,_{-}\right)$

$(2,1,1)+(4,-,-)$

$(2,1,1)+(-, 1,-)$ one mode analysis $\mathrm{m}=2, \mathrm{n}=1, \mathrm{p}=1$

one mode analysis $\mathrm{m}=1, \mathrm{n}=1, \mathrm{p}=1$

two modes for the $u$ displacement $\mathrm{m}=1$ and $\mathrm{m}=3$

one mode for the $v$ displacement, $\mathrm{n}=1$

one mode for the $w$ displacement, $\mathrm{p}=1$

two modes for the $u$ displacement $m=2$ and $m=4$

one mode for the $v$ displacement, $n=1$

one mode for the $w$ displacement, $\mathrm{p}=1$

one mode for the $u$ displacement $\mathrm{m}=2$

two modes for the $v$ displacement, $\mathrm{n}=1, \mathrm{k}=1$

one mode for the $u$ displacement, $\mathrm{p}=1$ 
The first stage of the investigation was to carry out test runs on beam problems of inclined cross-sections for which the analytical solutions could easily be worked out. Such an investigation would reveal the correctness of the transformation matrix used in the flat plate finite strip formulation. The results from the finite strip analysis are compared with the analytical solutions in Section 4.2.

The shallow shell roof problem has been frequently used to test particular shell finite elements. The configuration of the test shell-roof problem is illustrated in Fig. 4.2. In all the finite strip formulations the analysis was carried out for 2 different boundary conditions at the curved edges - simply supported and clamped. In Section 4.3, various aspects of the flat plate finite strip analysis are discussed with respect to the shell-roof problem. The problem is initially examined for the linear elastic case. Effect of large displacements is also investigated. The vertical displacements at the mid-span of the free edge and at the crown and the strain energy, are compared with the analytical and numerical solutions. In addition, the stress and moment distributions at critical sections are compared with the results of finite-element procedures. Some comments on the accuracy of the flat plate formulation have also been made in this Section.

Section 4.4 includes the numerical investigations of the shell-roof problem using Curved Shell strips I. In addition to the comparison of the vertical displacements at the crown and the mid-span of the free edge, with other numerical procedures, the investigation extends to the determination of the number of finite strips required to adequately model the cylindrical panel under consideration. The accuracy of displacements and convergence of strain energy with the increase in the number of finite strips is also investigated. This section concludes with a discussion of the 
results and some comments regarding the performance of the Curved Shell strip I. vis-a-vis the Flat Plate formulation.

In Section 4.5, the shallow shell-roof problem is analyzed using Curved Finite strips II. A detailed analysis is carried out to investigate the convergence of strain energy for the linear case. The conclusions based on the analysis enable us to determine the number of finite strips necessary to model the cylindrical panel with a. reasonable accuracy.

In the next stage of the investigation, a linear elastic analysis of a cylindrical shell loaded symmetrically with respect to its axis is carried out. Section 4.6 includes the results of the linear elastic analysis of a circular cylindrical shell subjected to uniform internal pressure are presented. The response of the shell is compared with the analytical solutions to obtain an estimate of the accuracy of the finite strip formulation. A detailed parametric study is also carried out to determine the range of applicability of the finite strip formulation to the analysis of cylindrical shell problems. Further investigations of the shell-roof problem were carried out by incorporating the effect of geometric and material non-linearities.

In Section 4.7, a detailed numerical investigation is carried out to analyze the shallow shell-roof problem using Curved Shell strips II for linear, elastic non-linear geometry, and non-linear material behavior. The vertical displacements at the midspan of the free edge and at the crown are compared with the results from other numerical procedures.

The succeeding sections include the analysis of the shell-roof problem with clamped boundary conditions at the curved edges. Section 4.8 presents the results of the shallow shell-roof problem with clamped curved boundaries. The results for the linear elastic case are compared with the results other finite-element procedures. 
Section 4.9 consists of the results of a detailed analysis of the clamped-shell problem discussed in Section 4.8 for the linear elastic, non-linear geometry and non-linear material. Some features of the investigation are also discussed with respect to the results of the classical shallow shell-roof problem in the absence of any comparisons for the non-linear analysis. The effect of clamping all the boundaries of the cylindrical shell panel is investigated in Section 4.10. The panel is subjected to uniform radial pressure. The linear elastic response is compared with the available results from finite element procedures. The effect of incorporating geometric nonlinearities is also investigated.

As discussed in Chapter 3, the longitudinal displacement modes of the finite strip are represented by continuously differentiable functions. Most of the analyses in Sections 4.2 to 4.9 have been carried out by using one mode of these functions for each of the displacement components $u, v$ and $w$. The effect of adding another in-plane mode is also investigated where it is considered necessary.

\subsection{Analysis of a Rectangular Beam}

The behavior of a simply supported rectangular beam with an inclined cross-section at $45^{\circ}$, subjected to a uniformly distributed load, was studied using a one-mode finite strip analysis. Only one displacement mode was employed for each of the three displacement components in the longitudinal direction. Therefore, in our notation, the analysis is $(1,1,1)$.

A Rayleigh-Ritz analysis can be carried out to obtain the modal solutions for this problem by minimizing the total potential energy. The potential energy of the beam can be represented by 


$$
V=\frac{1}{2} \int_{0}^{L}\left[E I\left(\frac{\partial^{2} w}{\partial x^{2}}\right)-2 p w\right] d x
$$

where,

$x$ is the distance measured along the length of the beam,

$L$ is the length of the beam,

$p$ is the uniformly distributed load,

$E I$ is the flexural rigidity of the beam, and

$w$ is the lateral deflection.

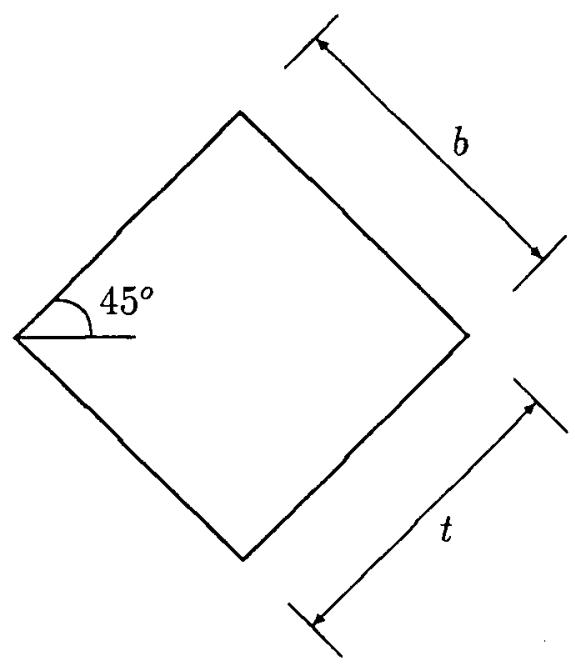

Figure 4.1: Cross-Section of the Simply Supported Beam

Let the vertical displacement be represented by

$$
w=w_{c} \sin \frac{\pi x}{L}
$$


Then, minimizing the potential energy of the beam with respect to the central deflection $w_{c}$, we obtain

$$
\begin{aligned}
w_{c} & =\frac{4 L^{4} p}{\pi^{5} E I} \\
& =\frac{5 L^{4} p}{382.5246 E I}
\end{aligned}
$$

This result is slightly higher than the central deflection obtained from the exact analysis i.e. $\frac{5 L^{4} p}{384 E I}$.

A comparison of the central deflection and strain energy from analytical methods is made with the results of the finite strip method in Table 4.1. The cross-sectional and material properties of the beam are also provided. A schematic sketch of the beam cross-section is also depicted in Fig. 4.1

Table 4.1: Linear Elastic Response of a Simply Supported Beam

$$
\begin{aligned}
L & =500 \mathrm{~mm} \\
b & =10 \mathrm{~mm} \\
t & =10 \mathrm{~mm} \\
E & =220000 \mathrm{~N} / \mathrm{mm}^{2} \\
\nu & =0.0 \\
p & =0.1 \mathrm{~N} / \mathrm{mm}^{2}
\end{aligned}
$$

\begin{tabular}{|c|c|c|c|}
\hline & Beam Theory & $\begin{array}{c}\text { One mode } \\
\text { analytical }\end{array}$ & $\begin{array}{c}\text { One mode } \\
\text { Finite Strip }\end{array}$ \\
\hline Central Deflection $(\mathrm{mm})$ & -3.1388 & -3.1509 & -3.1508 \\
Strain Energy $(\mathrm{Nmm})$ & 999.11 & 1002.93 & 1002.93 \\
\hline
\end{tabular}

The obvious conclusion which can be drawn from the one-mode linear elastic 
analysis of the beam problem is that the displacement components and the strain energy can be accurately predicted using a flat plate finite strip. Secondly, the numerical integration scheme is correct for this linear problem. Thirdly, the transformation matrix generated in Eq.( 3.32 ) for the transformation of co-ordinates from the local to global cartesian axes is correct.

\subsection{Analysis of the Shell-Roof problem using Flat Plate strips}

The shell-roof problem shown in Fig. 4.2 has been frequently used to test shell finite-elements. The curved edges of the cylindrical shell are diaphram supported while the straight edges are free.

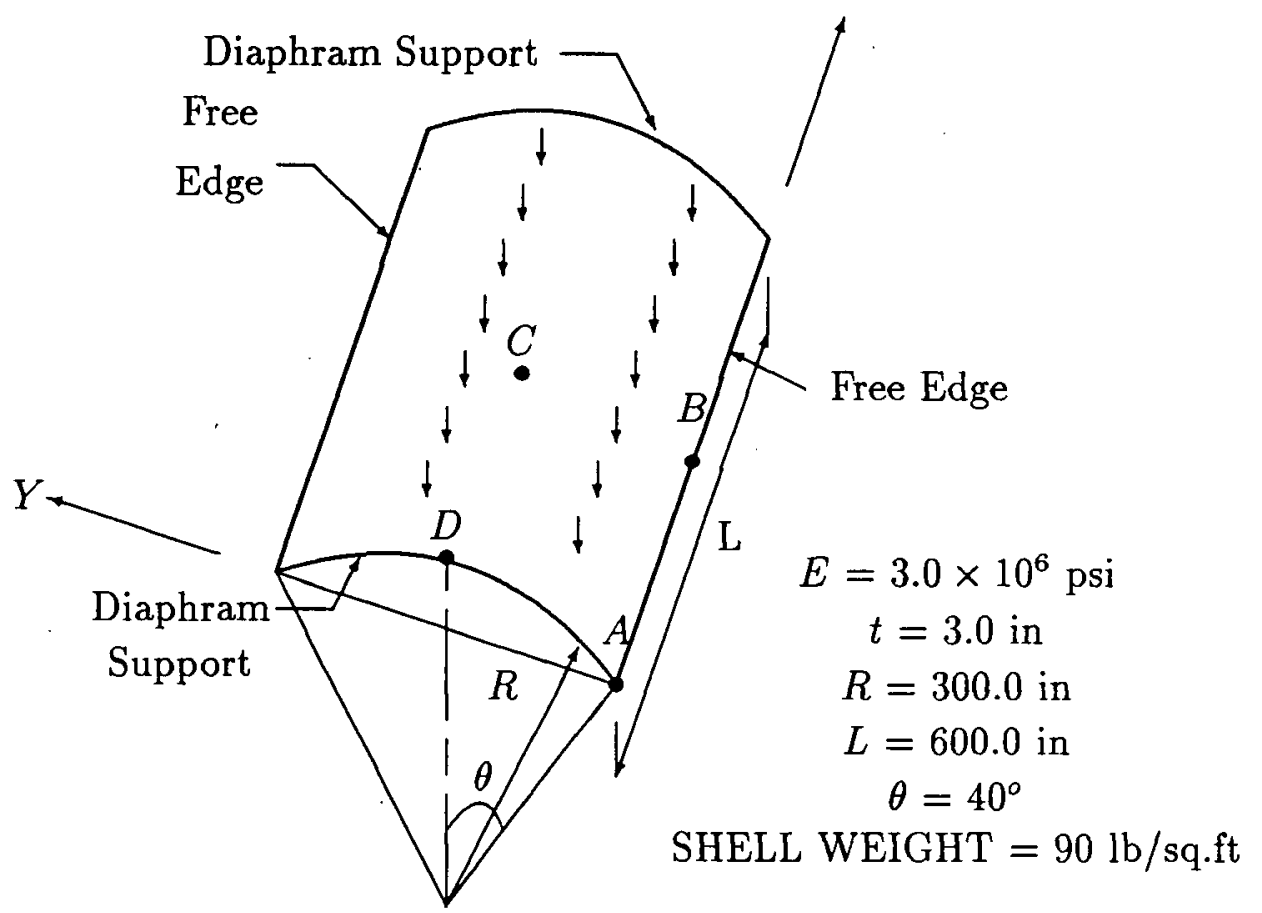

Figure 4.2: Shell-Roof problem 
The diaphram supports at the curved edges imply that the $v$ and $w$ displacements are restrained i.e. $v=w=0$ while the $u$ displacement is unrestrained i.e. $u \neq 0$. The in-plane force $N_{x}$ and the moment $M_{x}$ must also vanish along the diaphram supports.

In the flat plate approximation, the $u$-displacement at the curved edge has to be unconstrained. The choice of a cosine function satisfies this condition. The $v$ and $w$ components of the displacement are zero by virtue of the choice of a sine function as a mode shape. The in-plane force $N_{x}$ and the bending moment $M_{x}$ vanish at the diaphram supports for the linear analysis. However, by virtue of the choice of shape functions in the present analysis, the axial in-plane force is non-zero at the boundary for the large deflection case. Therefore, for the given shell-roof problem, the kinematic and the natural boundary conditions at the curved edges are satisfied exactly in the linear case but the natural boundary conditions are not completely satisfied in the non-linear case.

The loading on the shell is its own gravity load in the negative $Z$ direction of the global cartesian co-ordinate system. The load vector is of a pseudo-consistent formulation as described earlier.

A symmetric half of the shell is analyzed using up to 12 strips of equal widths i.e a 24 strip discretization for the whole shell. The results of the one mode finite-strip solution are compared with the analytical solutions of Scordelis and Lo [31], and with numerical procedures approximating the shell using triangular finite elements [32]. Table 4.2 summarizes the results of the linear elastic analysis.

The finite-strip results, using flat-plate elements do not converge monotonically in energy from below to the exact value. The central displacement of the free edge $\left(w_{B}\right)$ and the strain energy seem to be converging from above. 
Table 4.2: Linear Elastic Response of the Shell-Roof ProblemUsing Flat Strips

\begin{tabular}{|c|c|c|c|c|c|}
\hline $\begin{array}{c}\text { Number of } \\
\text { Finite Strips }\end{array}$ & $\begin{array}{c}10 u_{A} \\
(\mathrm{in})\end{array}$ & $\begin{array}{c}\mathrm{w}_{B} \\
(\mathrm{in})\end{array}$ & $\begin{array}{c}\mathrm{v}_{B} \\
(\mathrm{in})\end{array}$ & $\begin{array}{c}10 w_{C} \\
(\mathrm{in})\end{array}$ & $\begin{array}{c}\text { Strain Energy } \\
(\mathrm{K}-\mathrm{in})\end{array}$ \\
\hline 12 & -1.374 & -3.752 & 1.977 & 5.537 & 58.956 \\
18 & -1.4089 & -3.7054 & 1.955 & 5.473 & 58.18 \\
24 & -1.4214 & -3.6911 & 1.948 & 5.453 & 57.856 \\
Finite Element [32] \\
$\cdot \quad-1.5105$ & -3.69 & 1.955 & 5.226 & 58.65 \\
EXACT [31] & -1.513 & -3.703 & 1.963 & 5.249 & 58.828 \\
\hline
\end{tabular}

The use of a pseudo-consistent load vector in the finite strip analysis violates the finite-element criteria for a monotonic convergence to the exact solution from below. Also, it must also be noted that we are approximating a curved surface using flat plate strips. Refining the grid from 12 to 18 or 18 to 24 results in an entirely different structure, in other words, a. 24 strip grid does not necessarily include a 12 strip grid. Therefore, we cannot expect the exact solution to provide a upper bound for the strain energy. It is also seen that the strain-energy and the displacement components for a 24-strip approximation are lower than the corresponding exact values. In addition, since only a single mode is used for approximating the displacement components, we do not expect the finite strip solution to converge to the exact. Rather, it should converge to a one-mode analytical solution.

Even with a coarse grid of 12 finite strips, the one-mode solution is within $1.5 \%$ of the exact solution in the central vertical displacement of the free edge and within $0.5 \%$ in strain energy. Therefore, it is evident that a 12 -strip approximation can be used in the linear elastic analysis of the shell-roof problem subjected to gravity 
Table 4.3: Analysis of membrane and bending stresses in the Shell-Roof Problem Using Flat Plate strips

\begin{tabular}{|c|c|c|c|}
\hline $\begin{array}{c}\text { Number of } \\
\text { Finite Strips }\end{array}$ & $\begin{array}{c}N_{x B} \\
\text { Kips } / \text { in }\end{array}$ & $\begin{array}{c}M_{x C} \\
\text { Kips-in/in }\end{array}$ & $\begin{array}{c}\mathrm{M}_{y C} \\
\text { Kips-in/in }\end{array}$ \\
\hline 12 & 5.989 & -0.0809 & -2.00 \\
18 & 6.445 & -0.0917 & -2.025 \\
24 & 6.685 & -0.095 & -2.034 \\
Finite Element[31] & 6.5016 & -0.1284 & -2.0871 \\
EXACT [32] & 6.4124 & -0.0927 & -2.056 \\
\hline
\end{tabular}

load to obtain an accurate prediction of displacements and strain-energy.

Table 4.3 compares the membrane and bending stresses with the exact values obtained from the shallow shell theory. Both the membrane and the bending stresses compare very well with the exact values. The choice of continuously differentiable functions in the longitudinal direction apparently leads to a better convergence of stresses than earlier finite element analysis $[20],[32],[38]$.

Since a 12 strip model of a cylindrical shell gives a reasonable prediction of deflections, strain energy and stresses in the linear elastic case, all further analysis has been carried out using a grid of 12 finite strips. Fig. 4.3 shows the effect of large deformations on the response of the cylindrical shell when compared with the linear elastic response. Based on a finite-element analysis of the shell-roof problem, Bergan et al [33] concluded that incorporating geometric non-linearities leads to a relative stiffening in both displacements and strain-energy. However, in the present analysis, we observe that the central vertical displacement of the free edge is on the 
flexible side of the linear analysis up to a load level of $0.625 p s i$. Reversing the load leads to a stiffer solution for all values of the load. This result can be justified by arguing that when the load is applied in the negative $Z$ direction of the global coordinate system, membrane compression in the $Y$-direction leads to softening and hence, a more flexible solution. Conversely, when the load is reversed, membrane tension occurs in the $Y$-direction, which leads to stiffening.

The flat plate model of the cylindrical shell, although appropriate for the prediction of the linear elastic response of the cylindrical shell, does not provide a reliable tool when the effect of geometric non-linearities is incorporated. These limitations have also been highlighted by Flügge [3], wherein he observed that the membrane and bending theory of barrel vault roof using flat elements is subject to severe limitations. To overcome these limitations, another attempt at modelling the cylindrical shell using curved shell strips I was investigated. The results of the analysis are presented in the next section.

\subsection{Analysis of the Shell-Roof problem using Curved Shell strips I}

The same shell as was analyzed using flat plate strips is now modelled using Curved Shell strips I. As described in Section 3.6.2, the following aspects of the model need to be emphasized:

(a) The shape functions across the width of the shell strip for the in-plane displacements $u$ and $v$ are linear polynomials while those for the out-of-plane displacement $w$ are cubic. 
Chapter 4. Numerical Investigations

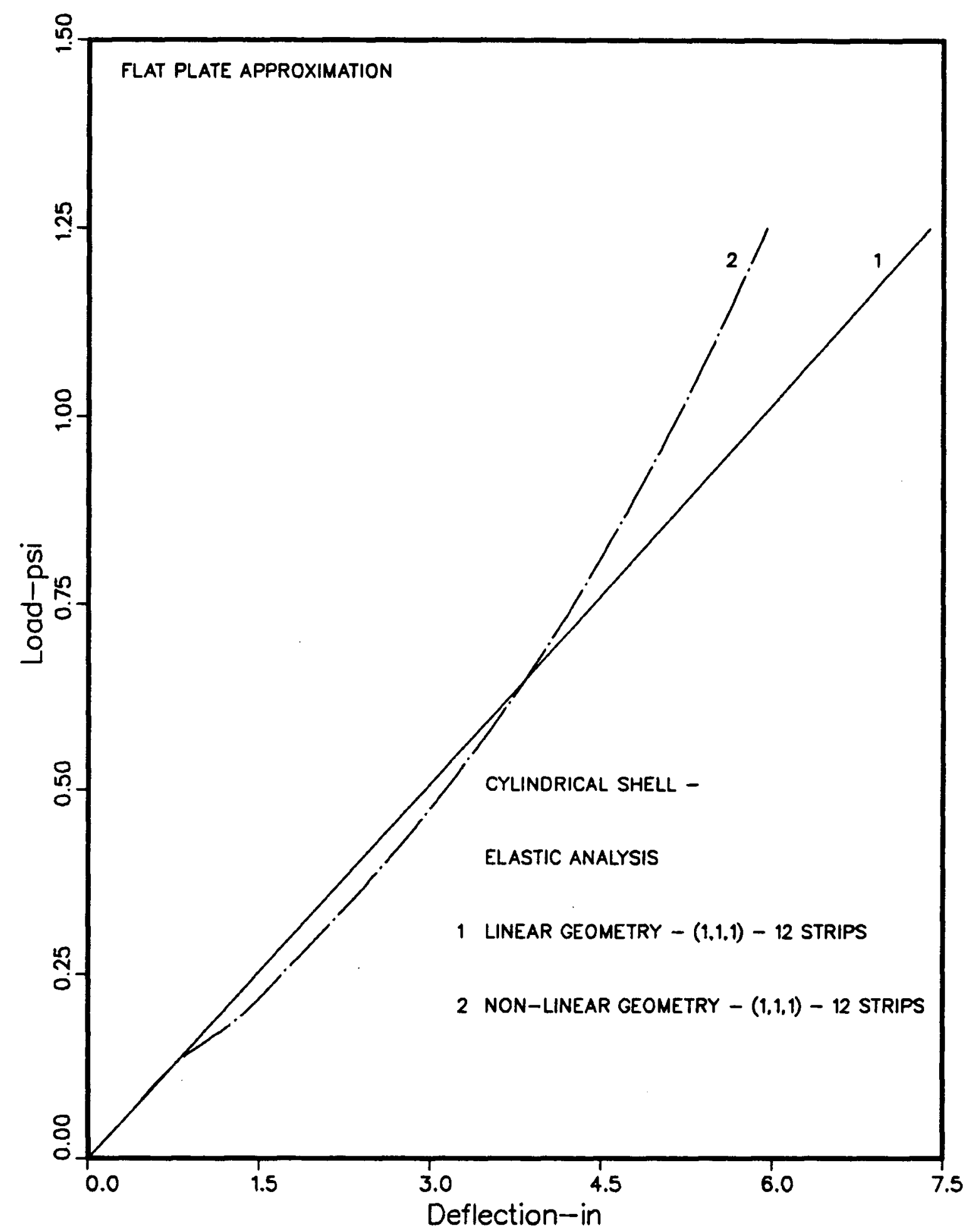

Figure 4.3: Vertical Displacement of the free edge 
(b) In this formulation, the strain-displacement functions for a cylindrical shell are used as opposed to the strain displacement relations of a plate in the Flat Plate formulation.

(c) The load vector generated for this formulation is consistent i.e. the gravity load on the shell is integrated exactly with the appropriate shape functions in both the longitudinal and the transverse directions.

One of the objectives for modelling the cylindrical shell using Curved shell strips I was to keep the number of degrees of freedom of the problem to be a minimum. Each finite strip in this formulation has 8 degrees of freedom as in the flat plate case. Only one mode is used to represent the displacement components $u, v$ and $w,(1,1,1)$ in our notation. Table 4.4 summarizes the results of the present linear elastic analysis and compares the displacement components and strain energy with the results of the analytical and finite-element analysis.

It is apparent that the finite strip solution using Curved Shell strips $I$ is extremely stiff. Comparing the strain energy and the vertical displacement of the free edge at its mid-span for a 24 strip approximation using flat plate strips, with the respective values obtained using Curved Shell strips I, we observe that the Curved Shell I model is $48 \%$ in error as compared with the exact strain energy and $63 \%$ stiffer in the displacement. Even when the grid is refined further, the convergence of strain energy and the displacement components to the exact solution is very slow.

Obviously, we don't expect the finite strip solution to converge to the exact solution as only a single mode is used to represent the displacement components in the longitudinal direction. However, we do expect it to converge to a one mode solution. Fig. 4.4 shows a plot of the relative error in strain energy, $\Delta U$, with 
Table 4.4: Linear Elastic Response of the Shell-Roof Problem Using Curved Shell Strips I

\begin{tabular}{|c|c|c|c|c|c|}
\hline $\begin{array}{c}\text { Number of } \\
\text { Finite Strips }\end{array}$ & $\begin{array}{c}10 u_{A} \\
(\mathrm{in})\end{array}$ & $\begin{array}{c}w_{B} \\
(\mathrm{in})\end{array}$ & $\begin{array}{c}10 v_{B} \\
(\mathrm{in})\end{array}$ & $\begin{array}{c}10 w_{C} \\
(\mathrm{in})\end{array}$ & $\begin{array}{c}\text { Strain Energy } \\
(\mathrm{K}-\mathrm{in})\end{array}$ \\
\hline 12 & -0.737 & -0.6213 & -2.75 & -2.023 & 20.71 \\
18 & -0.839 & -0.974 & -3.471 & -1.1316 & 25.32 \\
24 & -0.9267 & -1.356 & -4.183 & -0.196 & 29.78 \\
30 & -1.004 & -1.728 & -4.844 & 0.656 & 33.85 \\
36 & -1.071 & -2.064 & -5.426 & 1.392 & 37.85 \\
42 & -1.128 & -2.356 & -5.924 & 2.01 & 40.52 \\
72 & -1.294 & -3.25 & -7.42 & 3.18 & 49.64 \\
78 & -1.313 & -3.35 & -7.59 & 4.02 & 50.65 \\
Finite Element [32] & -1.5105 & -4.08388 & -8.73995 & 5.2258 & 58.65 \\
EXACT [31] & -1.513 & -4.099 & -8.761 & 5.249 & 58.828 \\
\hline
\end{tabular}

the number of finite strips used in the analysis, N. The order of convergence of the strain energy lies between $O(N)$ and $O\left(N^{-2}\right)$, which is rather slow.

The order of accuracy analysis carried out in Section 3.4 reveals that the choice of linear polynomials to represent the in-plane displacements in the transverse direction does not predict a consistent rate of convergence. The order of convergence expected due to the presence of terms like $v_{y}^{2}$ and $u_{y}^{2}$ is of $O(N)$ while the corresponding order due to terms in the strain energy expression like $w_{y y}^{2}$ is of $O\left(N^{-4}\right)$. Thus, it is not very surprising that the convergence of strain-energy to the exact 


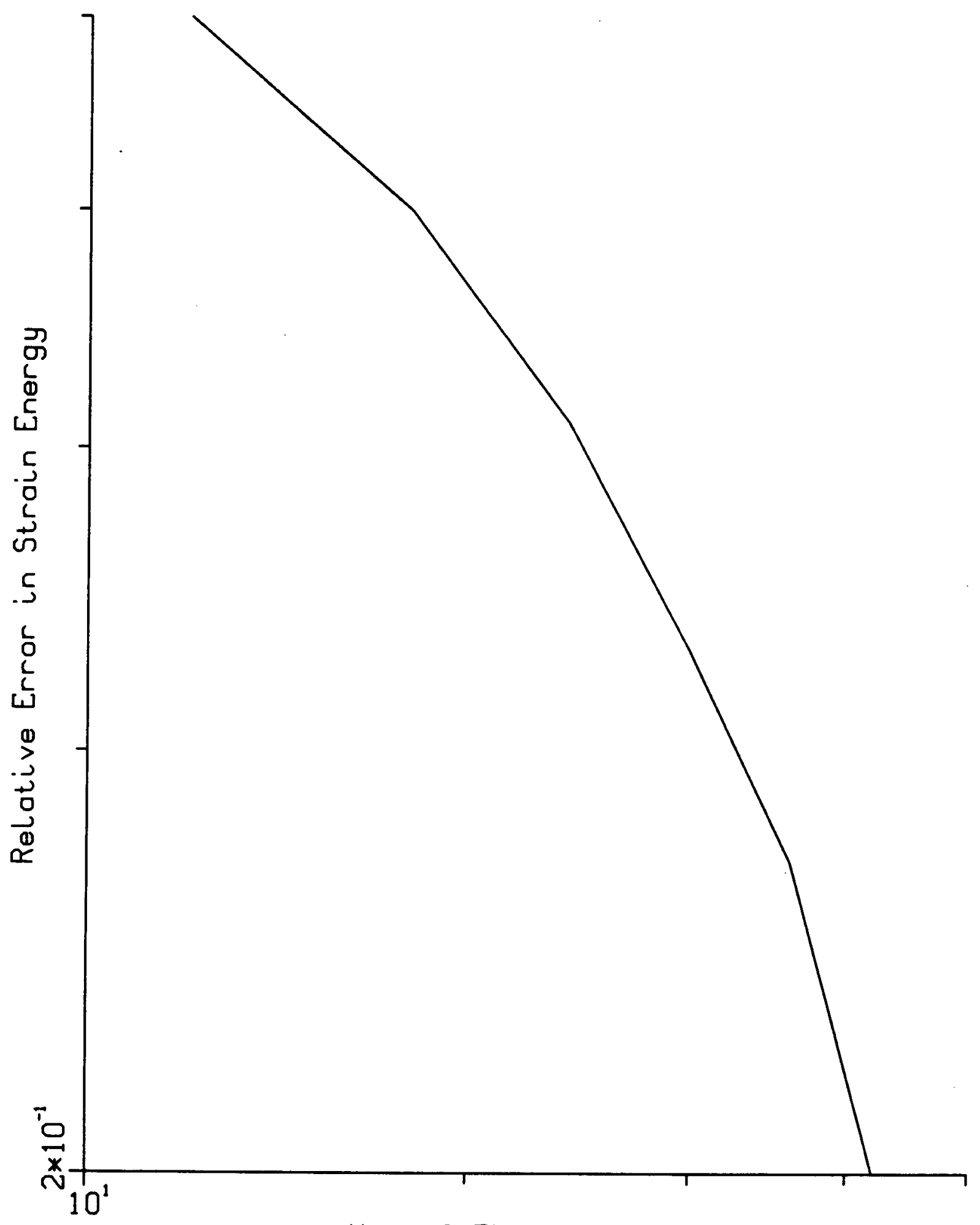

No. of Finite Strips

Figure 4.4: Relative Error in Strain Energy using Curved Shell strips I 
value lies between $O(N)$ and $O\left(N^{-2}\right)$. The above analysis conclusively proves that the Curved Shell I model is inadequate from a practical standpoint to represent the cylindrical shell. By upgrading the shape functions for the in-plane displacements from linear to quadratic, the order of accuracy analysis in Section 3.4 predicts a consistent order of convergence.

\subsection{Analysis of the Shell-Roof Problem using Curved Shell strips II}

The same shell as was analyzed using flat plate and Curved Shell strips I is now modelled using Curved Shell strips II. Some salient features of this formulation are:

(a) The shape functions are upgraded from linear to quadratic polynomials for the in-plane displacement components in the transverse direction requiring the introduction of an internal nodal line.

(b) The numerical integration across the width of each finite strip is carried out at 3 Gauss points so as to integrate a fourth order polynomial exactly.

(c) The load vector for gravity load in the negative $Z$ direction is integrated in closed form to obtain a consistent load vector.

Table 4.5 summarizes the results of the linear elastic response of the Shell-Roof problem subjected to gravity load. The analysis employs a single mode for each of the displacement components: $u, v$ and $w$.

The radial displacement at the mid-span of the free edge for a 24-strip approximation is within $0.3 \%$ of the exact, while the radial displacement of the crown 
Table 4.5: Linear Elastic Response of the Shell-Roof Problem Using Curved Shell Strips II

\begin{tabular}{|c|c|c|c|c|c|}
\hline $\begin{array}{c}\text { Number of } \\
\text { Finite Strips }\end{array}$ & $\begin{array}{c}10 u_{A} \\
(\mathrm{in})\end{array}$ & $\begin{array}{c}w_{B} \\
(\mathrm{in})\end{array}$ & $\begin{array}{c}10 v_{B} \\
(\mathrm{in})\end{array}$ & $\begin{array}{c}10 w_{C} \\
(\mathrm{in})\end{array}$ & $\begin{array}{c}\text { Strain Energy } \\
(\mathrm{K}-\mathrm{in})\end{array}$ \\
\hline 6 & -1.306 & -3.4 & -7.577 & 3.88 & 47.84 \\
12 & -1.435 & -4.03 & -8.708 & 5.323 & 54.34 \\
24 & -1.446 & -4.087 & -8.798 & 5.439 & 54.81 \\
Finite Element [32] & -1.510 & -4.08388 & -8.73995 & 5.2258 & 58.65 \\
EXACT [31] & -1.513 & -4.099 & -8.761 & 5.249 & 58.828 \\
\hline
\end{tabular}

is $3.5 \%$ more flexible than the corresponding exact solution. The strain energy is $6.8 \%$ in error as compared with the exact solution but is converging monotonically from below. The fact that the radial displacement at the mid-span of the crown is more flexible than the exact value while the strain energy is on the lower side of the exact value is a result of using a one mode approximation and was also observed in Abayakoon's work [24] on unstiffened plates. The error in strain energy might seem to be large as compared with the exact value, but it must be noted that only one bending mode is employed in the present analysis. The cost of computation is minimal compared to other finite-element procedures [20],[32] and an accurate prediction of displacements and strain energy is still obtained.

Fig. 4.5 represents the plot of the relative error in strain energy as the finite strip grid is refined. From the plot it can be inferred that the strain energy convergent rate is of the order of $N^{-4}$. Therefore, it can be concluded that the curved shell strip II models the geometry of the cylindrical panel as well as its linear elastic 
behavior exceptionally well.

The membrane and bending stresses in the cylindrical shell have been computed at several points. Table 4.6 summarizes the results of the present analysis. Comparisons with the finite element procedures and analytical results is also presented.

Table 4.6: Analysis of membrane and bending stresses in the Shell-Roof Problem Using Curved Shell strips II

\begin{tabular}{|c|c|c|c|}
\hline $\begin{array}{c}\text { Number of } \\
\text { Finite Strips }\end{array}$ & $\begin{array}{c}N_{x B} \\
\text { Kips /in }\end{array}$ & $\begin{array}{c}M_{x C} \\
\text { Kips-in/in }\end{array}$ & $\begin{array}{c}\mathrm{M}_{y C} \\
\text { Kips-in/in }\end{array}$ \\
\hline 6 & 4.245 & 0.161 & -1.308 \\
12 & 5.23 & -0.072 & -2.008 \\
24 & 5.65 & -0.094 & -2.111 \\
Finite Element [31] & 6.5016 & -0.1284 & -2.087 \\
EXACT [32] & 6.412 & -0.0927 & -2.056 \\
\hline
\end{tabular}

From the results it is evident that both the membrane and bending stresses compare very well with the respective analytical results and are not much different from the results of the flat plate representation.

The numerical investigations of the shell-roof problem have revealed that the Curved Shell II model is superior to the Curved Shell I model. It can also be seen that the displacements and stresses are predicted with good accuracy using a flat plate formulation. However, a monotonic convergence in strain energy is not achieved. In the Curved Shell II formulation, the strain energy is converging monotonically to a one mode solution. All requirements of the classical finite element theory are also satisfied in this formulation. Even with a single mode in the 


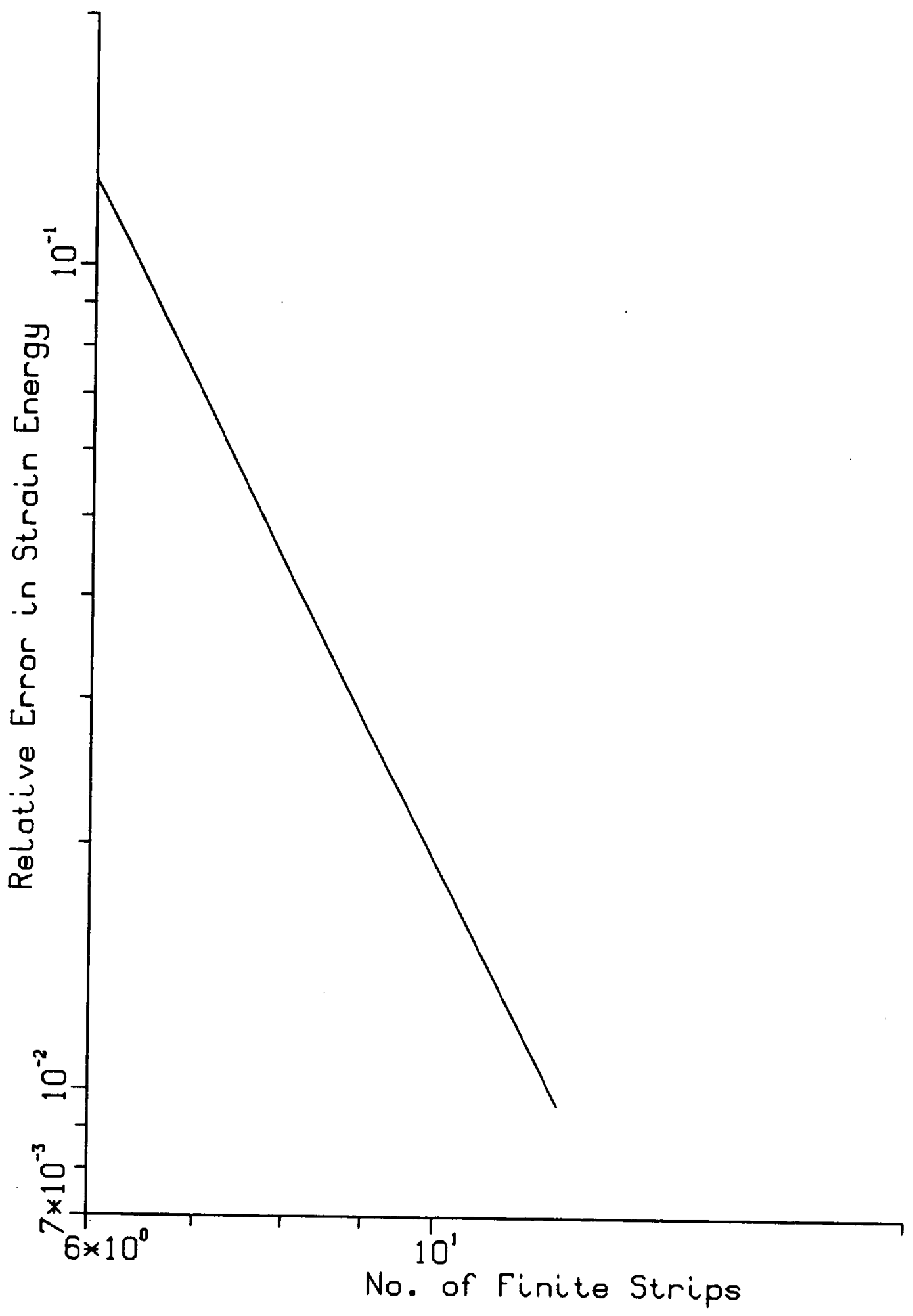

Figure 4.5: Relative Error in Strain Energy Using Curved Shell strips II 
longitudinal direction, the displacements, stresses and strain-energy have been predicted reasonably accurately. The accuracy of the one-mode solution in modelling an axisymmetric cylindrical shell subjected to a uniform internal pressure using Curved Shell strips II is now examined.

\subsection{Analysis of a Cylindrical Shell Loaded Symmetrically with Respect to Its Axis}

The linear elastic response of a simply supported cylindrical shell subjected to uniform pressure is investigated to test the applicability and accuracy of the finite strip formulation. The geometry of the problem is depicted in Fig. 4.6 .

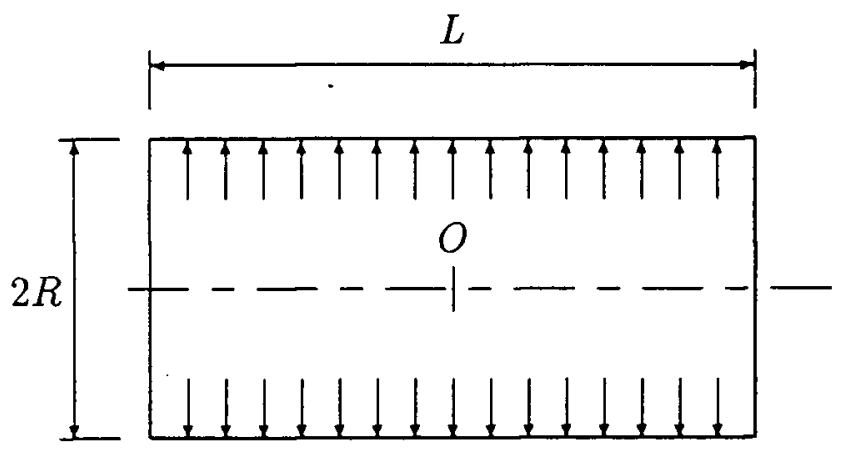

Figure 4.6: Axisymmetric Cylindrical Shell

The boundaries of the shell are simply supported i.e. the deflection $w$ and the 
moment $M_{x}$ vanish at the supported ends. Further from symmetry, the membrane shearing forces $N_{x y}=N_{y x}$ and the twisting moments $M_{x y}=M_{y x}$ vanish in this case, and the circumferential membrane forces $N_{y}$ and the bending moments $M_{y}$ are functions only of $x$.

The analytical solution has been obtained by solving the equations of equilibrium and is discussed in detail in Timoshenko [4]. The origin of co-ordinates is taken at the middle of the cylinder. The final expression for the normal displacement, $w$, for the general case is given by:

$$
\begin{aligned}
w= & \frac{-p L^{4}}{64 D \gamma^{4}}\left(1-\frac{2 \sin \gamma \sinh \gamma}{\cos 2 \gamma+\cosh 2 \gamma} \sin \psi x \sinh \psi x\right. \\
& \left.-\frac{2 \cos \gamma \cosh \gamma}{\cos 2 \gamma+\cosh 2 \gamma} \cos \psi x \cosh \psi x\right)
\end{aligned}
$$

where $\psi^{4}=\frac{E t}{4 R^{2} D}=\frac{3\left(1-\nu^{2}\right)}{R^{2} t^{2}}$

and $\gamma$ is a non-dimensional quantity dependent on the shell geometry and is given by:

$$
\gamma=\left(\frac{\left[3\left(1-\nu^{2}\right)\right]^{\frac{1}{4}}}{2}\right) \frac{L}{\sqrt{R t}}
$$

At the middle of the shell i.e. $x=0$, the normal deflection is given by:

$$
(w)_{x=0}=\frac{-p L^{4}}{64 D \gamma^{4}}\left(1-\frac{2 \cos \gamma \cosh \gamma}{\cos 2 \gamma+\cosh 2 \gamma}\right)
$$

The expressions for the moment $M_{x}$ and the strain energy $U$ can easily be obtained by differentiation and integration of the expression for deflections respectively and subsequent substitution in the appropriate formula. The expressions are 
given by:

$$
\begin{aligned}
M_{x}= & -\frac{p L^{2}}{4 \gamma^{2}}\left(\frac{\sin \gamma \sinh \gamma}{\cos 2 \gamma+\cosh 2 \gamma} \cos \psi x \cosh \psi x\right. \\
& \left.-\frac{\cos \gamma \cosh \gamma}{\cos 2 \gamma+\cosh 2 \gamma} \sin \psi x \sinh \psi x\right)
\end{aligned}
$$

and,

$$
\begin{aligned}
U= & \frac{-p^{2} L^{4} b}{128 D \gamma^{4}}\left\{L-\frac{2 \sin \gamma \sinh \gamma}{\cos 2 \gamma+\cosh 2 \gamma}\left[\left(\frac{\gamma^{3}}{1+\gamma^{4}}\right) \sin \frac{\psi L}{2} \cosh \frac{\psi L}{2}\right.\right. \\
& \left.+\left(\frac{\gamma}{1+\gamma^{4}}\right) \cos \frac{\psi L}{2} \sinh \frac{\psi L}{2}\right] \\
& -\frac{2 \cos \gamma \cosh \gamma}{\cos 2 \gamma+\cosh 2 \gamma}\left[\left(\frac{\gamma^{3}}{1+\gamma^{4}}\right) \cos \frac{\psi L}{2} \sinh \frac{\psi L}{2}\right. \\
& \left.\left.-\left(\frac{\gamma}{1+\gamma^{4}}\right) \sin \frac{\psi L}{2} \cosh \frac{\psi L}{2}\right]\right\}
\end{aligned}
$$

The finite strip analysis is carried out using a one strip discretization to model a quarter of the cylindrical shell. The analysis is carried out using a single mode for each of the displacement components $u, v$ and $w$ i.e. $(1,1,1)$ in our notation. The shell is subjected to uniform internal pressure. The shell is analyzed for six values of the parameter $R t / L^{2}$ i.e. $0.004,0.01,0.02,0.04,0.0625$ and 0.11 . Typical numerical results are presented in Table 4.7 for the radial displacement at the center and the strain energy for a linear elastic analysis.

The deflection profiles of the cylindrical shell are plotted in Fig. 4.7. It is obvious that as the parameter $R t / L^{2}$ increases, the one-mode finite strip solution compares very well with the analytical solution. However, the smaller the value of the nondimensional parameter, $R t / L^{2}$, the greater is the discrepancy between the finite 
Table 4.7: Linear Elastic Response of an Axisymmetric Cylindrical Shell Using Curved Shell Strips II

$$
\begin{aligned}
E & =3.0 \times 10^{6} \mathrm{psi} \\
\nu & =0.3 \\
p & =1.5 \mathrm{psi}
\end{aligned}
$$

\begin{tabular}{|c|c|c|c|c|c|}
\hline$R t / L^{2}$ & $\begin{array}{c}\text { Effective Boundary } \\
\text { Layer Width } \\
\text { (in) }\end{array}$ & $\begin{array}{c}\text { Radial Displacement } \\
\text { (in) }\end{array}$ & \multicolumn{2}{|c|}{$\begin{array}{c}\text { Strain Energy } \\
\text { (K-in) }\end{array}$} \\
\cline { 3 - 6 } & Finite Strip & Exact & Finite Strip & Exact \\
\hline 0.004 & $L / 15$ & 0.0190 & 0.0150 & 1.933 & 2.386 \\
0.01 & $L / 10$ & 0.0190 & 0.0149 & 1.288 & 1.590 \\
0.02 & $L / 7$ & 0.0190 & 0.0150 & 0.908 & 1.125 \\
0.04 & $L / 5$ & 0.0188 & 0.0162 & 0.635 & 0.795 \\
0.0625 & $L / 4$ & 0.0184 & 0.0169 & 0.498 & 0.636 \\
0.11 & $L / 3$ & 0.0172 & 0.0166 & 0.348 & 0.477 \\
\hline
\end{tabular}

strip and the analytical solution. This can be explained by a closer examination of the parameter, $R t / L^{2}$. The investigations of the spherical cap problem subjected to uniform pressure and freely supported boundaries by Cowper et al [32] revealed that the parameter $R t / L^{2}$ corresponds to an effective boundary layer width in a shell which can algebraically be written as:

$$
W_{\text {eff }}=\sqrt{R t}
$$

It was also found that as the value of the parameter approached zero the spherical shell exhibited membrane behavior. In the axisymmetric cylindrical shell problem, it is apparent that as the parameter $R t / L^{2}$ decreases, the effective boundary layer 
width decreases and the behavior of the shell away from the edges approaches that of a shell with free edges. For such a case, the internal pressure, p, produces a hoop stress $\sigma_{\circ}$ given by

$$
\sigma_{o}=\frac{p R}{t}
$$

and a resulting radial deflection, $\delta$, given by

$$
\begin{aligned}
\delta & =\frac{R \sigma_{0}}{E} \\
& =\frac{p R^{2}}{E t}
\end{aligned}
$$

However, when the parameter $R t / L^{2}$ increases, i.e. when $\gamma$ is very small, the midspan deflection approaches a value of $5 \gamma^{2} / 6$ which approximates the solution of a uniformly loaded simply supported beam.

From the analysis, it can therefore be concluded that the one mode solution is capable of representing the axisymmetric solution only for cylindrical shells with large effective boundary layer widths. From the deflection profiles along the span, it can be seen that the finite strip solution provides a good approximation to the analytical solution for a value of the parameter $R t / L^{2}$ not less than 0.04 . This corresponds to an effective boundary layer width of $L / 5$. Beyond this value i.e. for a smaller effective boundary layer width, it is necessary to use additional bending modes to model the axisymmetric solution. In the present analysis, the first bending mode is a half sine wave. By superposing higher modes like $\sin 3 \pi \xi$, $\sin 5 \pi \xi$ etc., the deflection profile will tend to flatten out both at the mid-span and close to the boundaries which would be a much better approximation to the analytical solution. A plot of moment distribution along the span of the cylindrical shell in Fig. 4.8 seems to corroborate the earlier comments on the accuracy of the one mode solution to the analysis of the axisymmetric cylindrical shell problem. 

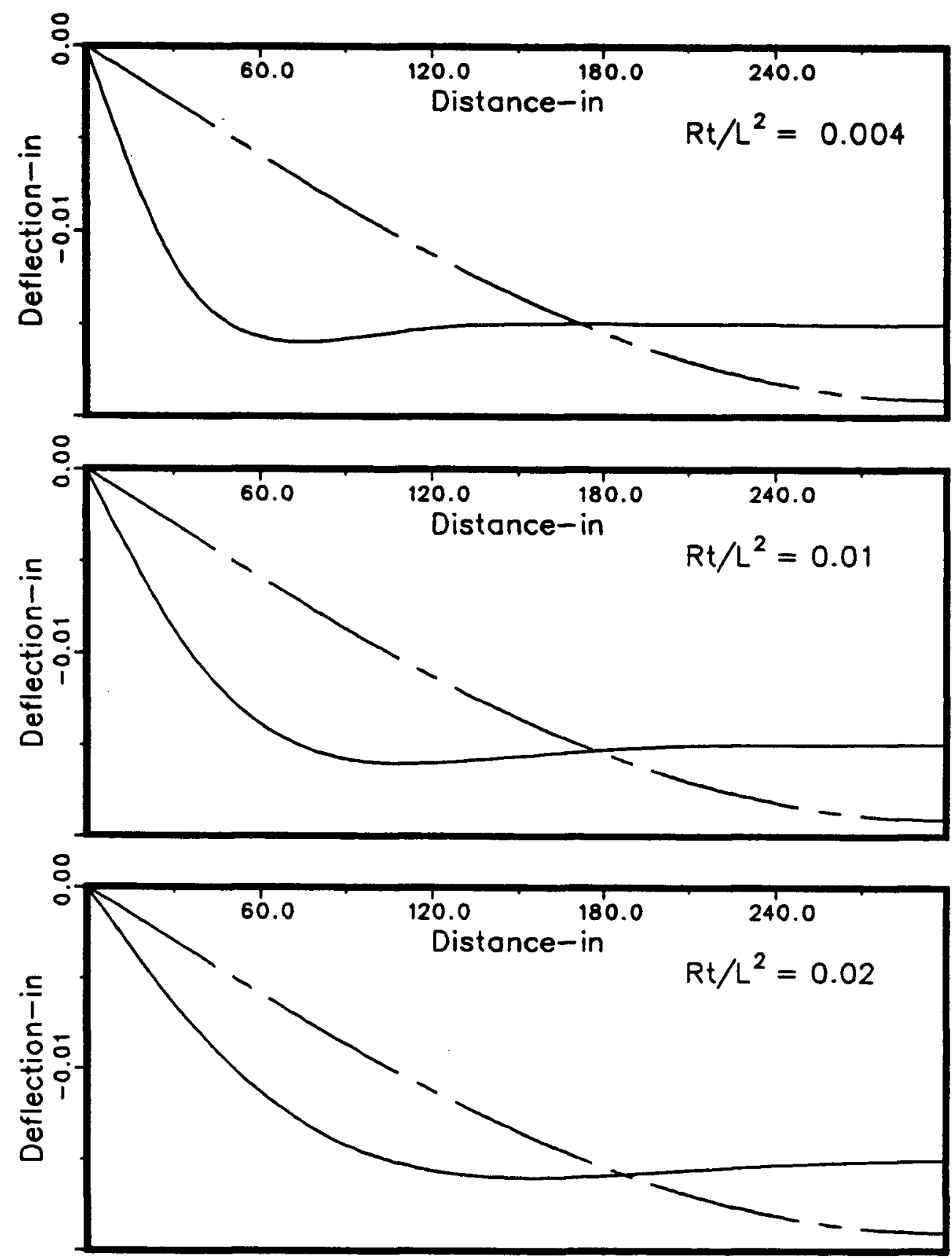

- FINITE STRIP - $(1,1,1)$

ANALYTICAL SOLUTION

Figure 4.7: Radial Displacement 

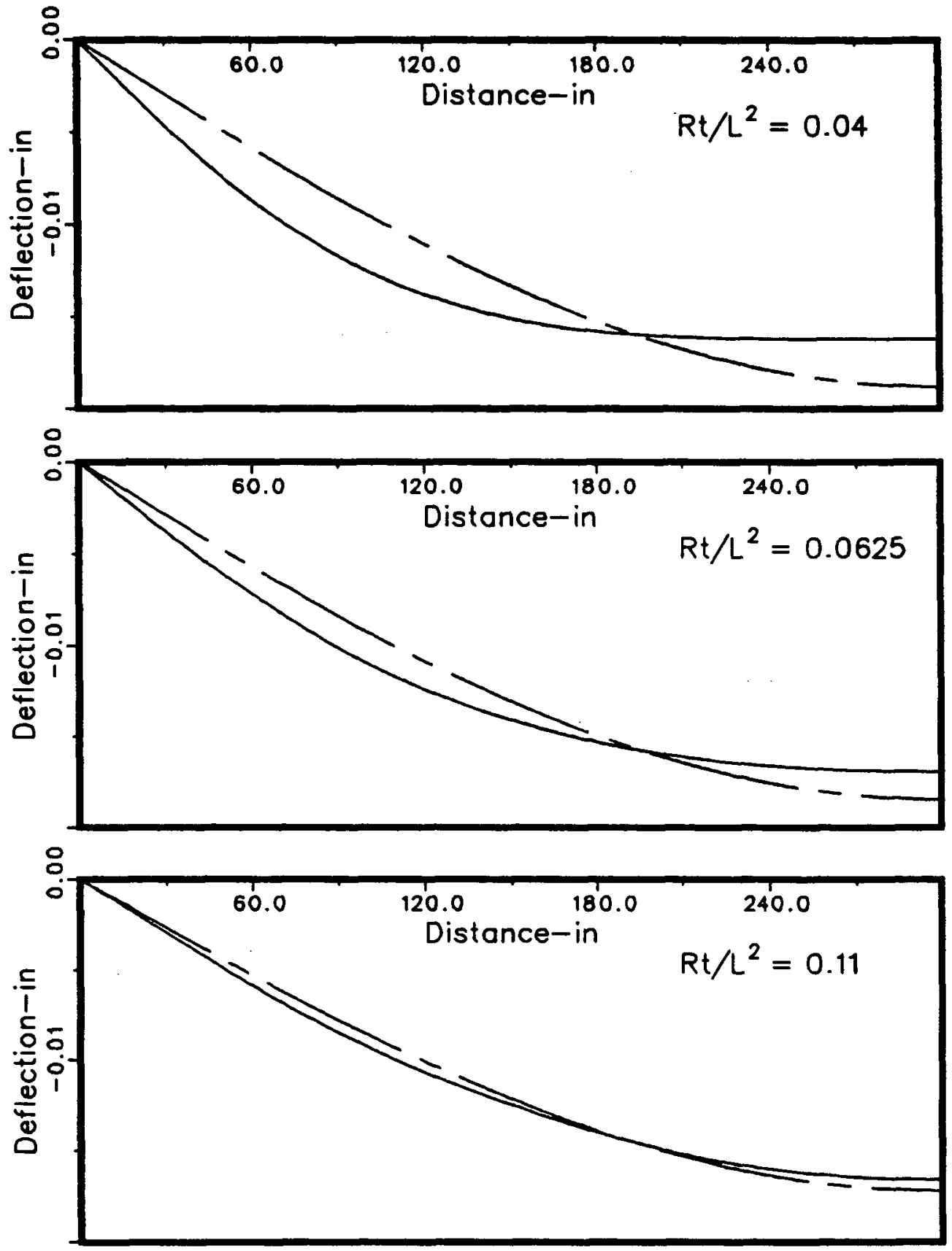

- FINITE STRIP - $(1,1,1)$

ANALYTICAL SOLUTION

Figure 4.7: Radial Displacement 


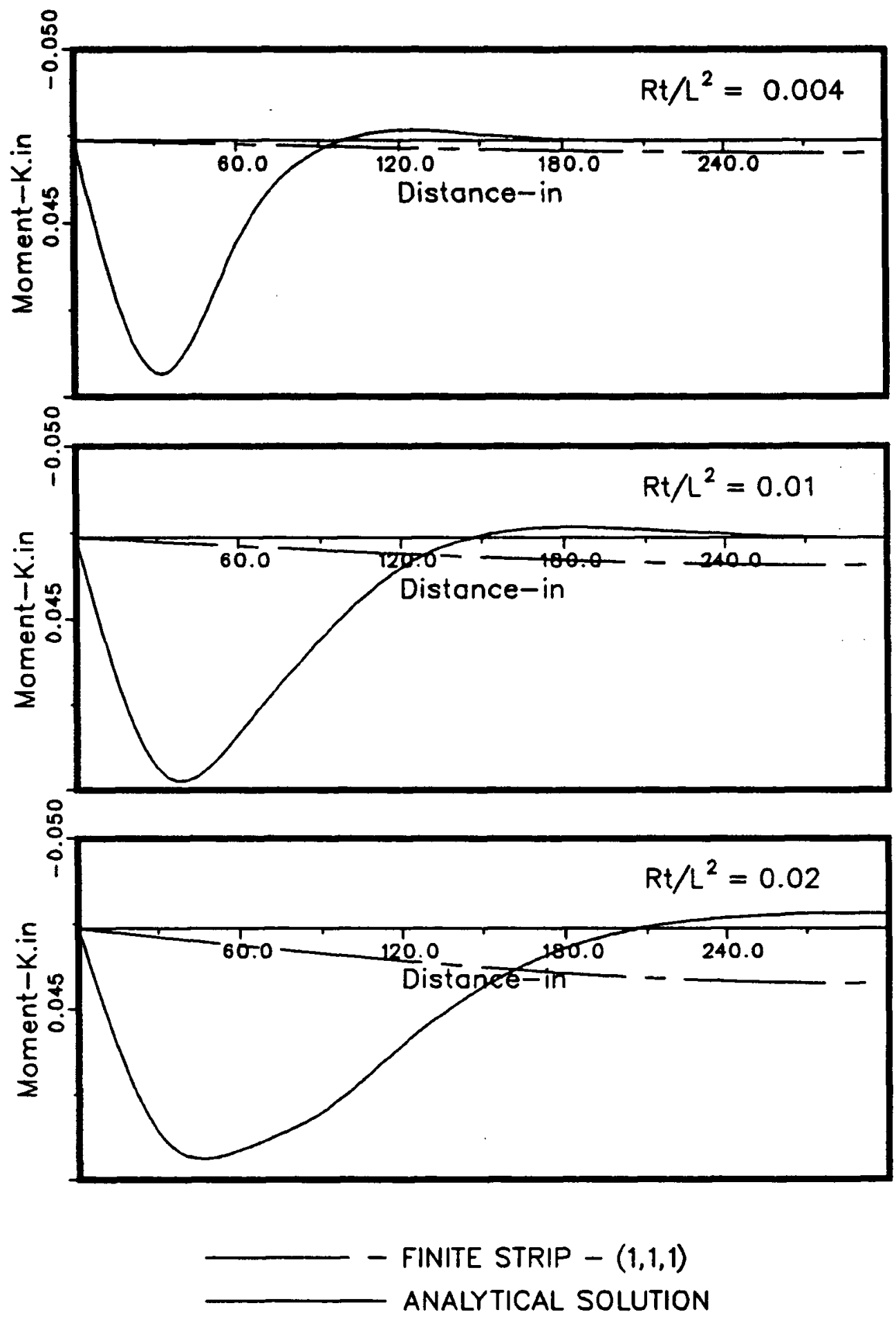

Figure 4.8: Moment Distribution 

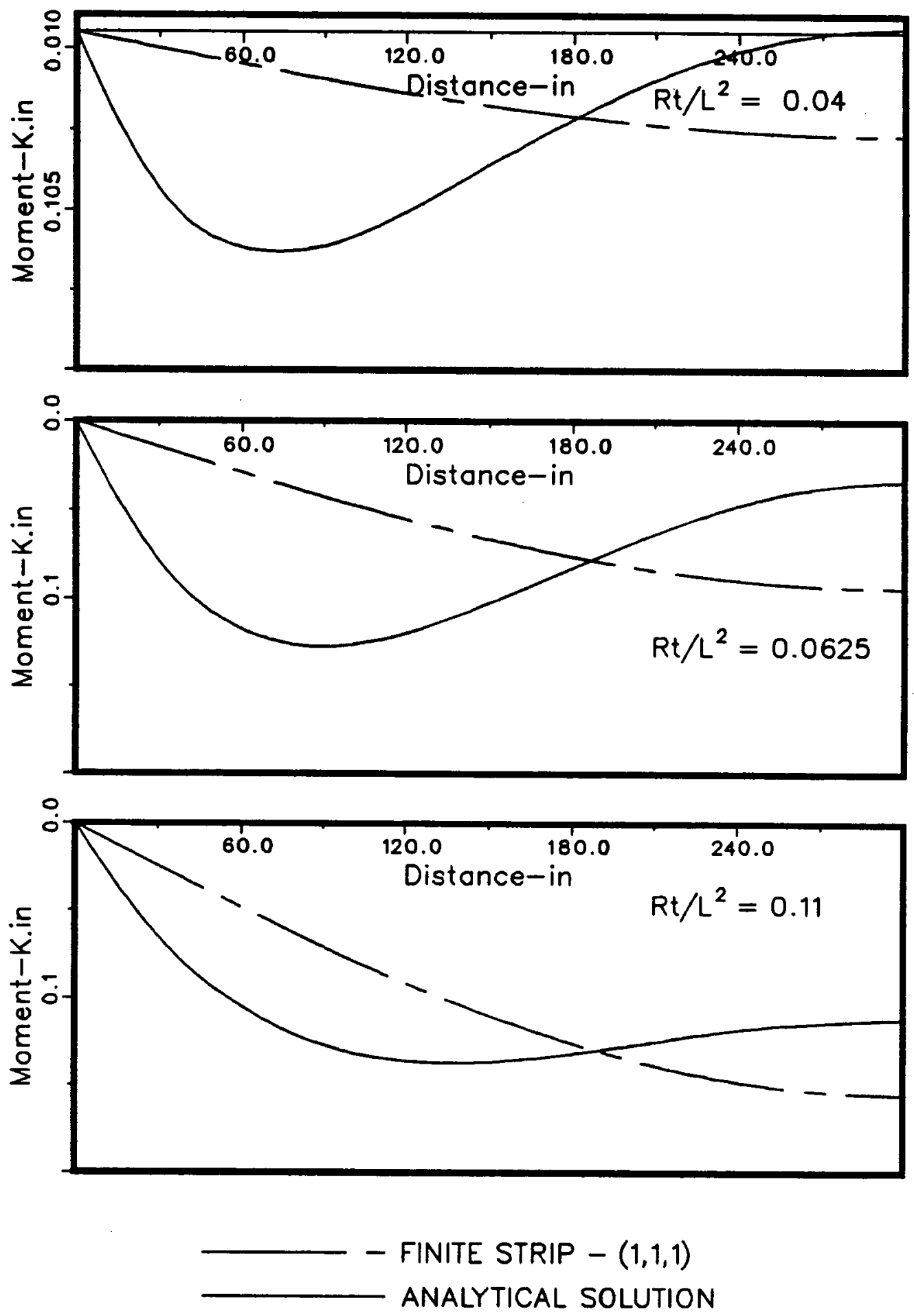

Figure 4.8: Moment Distribution 
Thus in axisymmetric problems, the one-mode solution is limited in its application to those cases with an effective boundary layer width exceeding $L / 5$ and even here the moments are not in good agreement. As the effective boundary layer width decreases, the limitations of the one-mode solution are obvious. These limitations can be overcome by using additional bending modes.

\subsection{Non-Linear Analysis of the Shell-Roof}

\section{Problem}

\subsubsection{Linear Elastic-Plastic Analysis}

The shell-roof problem is now analyzed by assuming an elastic-perfectly plastic maierial. The material behavior of the shell is determined by the following parameters:

Elastic modulus $\quad \mathrm{E}=21000 \times 10^{3} \quad \mathrm{kN} / \mathrm{m}^{2}$

Plastic modulus $\quad \mathrm{E}_{T}=0.0 \quad k N / m^{2}$

Poisson's Ratio $\quad \nu=0.0$

Yield Stress $\sigma_{0}=4.1 \times 10^{3} \quad k N / m^{2}$

Maximum Load Level $\quad \mathrm{p}=-3.0 \quad k N / \mathrm{m}^{2}$

where $E_{T}$ is the slope of the plastic segment of the bi-linear stress-strain curve. A 12-strip discretization for the symmetric half of the cylindrical shell using Curved Shell strips II is used in the investigation.

The results for the vertical displacement of the free edge are plotted in Fig. 4.9, and are compared with the results of the linear elastic-plastic analysis of the shell using degenerate iso-parametric shell elements carried out by Owen et al [21] who 
discretized a quarter of the shell using 6 such shell elements.

Initial yield takes place at a load level of $1.2 \mathrm{kN} / \mathrm{m}^{2}$ but the displacements remain quite linear up to a load of $2 \mathrm{kN} / \mathrm{m}^{2}$. The mid-span deflections by the finite strip method are on the flexible side of the Owen et al [21] analysis for most of the load range but are nearly identical at high load levels.

Fig. 4.10 shows a plot of load versus work done by the load for the finite strip analysis for varying number of strips. There appears to be a monotonic convergence of work done by the load with mesh refinement, and the small difference between the 12 and 24 strip results verifies that 12 strips are adequate for this problem.

\subsubsection{Large Deflection Elastic Analysis}

The results of the large deflection elastic analysis of the shell-roof problem are now compared. The finite strip solution is obtained by using 12 strips in the symmetric half of the shell. The results from a finite element analysis utilizing a 3 (axial) $\times 4$ (circumferential) mesh of rectangular shell elements for a quarter of the shell by Crisfield [13] are plotted for comparison.

Fig. 4.11 shows the vertical displacement at the center of the free edge for different methods of analysis. At lower loads and for linear solutions, good agreement is obtained between the one-mode finite strip and the two finite element analyses. The non-linear finite strip solution is, however, on the stiff side of the finite element analyses.

Abayakoon [24] found that for problems where the non-linear geometrical terms became important a second $u$ mode was required to model the uniform axial membrane force in the $X$-direction. By adding a second $u$ mode, $(1,1,1)+\left(3,,_{--}\right)$in our 


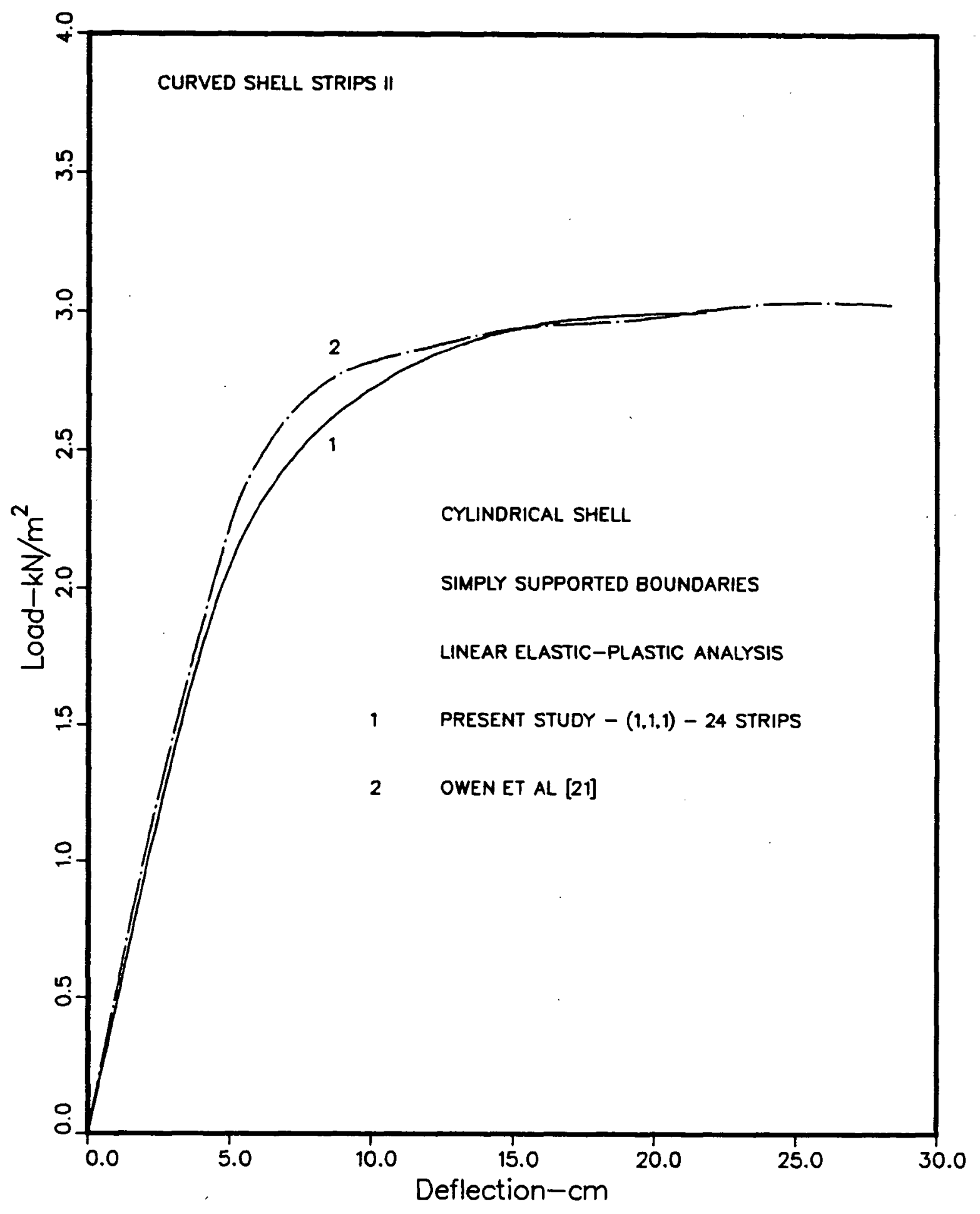

Figure 4.9: Vertical Displacement of the free edge 


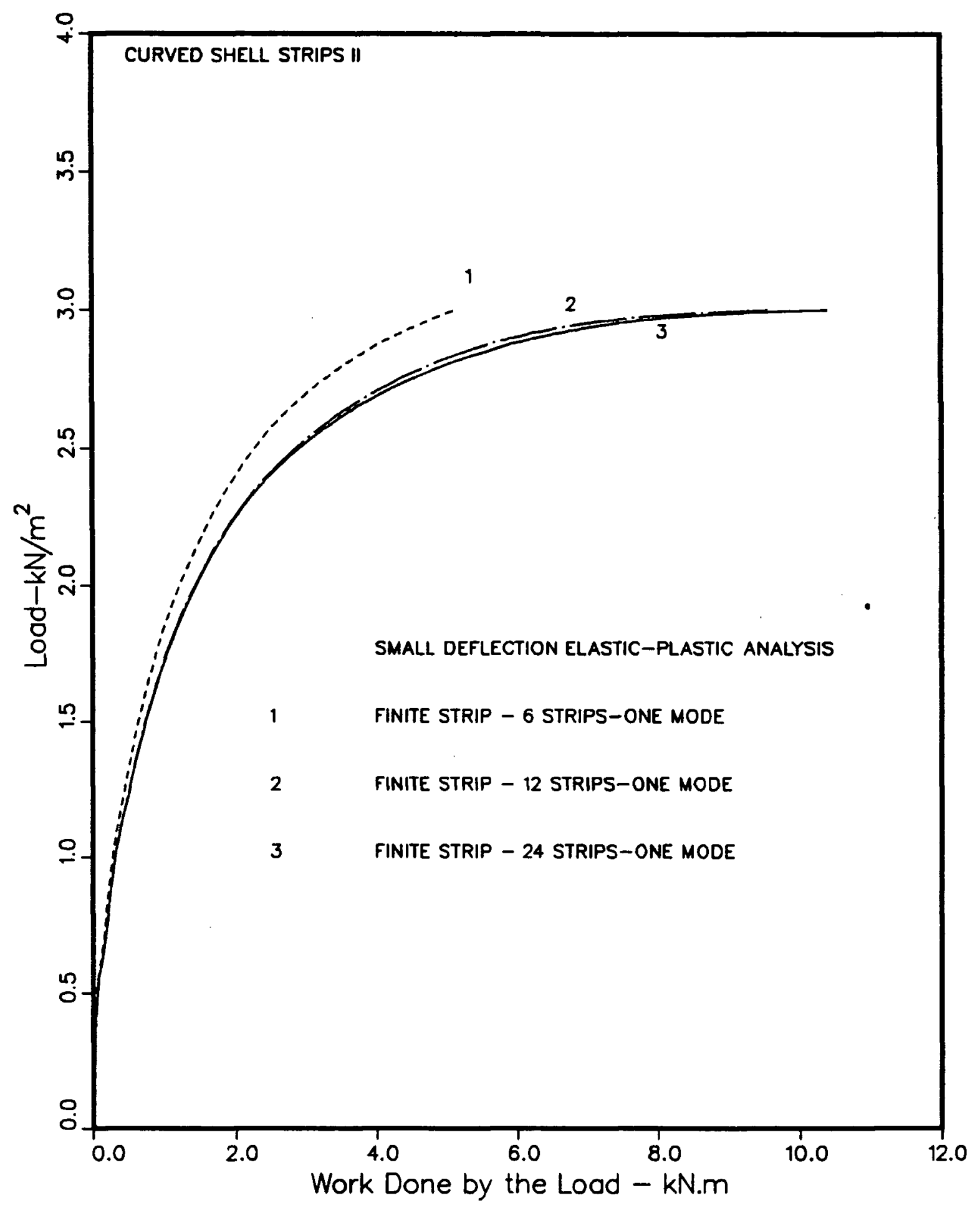

Figure 4.10: Variation of Work Done by the Load for the Shell-Roof Problem 


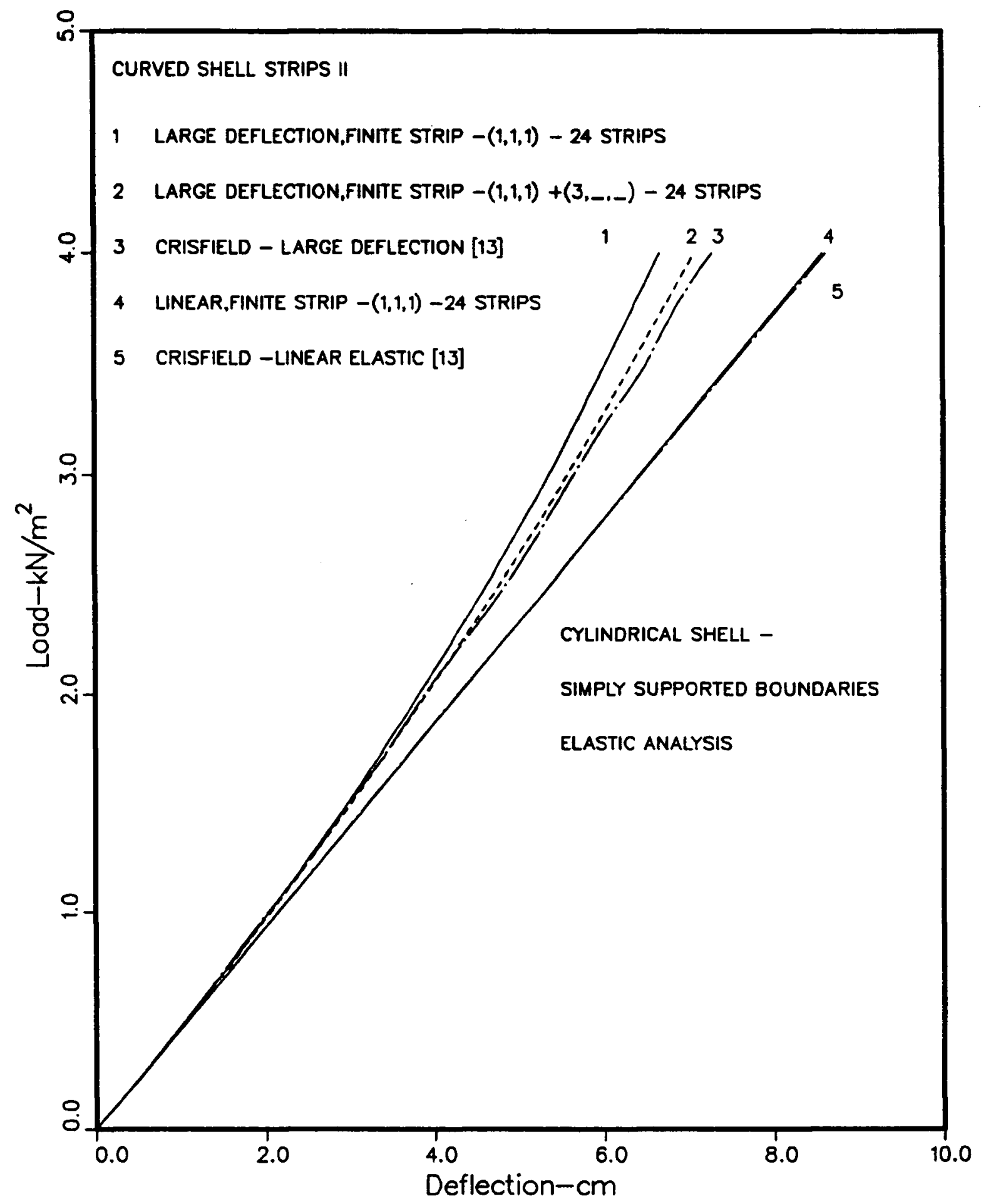

Figure 4.11: Vertical Displacement of the free edge 
notation, the displacement at higher load levels increased giving good agreement between the results of the finite strip and the finite element solution.

The variation of strain energy as the finite strip grid is refined is depicted in Fig. 4.12 . Monotonic convergence is apparent and again it is clear that 12 strips are sufficient for this problem.

\subsubsection{Large Deflection Elastic-Plastic Analysis}

The effect of incorporating the geometric and material non-linearities on the vertical deflection of the free edge in the finite strip analysis of the shell-roof problem is depicted in Fig. 4.13. The results from a finite element analysis utilizing a $4 \times 4$ mesh of curved degenerate isoparametric shell elements for a quarter of the shell by Bergan et al [33] and using curved degenerate isoparametric shell elements by Owen et al [21] have also been plotted. The results of Heppler [41], who used bi-cubic displacement trial functions in his finite element formulation for shell elements, are also shown.

At lower load levels, the results of the one-mode finite strip solution compare very well with all the finite element results but is very stiff at higher load levels. It is clear that adding a second $u$ mode makes some improvement but the results are still not satisfactory. What is surprising is the apparent stiffness of the finite strip analysis at high load levels, especially in view of good agreement for the separate analysis of elastic-nonlinear geometry and plastic-linear geometry.

The variation of the work done by the load with grid refinement is depicted in Fig. 4.14 . 


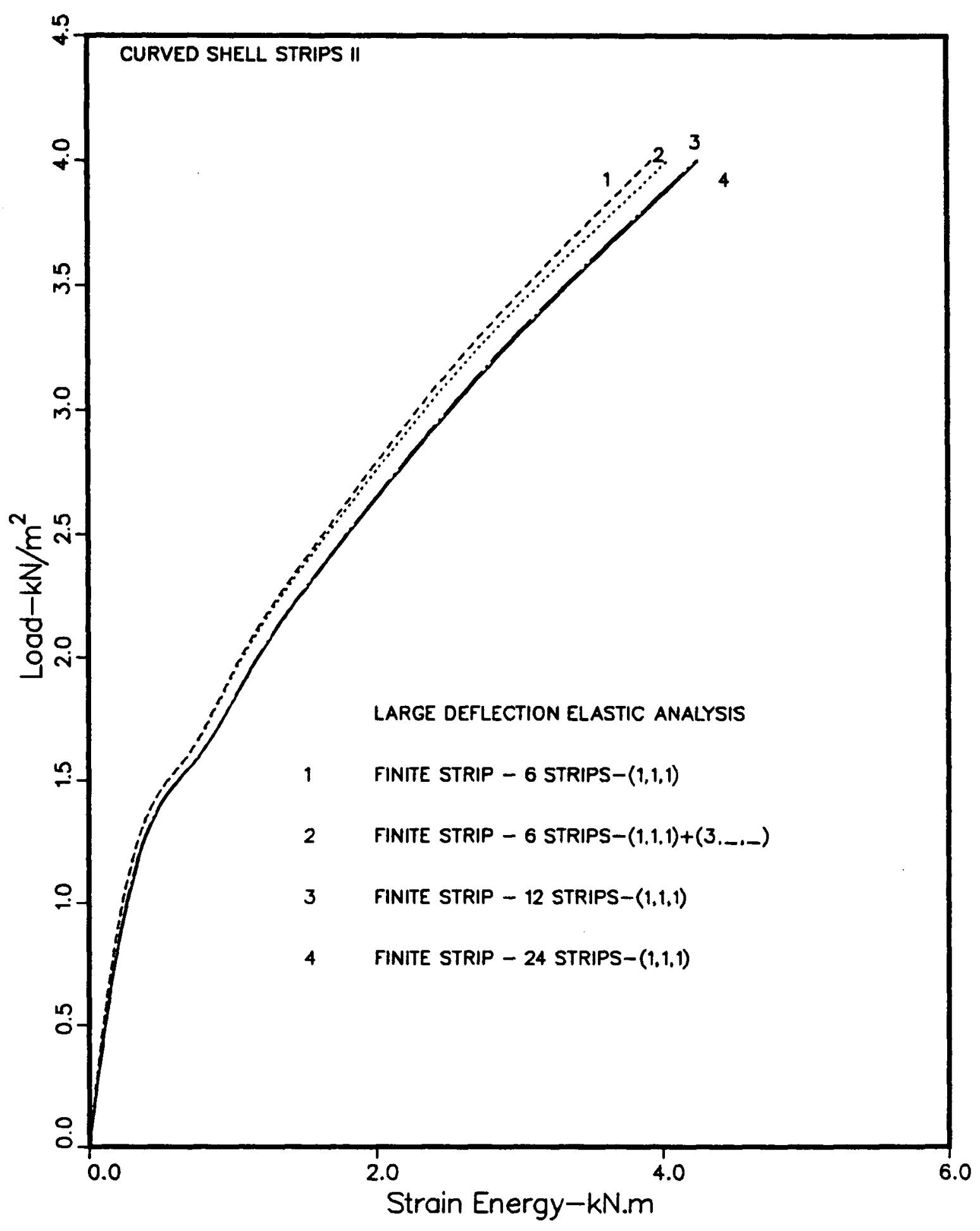

Figure 4.12: Variation of Strain Energy for the Shell-Roof problem 


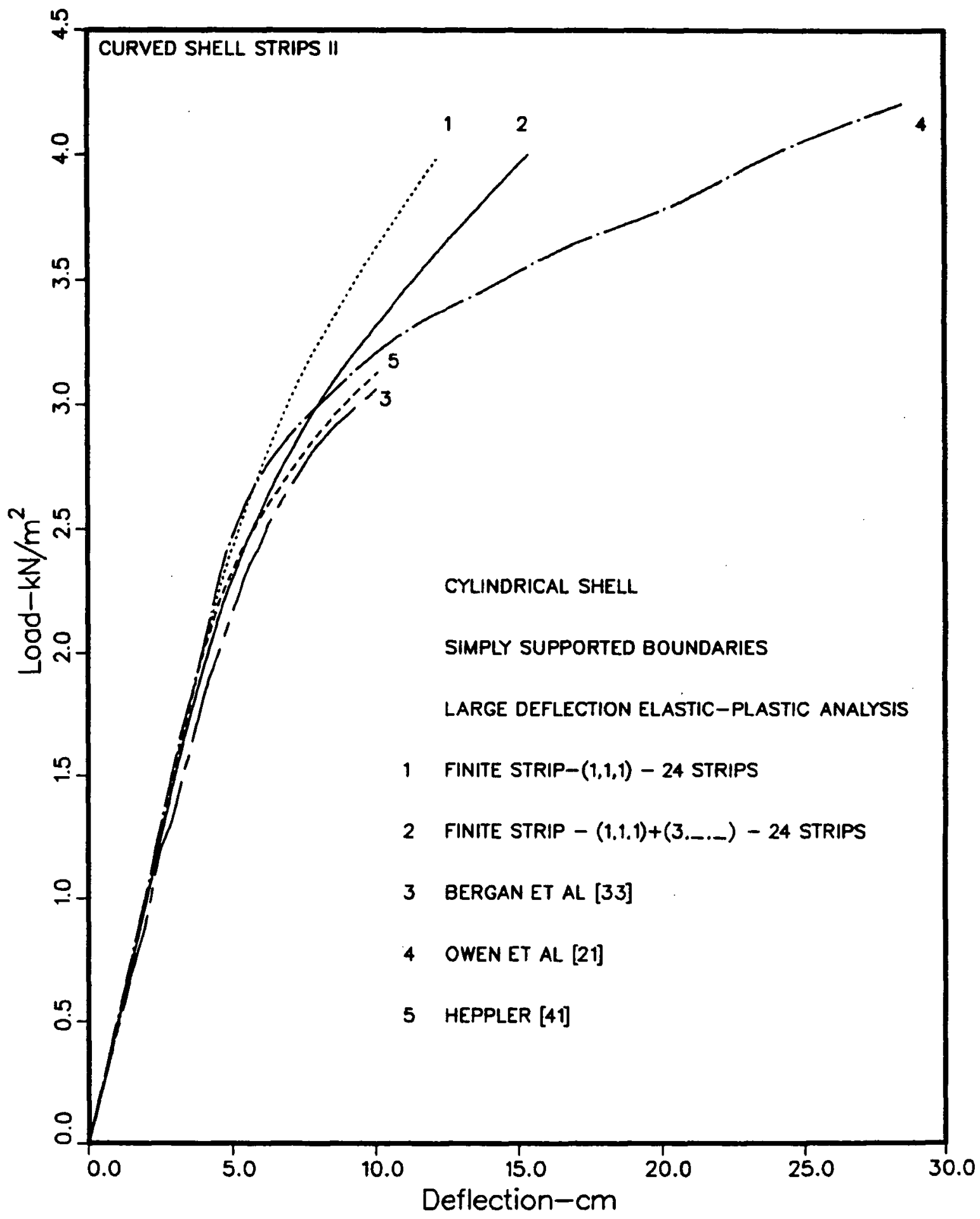

Figure 4.13: Vertical Displacement of the free edge 


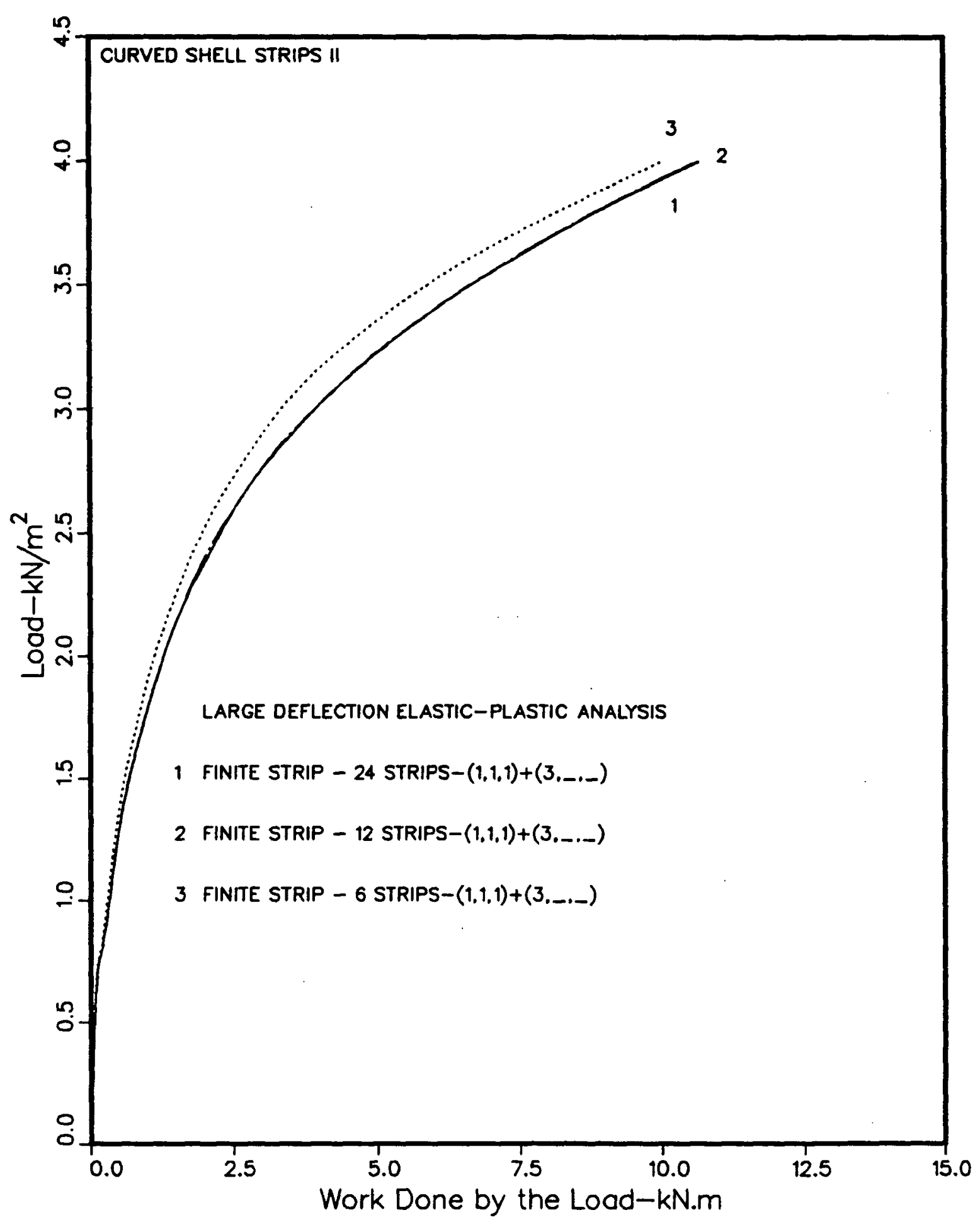

Figure 4.14: Variation of Work Done by the Load for the Shell-Roof problem 


\subsection{Clamped Cylindrical Shell - Analysis of}

\section{the Shell-Roof problem with Clamped}

\section{boundaries using Curved Shell strips II}

The cylindrical shell roof problem where the curved edges are clamped and the straight edges are free is now investigated. Fig. 4.15 depicts the problem configuration. The loading on the shell is its own weight. $X$

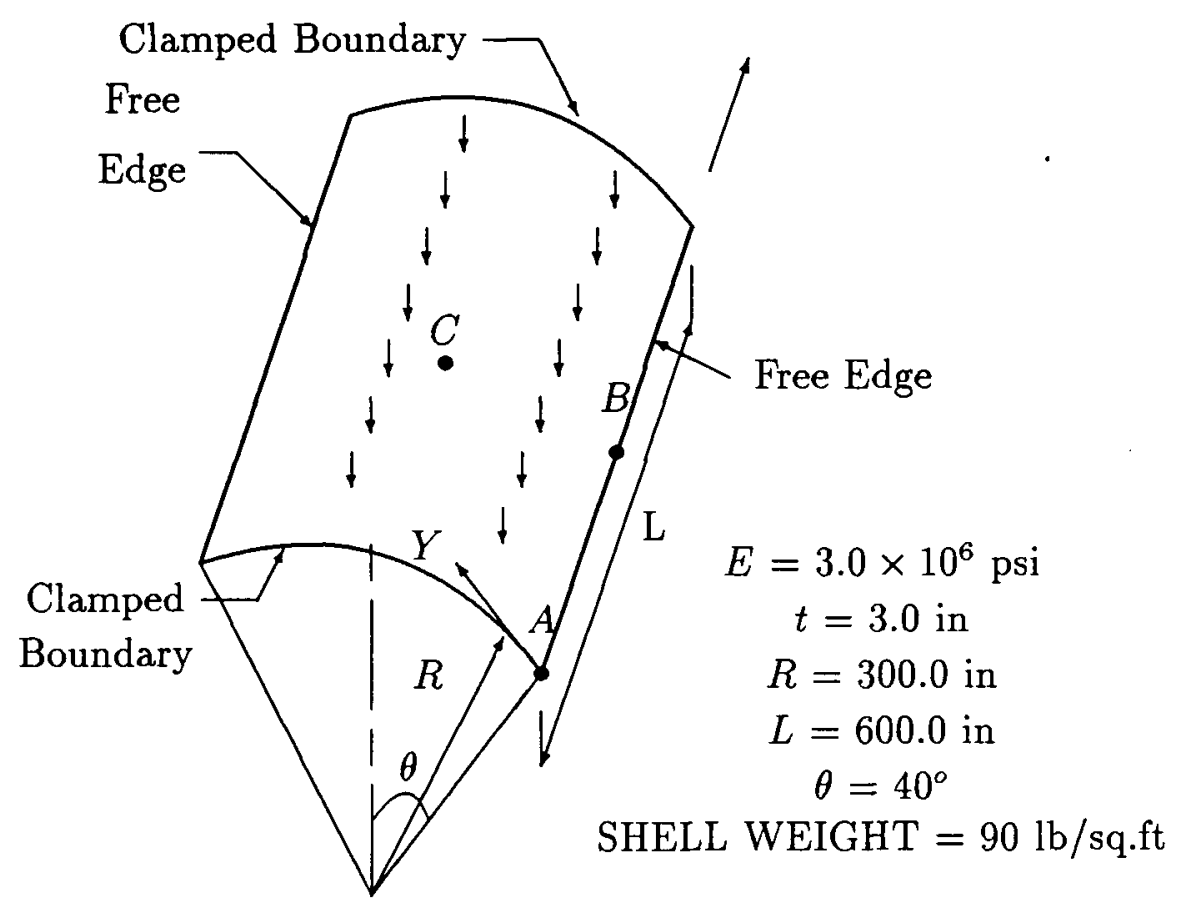

Figure 4.15: Clamped Shell problem

The longitudinal variation for the displacement components $u, v$ and $w$, for the clamped end conditions is given by: 


$$
\begin{array}{ll}
g_{m}^{u}(\xi)=\sin m \pi \xi ; & m=2,4,6, \cdots, \\
g_{n}^{v}(\xi)=\phi_{n}(\xi) ; & n=1,3,5, \cdots, \\
g_{k}^{v}(\xi)=\sin k \pi \xi ; & k=1,3,5, \cdots, \\
g_{p}^{w}(\xi)=\phi_{p}(\xi) ; & p=1,3,5, \cdots,
\end{array}
$$

where $\phi_{n}(\xi)$ represent the free vibration modes of a clamped-clamped beam.

These displacement functions satisfy the boundary conditions at the clamped ends of the shell which are:

$$
\begin{aligned}
u & =0 \\
v & =0 \\
w & =0 \\
\frac{\partial u}{\partial x} & =0
\end{aligned}
$$

The suitability of the $v$ mode is investigated by:

(a) allowing $v$ and $w$ to vary as $\phi(\xi)$.

(b) allowing the $v$ displacement to vary independently of $w$

$$
\begin{aligned}
& g_{1}^{v}(\xi)=\sin \pi \xi \\
& g_{1}^{w}(\xi)=\phi_{1}(\xi)
\end{aligned}
$$


It is evident that by choosing $\phi(\xi)$ as the longitudinal mode for $v$, in addition to satisfying zero displacement we would also satisfy $\frac{\partial v}{\partial x}=0$ at the clamped ends. Since $u$ is zero along the curved boundary, $\frac{\partial u}{\partial y}$ would also be identically zero and hence, the mid-surface shear strain would vanish at the clamped ends of the shell. By satisfying all displacement boundary conditions and a forced boundary condition at the clamped edges, we would overconstrain the problem.

The second choice of employing $\sin \pi \xi$ as a displacement mode for $v$ satisfies only the displacement boundary conditions and hence, is more acceptable. Obviously, by using both the displacement modes for $v$, we would be satisfying only the displacement boundary conditions. Test runs on the clamped cylindrical roof problem are carried out to determine the applicability of an appropriate mode.

Numerical investigations for the linear elastic analysis of the clamped shell were carried out by employing

(a) one mode for each of the displacement components $u, v$ and $w$ i.e.

$$
\begin{aligned}
& g_{1}^{u}(\xi)=\sin 2 \pi \xi \\
& g_{1}^{v}(\xi)=\phi_{1}(\xi) \\
& g_{1}^{w}(\xi)=\phi_{1}(\xi)
\end{aligned}
$$

In our notation, this analysis is referred to as $(2,1,1)$.

(b) two $u$ modes, one mode for $v$ and $w$ $g_{1}^{u}(\xi), g_{1}^{v}(\xi)$ and $g_{1}^{w}(\xi)$ are the same as in Eq. (4.14). The second $u$ mode is given by

$$
g_{2}^{u}(\xi)=\sin 4 \pi \xi
$$


This analysis, in our notation, would be referred to as $(2,1,1)+\left(4,_{-,-}\right)$.

(c) two $v$ modes, one mode for $u$ and $w$ $g_{1}^{u}(\xi), g_{1}^{v}(\xi)$ and $g_{1}^{w}(\xi)$ are the same as in Eq.( 4.14$)$. The second $v$ mode is given by

$$
g_{2}^{v}(\xi)=\sin \pi \xi
$$

This analysis, in our notation, would be referred to as $(2,1,1)+(-, 1,-)$.

(d) two modes for the in-plane displacements, $u$ and $v$, and one bending mode, $w$. $g_{1}^{u}(\xi), g_{1}^{v}(\xi)$ and $g_{1}^{w}(\xi)$ are the same as in Eq. (4.14). The second $u$ and $v$ modes are the same as in Eq. ( 4.15 ) and Eq. ( 4.16 ) respectively. This analysis, in our notation, would be referred to as $(2,1,1)+(4,1,-)$.

Table 4.8 presents the results of the linear elastic response of the clamped cylindrical shell where the finite strip analysis uses 12 strips for modelling the symmetric half of the shell. The finite strip solutions are compared with the results of the finite element analysis using a flat shell triangular element which couples the membrane action of the Allman element [34] with the bending action of Razzaque plate bending element [35]. A grid of 450 flat shell elements is used to model a quarter of the cylindrical panel. The results of the analysis for the vertical displacement of the free edge are plotted in Fig. 4.16.

In the finite element analysis, each flat shell element has 18 degrees of freedom. At the clamped end of the shell, all six nodal degrees of freedom are zeroed in the global co-ordinate system. This might contradict the boundary conditions at the clamped ends where it is expected that the rotation about the normal to the shell $\theta_{z}$ is non-zero in the element co-ordinate system. To simulate the boundary conditions at the clamped ends, the local $\theta_{z}$ degree of freedom should not be restrained. 


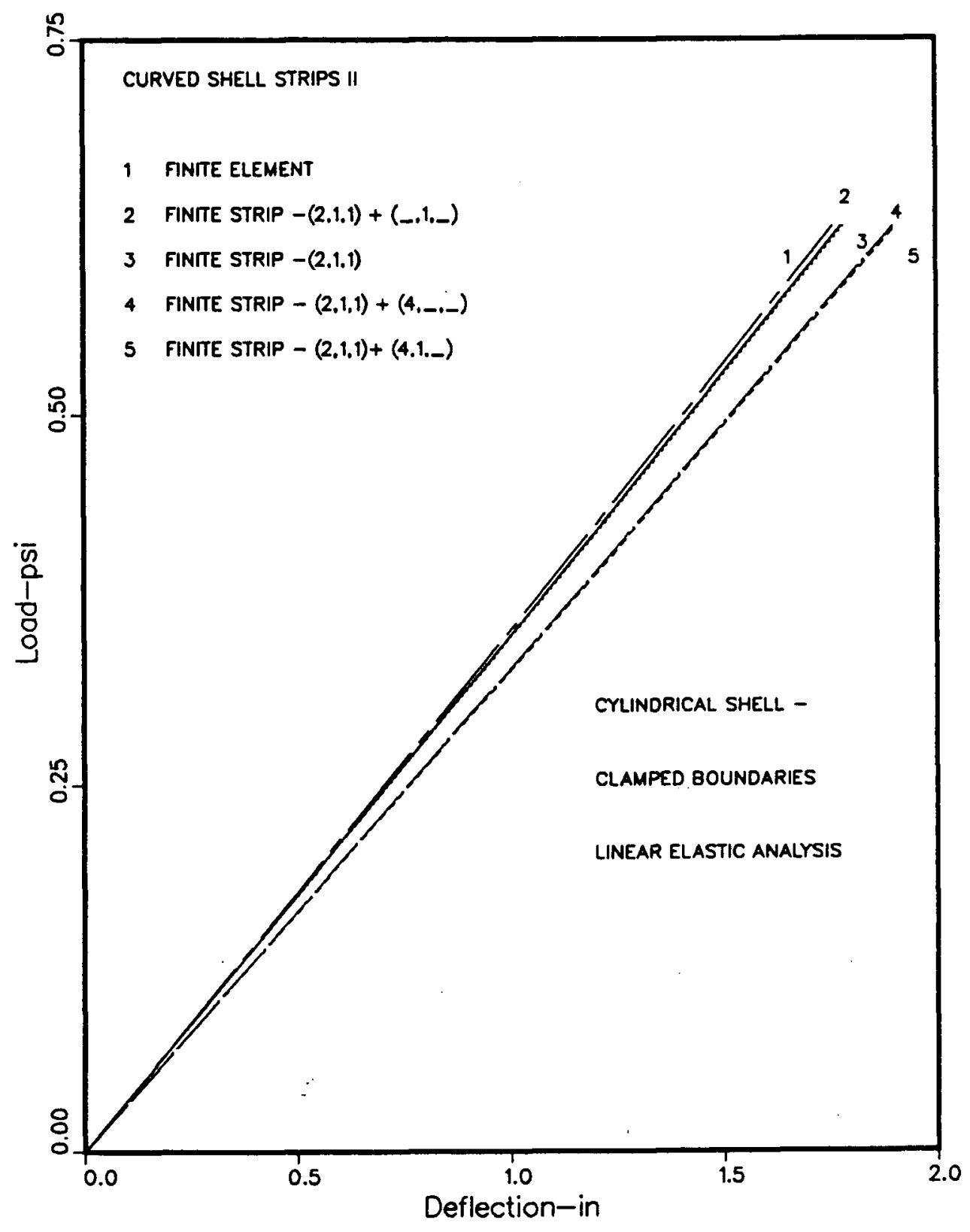

Figure 4.16: Vertical displacement of the free edge 
Table 4.8: Linear Elastic Response of the Clamped Shell Problem Using Curved Shell Strips II

\begin{tabular}{|c|c|c|c|c|}
\hline $\begin{array}{c}\text { Number of } \\
\text { modes }\end{array}$ & $\begin{array}{c}\mathrm{w}_{B} \\
\text { (in) }\end{array}$ & $\begin{array}{c}\mathrm{v}_{B} \\
\text { (in) }\end{array}$ & $\begin{array}{c}10 w_{C} \\
(\mathrm{in})\end{array}$ & $\begin{array}{c}\text { Strain Energy } \\
(\mathrm{K}-\mathrm{in})\end{array}$ \\
\hline$(2,1,1)$ & -1.785 & 1.0 & 2.77 & 19.25 \\
$(2,1,1)+(,, 1,-)$ & -1.78 & 1.0 & 2.74 & 19.56 \\
$(2,1,1)+\left(4,_{-,-}\right)$ & -1.907 & 1.06 & 3.02 & 20.74 \\
$(2,1,1)+(4,1,-)$ & -1.90 & 1.08 & 2.97 & 21.20 \\
Finite Element & -1.76 & 0.98 & 2.82 & 22.0 \\
\hline
\end{tabular}

In the present investigations, it is revealed that by not restraining the global $\theta_{Z}$, the results of the linear elastic analysis did not change significantly. The vertical displacement at the mid-span of the free edge remains unaffected while the change in strain-energy is within $1 \%$. These results indicate that the effect of zeroing the $\theta_{Z}$ degree of freedom is negligible and can therefore, be neglected.

The one mode finite strip solution compares very well with the results of the finite element analysis. The vertical displacement of the free edge is very slightly overpredicted, while the deflection at the top is underpredicted. This is consistent with other results that show a one mode approximation for $w$ overpredicts the central displacement. choice of the $w$ mode as in a clamped-clamped beam case.

By adding an extra $u$ or $v$ mode and subsequently two in-plane modes, the strain energy obtained from the finite strip analysis tends to approach the corresponding finite element solution. 
The justification of allowing the first $v$ mode to vary as $w$ in the longitudinal direction would be apparent if we examine the mid-surface strain-displacement relations of the cylindrical shell given by:

$$
\begin{aligned}
\epsilon_{x} & =\frac{\partial u}{\partial x} \\
\epsilon_{y} & =\frac{\partial v}{\partial y}+\frac{w}{R} \\
\gamma_{x y} & =\frac{\partial u}{\partial y}+\frac{\partial v}{\partial x}
\end{aligned}
$$

In the one-mode analysis, i.e. $(2,1,1)$, the avial strain would vary as $A_{1} \cos 2 \pi \xi$; the tangential strain would vary as $A_{2} \phi(\xi)$; and the shear strain would vary as $A_{3} \sin 2 \pi \xi\left(\right.$ since $\left.\frac{\partial \phi}{\partial x} \sim \sin 2 \pi \xi\right) ;$ where $A_{1}, A_{2}$ and $A_{3}$ are arbitrary constants.

Depending on the shape of the shell and the boundary conditions, the deformation of the shell may be nearly inextensional in some directions and extensional in other. For instance, if the shell is long and not supported along its straight edges, there is little membrane force in the tangential direction, and the load is carried through overall bending causing high membrane forces in the longitudinal direction. On the other hand, if the straight edges are supported, most of the load is carried through membrane forces in the tangential direction. For this reason it is necessary that the mid-surface or the membrane strains are able to represent both extensional and inextensional deformation.

Eq. ( 4.17 ) shows the mid-surface strain displacement relations. Since $\epsilon_{x}$ depends on $u$ there are no restrictions on the form of $u$. However, it is noted that $\epsilon_{y}$ depends on both $v$ and $w$, as does $\gamma_{x y}$ depend on $u$ and $v$. Thus, if $\epsilon_{y}$ is to be inextensional we must have the same longitudinal variation of $\frac{\partial v}{\partial y}$ and $w$. This then 
requires that $g_{n}^{v}(\xi)=g_{p}^{w}(\xi)$. Similarly if $\gamma_{x y}$ is to be inextensional (or zero), we must have $g_{m}^{u}(\xi)=\frac{\partial}{\partial \xi} g_{n}^{v}(\xi)$.

To allow for extensional modes, while at.the same time keeping the inextensional capacity in place, a second $v$ mode is introduced that has a different shape (in the longitudinal direction) than the first mode. Thus if $g_{1}^{v}(\xi)=\phi_{1}(\xi)$, then $g_{2}^{v}(\xi)=$ $\sin \pi \xi$.

For the shell just considered, there is probably not much membrane force in the tangential direction and so the introduction of a second $v$ mode does not have much effect. Since most of the load is carried by membrane forces in the longitudinal direction, the introduction of a second $u$ mode makes a much larger change to the deflections. (see Table 4.8).

A plot of the variation of the displacement patterns of $v$ and $w$ in the longitudinal direction is shown in Fig. 4.17 along with the results from the finite element analysis.

\subsection{Non-Linear Analysis of the Clamped-Shell}

\section{problem}

\subsubsection{Linear Elastic-Plastic Analysis}

The effect of material non-linearities on the response of the clamped cylindrical panel was investigated. The shell material was assumed to be elastic-perfectly plastic. The analysis was carried out using $2 v$ modes and a single mode for $u$ and $w,(2,1,1)+\left({ }_{-,} 1,-\right)$ in our notation. 

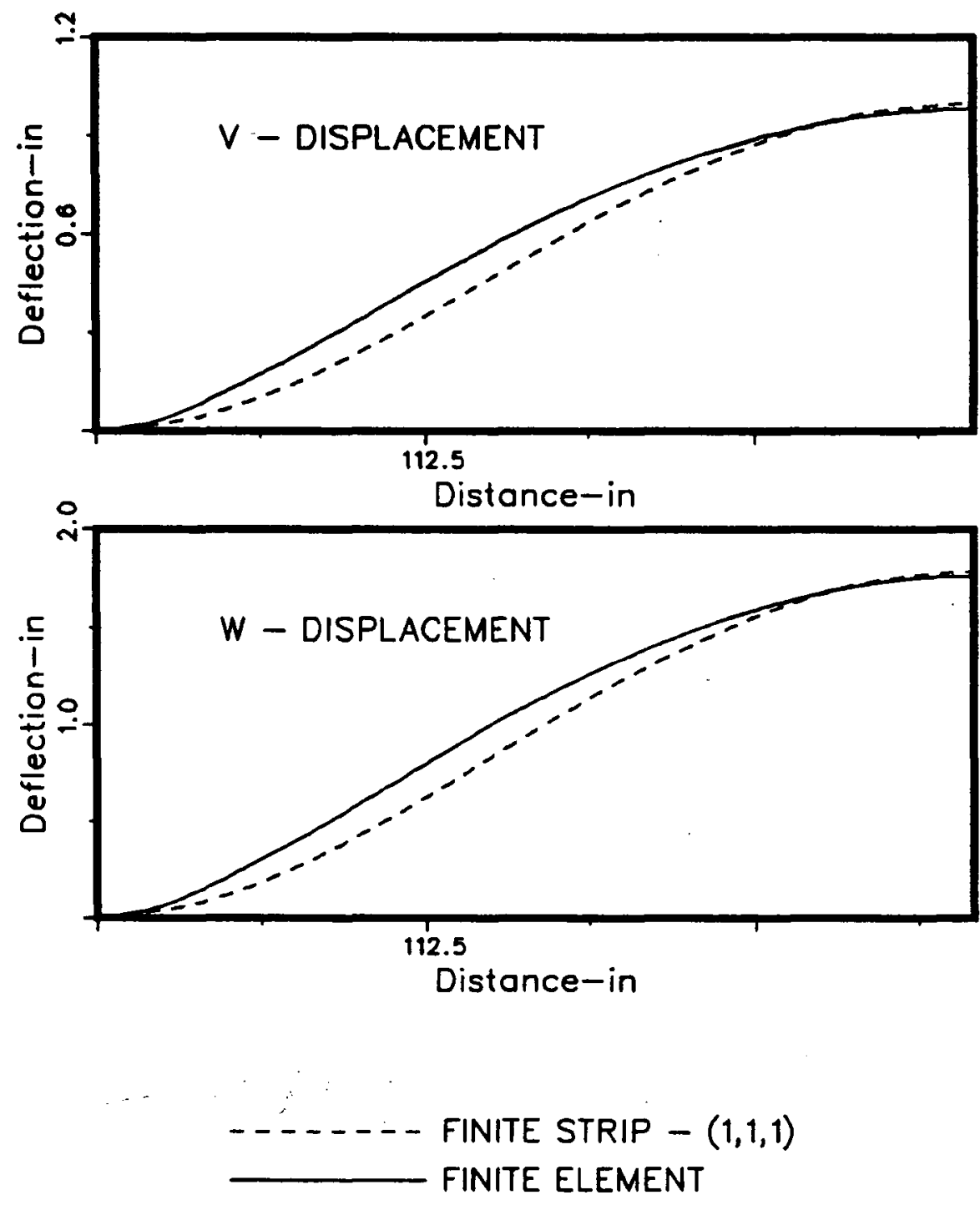

Figure 4.17: Variation of $v$ and $w$ displacements for the Clamped-Shell Problem 
Fig. 4.19 depicts the plot of load level, $p$, versus the displacement, $w_{B}$. Initial yield takes place at the clamped edge at a load of 0.2 psi. This is consistent with the formation of the first plastic hinge at the clamped end of a fixed-fixed beam. For a maximum load level of $0.625 \mathrm{psi}$, the response curve has a finite slope. A comparison with the response of the shell-roof problem in Fig. 4.9 indicates that considerable yielding of the shell material had occurred in that shell i.e. when the curved edges were diaphram supported. However, the response curve in the case of clamped boundaries shows that the shell material is still largely elastic.

\subsubsection{Large Deflection Elastic Analysis}

Using the same modes and strips as above the load-deflection response of the cylindrical panel is depicted in Fig. 4.18. From the curve, it can be seen that the vertical displacement of the free edge is $3 \%$ stiffer than the corresponding displacement for the linear elastic case at a load level of 0.625psi. Apparently, the effect of large deformations is not very pronounced at such a low load level because the maximum displacement is only about one-half of the thickness. By increasing the maximum Joad level to $1.6 p s i$, the effect of large deformations is obvious. A relative stiffening of strain energy of about $10 \%$ shows a similar trend as the diaphram supported shell-roof problem.

\subsubsection{Large Deflection Elastic-Plastic Analysis}

The effect of incorporating both,material and geometric non-linearities, in the analysis of the clamped cylindrical shell is now investigated. The finite strip analysis could be represented as $(2,1,1)+(-, 1,-)$ in our notation. Fig. 4.19 depicts a plot of 


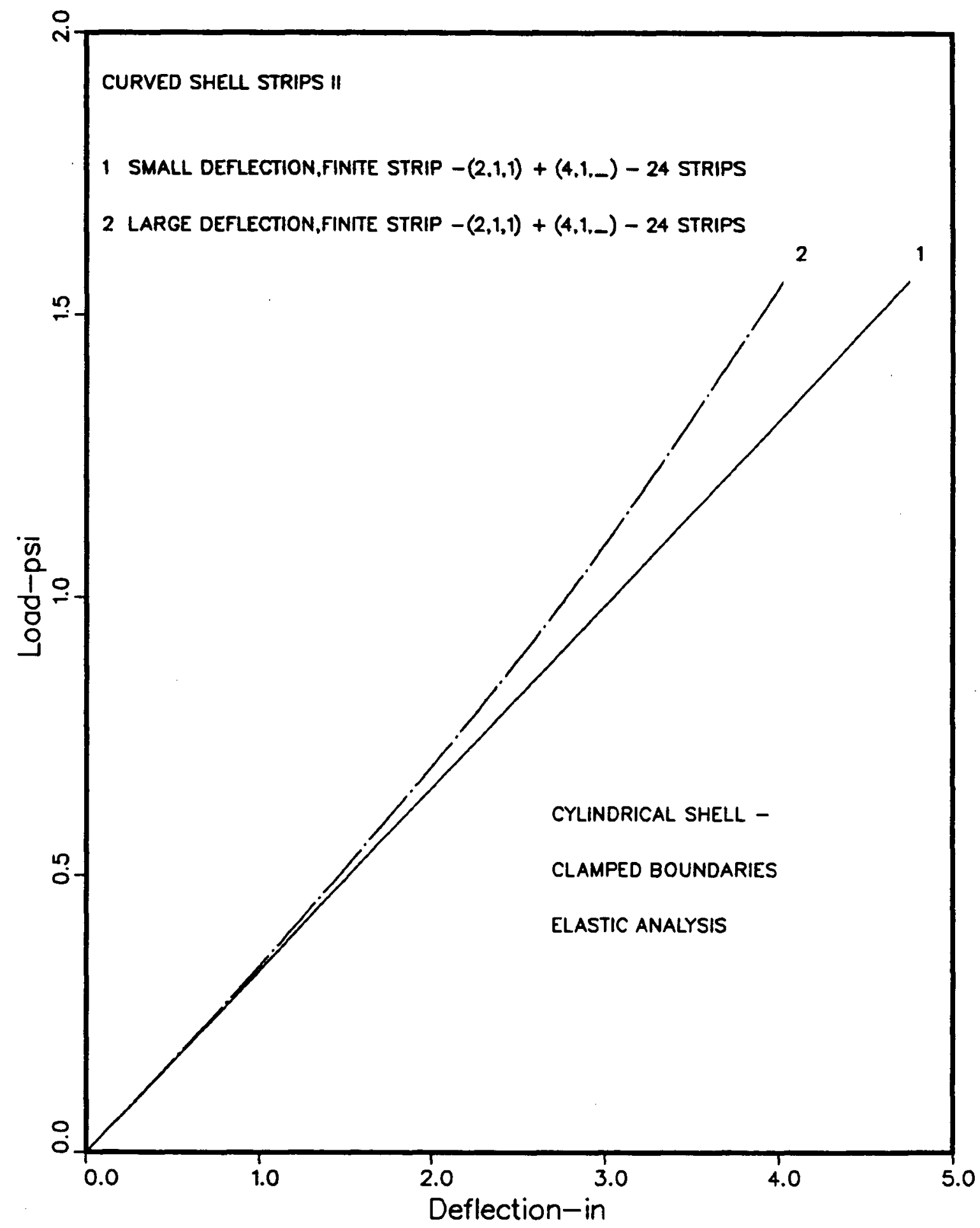

Figure 4.18: Vertical Displacement of the free edge 


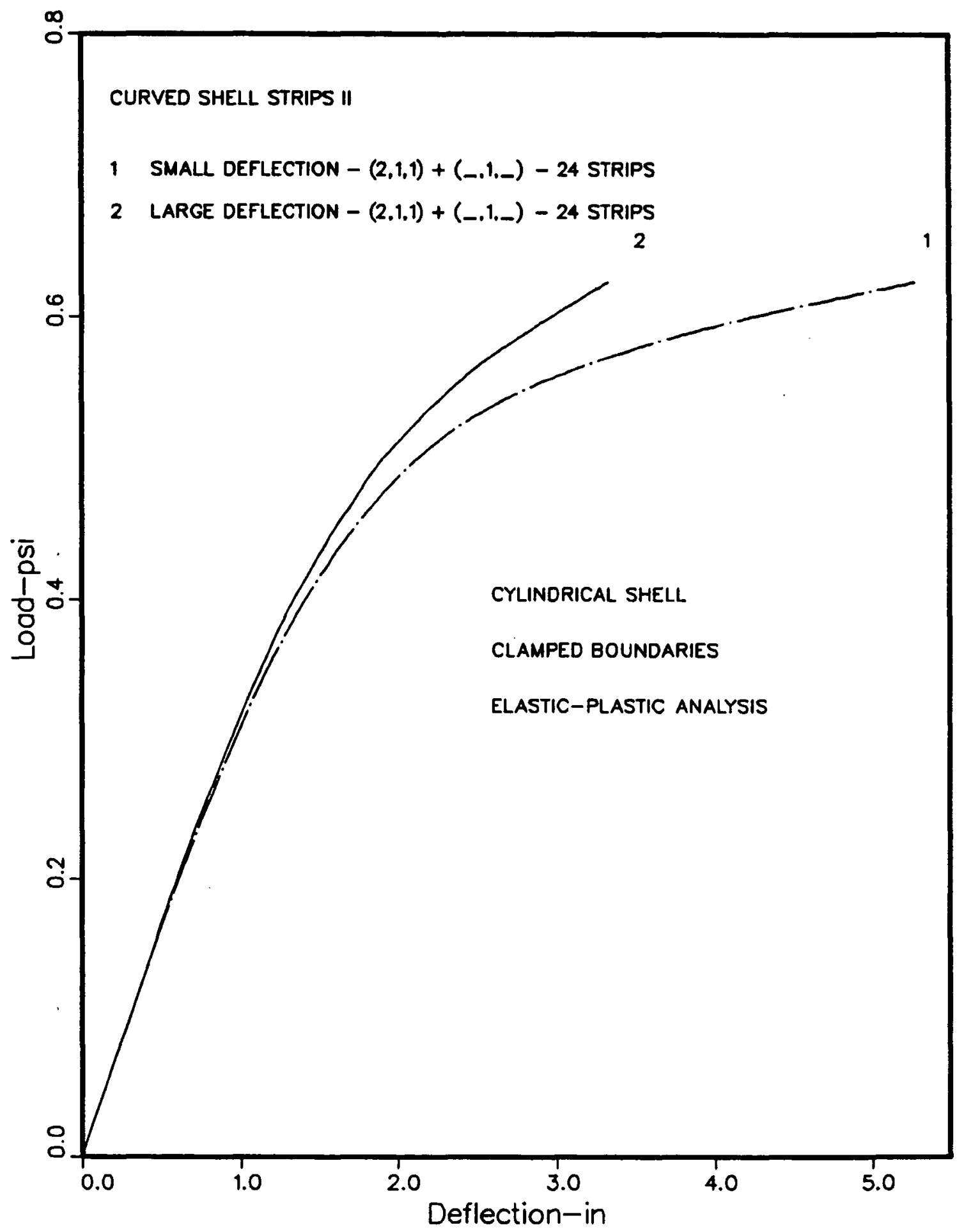

Figure 4.19: Vertical Displacement of the free edge 
the vertical displacement of the free edge versus the load. Initial yield takes place at a load of $0.23 p s i$ at the clamped boundary. Due to the non-availability of any comparisons the response curve is compared with the corresponding response of the diaphram supported shell. An examination of the two curves shows a similar trend.

\subsection{Analysis of a Cylindrical Shell}

\section{Clamped along all Boundaries}

In the last example problem, the boundary conditions along the straight edges of the shell were free. The curved edges were clamped. The effect of clamping the straight edges of the shell is now investigated. Fig. 4.20 shows the configuration of the problem. This shell is very flat, square in developed plan form, and very thin with an $R / t$ ratio of 800 . A uniform pressure load is applied on the cylindrical shell radially inwards.

Finite element analyses of the cylindrical shell were carried out by Hughes et al [37] using a degeneration concept of reducing a 3-D continuum element by satisfying the shell approximations and by Owen et al [20]. The linear and large deflection elastic analyses were carried out up to a load of $1379 \mathrm{~N} / \mathrm{m}^{2}(0.2 \mathrm{psi})$. Noor [36] analyzed the same problem using a reduction method - which in essence, is a technique to limit the deformation modes of the discretized structure to some known modes. The analyses was carried out up to a load level of $2715 \mathrm{~N} / \mathrm{m}^{2}$ (cor-

responding to a load parameter, $\frac{p R}{E t}$, of $7.0 \times 10^{-4}$ ) both for the linear and the large deflection elastic case. In the finite element analysis Noor [36] used 7 basis vectors or displacement modes. 


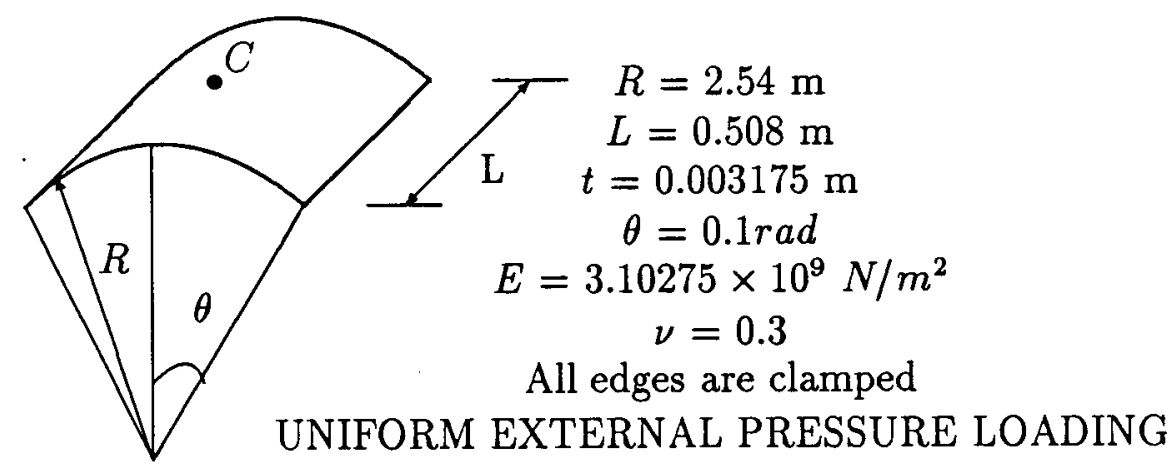

Figure 4.20: Clamped-Clamped Shell

The finite strip analysis, using a 12 strip discretization for the symmetric half of the shell, has been carried out up to a load level of $2715 \mathrm{~N} / \mathrm{m}^{2}\left(\frac{p R}{E t}=7.0 \times 10^{-4}\right)$. As before, the displacement modes in the longitudinal direction could be represented as $(2,1,1)+(-, 1,-)$ in our notation. The results of the linear elastic analysis were found to be in very good agreement with the finite element solutions $[36],[37],[20]$. In the finite strip analysis, it was seen that for the two $v$ modes, the sine mode dominated the $\phi$ mode by about a factor of 5 indicating that extension in the tangential direction was important. The response of the cylindrical panel is plotted in Fig. 4.21 for the linear elastic as well as the large defiection elastic case. A non-dimensional displacement parameter $\frac{w_{c}}{R}$ is plotted against a non-dimensional load parameter, $\frac{p R}{E t}$ where, $w_{c}$ is the vertical displacement of the crown and $p$ is the load level. It is apparent from the plot that the non-linear response of the shell 


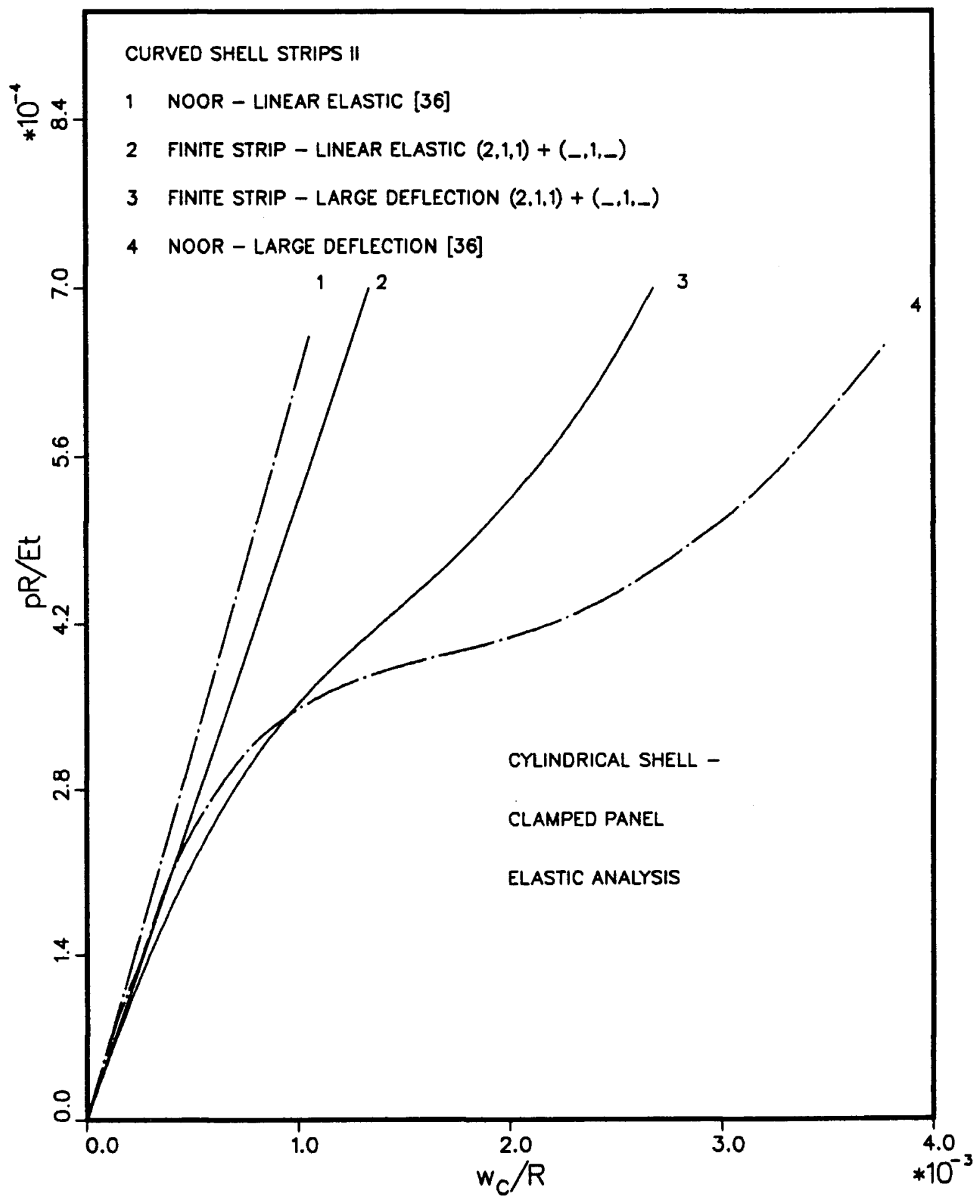

Figure 4.21: Vertical Displacement of the crown 
is on the flexible side of the corresponding linear response at nearly all load levels for both the finite strip and Noor's analysis. Since the extensional mode dominates in the tangential direction, it seems that at lower load levels most of the load is carried by membrane forces in this direction. Also, membrane softening seems to occur until at high load levels, membrane stiffening in the longitudinal direction prevents eventual collapse of the shell.

The finite strip solution follows the same trend as the finite element solutions. However, the finite strip solution is slightly stiffer than the corresponding finite element solution [36]. Obviously the finite element analysis leads to a more comprehensive solution and is more accurate than the finite strip solution which is essentially only a one mode (for bending) solution. 


\section{Chapter 5}

\section{Summary, Conclusions and}

\section{Suggestions for Future Research}

\subsection{Summary and Conclusions}

A scientific study of living things provides innumerable examples where nature has evolved efficient structural forms with maximum economy relative to the forces resisted by the structure. The availability of new structural materials has brought about a revolution in structural design and the practicability of these materials is currently being investigated. However, the strength of a structure is not only determined by the material used, but is also dependent on the structural form. It has been concluded by researchers that structural materials are generally far more efficient in an extensional mode rather than a flexural mode [39]. Shell structures fall into this category of structures. The efficient load resisting mechanism in shell structures results from developing an extensional mode through an initial curvature.

The analytical methods of analysis of cylindrical shells are too cumbersome and it is impractical to obtain a complete non-linear solution. Therefore, numerical procedures have to be adopted for the prediction of the complete non-linear response of cylindrical shells. In the preceding chapters, a numerical procedure based on the 
finite strip method, has been developed for the non-linear analysis of cylindrical shells.

In the finite strip method, the cylindrical shell is discretized into several finite strips with the strip direction parallel to the shell axis. The variation of the displacement components along the strip direction is determined by employing one or two modes of continuously differentiable functions. These modes satisfy the boundary conditions at the ends of the strip. Polynomial functions represents the variation of displacements in the transverse direction. Numerical integration of the equilibrium equations necessary for obtaining the stiffness matrices and the load vector is carried out by adopting Gaussian quadrature. Equilibrium equations for a single finite strip are obtained by using the virtual work principle. The global equilibrium equations are subsequently obtained by assembling the equilibrium equations for each finite strip. The resulting set of equations is solved by Gauss elimination. For the non-linear analyses, the resulting set of non-linear equations is solved via the Newton-Raphson iterative scheme, starting with the linear solution.

Various example problems were tested to verify the practicability of the finite strip modelling of the cylindrical shell panels. In the first stage of the investigation, the linear elastic response of the axisymmetric cylindrical shell problem was compared with the analytical solution. From a parametric study conducted to determine the accuracy of the finite strip solution, it was found that the finite strip solution obtained by employing one bending mode yields reasonable accuracy for shells with an effective boundary layer width exceeding $L / 5$. For a smaller value of the effective boundary layer width, it was felt that additional bending modes were necessary to approximate the analytical solution. The classical shell-roof problem was analyzed by employing a single mode for the displacement components and 
a very good estimate of deflections and strain-energy was obtained. For a small deflection analysis, both elastic and elastic-plastic, good agreement of results was observed between the finite strip and the finite element and analytical solutions. However, when the effect of large deflections was incorporated, it was found necessary to employ an additional $u$ mode.

The effect of clamping the boundaries of the shell was also investigated. The finite strip solutions were obtained by employing two $v$ modes and a single $u$ and $w$ mode. The additional $v$ mode was necessary to represent the inextensional deformations of the shell. The inextensible $v$ mode was found to dominate when the straight edges of the shell were free, i.e. the flexural action serves as a primary load carrying mechanism. The second $v$ mode dominated when the straight edges of the shell were clamped, i.e the arch action serves as a primary load carrying mechanism. 'The finite strip solutions for both types of boundary conditions along the straight edges - free or clamped, were in very good agreement with the respective finite element solutions for the linear elastic analysis.

A complete large deflection, elastic-plastic analysis of the cylindrical shell-roof problem with clamped curved boundaries and free longitudinal boundaries was also carried out. The resulting variation of displacement components was relatively stiffer as compared with the response of the diaphram supported shell. For elastic-plastic analysis, it was apparent that the first yield occurs at the clamped boundaries.

The finite strip solution for the case of a cylindrical shell clamped along all edges was compared with the finite element solution for a large deflection elastic analysis. The displacement results were found to be in reasonably good agreement. 


\subsection{Suggestions for Future Research}

A numerical procedure based on the finite strip method has been presented for the static analysis of cylindrical shells in the preceding chapters. It would be worthwhile to determine the response by employing additional bending modes, especially when the boundaries of the cylindrical shell are clamped. The finite strip analysis should also be extended to determine the dynamic response of cylindrical shells.

The analysis could easily be extended to analyze longitudinally stiffened shells which are being used extensively in ship structures, aircraft and submarines. To keep pace with the modern trend of using lighter composite materials from considerations of economy, the finite strip analysis should be extended to these materials as well. 


\section{Appendix A}

\section{Load Vector for Curved Shell II}

\section{Formulation}

In Chapter 3, it was stated that the consistent load vector for a self-weight analysis for the Curved Shell strip II is obtained by integrating the load with the shape functions in both, the longitudinal and transverse direction, in closed form.

The uniformly distributed vertical load will be resolved into tangential and radial directions and then integrated with the respective shape functions in the two directions. Therefore, the element load vector for the $j$ th strip in the tangential direction will be written as:

$$
\left(p_{i}^{v}\right)_{j}=\int_{A} N_{i}^{v} p \sin \alpha_{j} \mathrm{~d} \mathbf{A}
$$

where $i=1,2,3$.

The element load vector for the $j$ th strip in the radial direction is given by:

$$
\left(p_{i}^{w}\right)_{j}=\int_{A} N_{i}^{w} p \cos \alpha_{j} \mathrm{dA}
$$

where $i=1,2,3,4$.

The shape functions $N_{i}^{v}$ and $N_{i}^{w}$ are given in Eq.( 3.42$)$. After integration in closed form, the terms in the element load vector for the $j$ th strip in the tangential 
direction are written as:

$$
\begin{aligned}
\left(p_{1}^{v}\right)_{e}= & \frac{2}{\pi} R L p\left(\cos (n-i) \alpha_{j}\left\{\frac{-4}{\alpha_{j}^{2}}\right\}-\cos (n-i+1) \alpha_{j}\left\{1-\frac{4}{\alpha_{j}^{2}}\right\}\right. \\
& \left.+\sin (n-i) \alpha_{j}\left\{\frac{1}{\alpha_{j}}\right\}-\sin (n-i+1) \alpha_{j}\left\{\frac{-3}{\alpha_{j}}\right\}\right) \\
\left(p_{2}^{v}\right)_{e}= & \frac{2}{\pi} R L p\left(\cos (n-i) \alpha_{j}\left\{\frac{8}{\alpha_{j}^{2}}\right\}-\cos (n-i+1) \alpha_{j}\left\{\frac{8}{\alpha_{j}^{2}}\right\}\right. \\
& \left.+\sin (n-i) \alpha_{j}\left\{\frac{-4}{\alpha_{j}}\right\}-\sin (n-i+1) \alpha_{j}\left\{\frac{4}{\alpha_{j}}\right\}\right) \\
\left(p_{3}^{v}\right)_{e}= & \frac{2}{\pi} R L p\left(\cos (n-i) \alpha_{j}\left\{1-\frac{4}{\alpha_{j}^{2}}\right\}-\cos (n-i+1) \alpha_{j}\left\{\frac{-4}{\alpha_{j}^{2}}\right\}\right. \\
& \left.+\sin (n-i) \alpha_{j}\left\{\frac{3}{\alpha_{j}}\right\}-\sin (n-i+1) \alpha_{j}\left\{\frac{-1}{\alpha_{j}}\right\}\right)
\end{aligned}
$$

The element load vector in the radial direction for the $j$ th strip is written as:

$$
\begin{aligned}
\left(p_{1}^{w}\right)_{e}= & \frac{2}{\pi} R L p\left(\sin (n-i) \alpha_{j}\left\{\frac{6}{\alpha_{j}^{2}}\right\}-\sin (n-i+1) \alpha_{j}\left\{-1-\frac{6}{\alpha_{j}^{2}}\right\}\right. \\
& \left.+\cos (n-i) \alpha_{j}\left\{\frac{-12}{\alpha_{j}^{3}}\right\}-\cos (n-i+1) \alpha_{j}\left\{\frac{-12}{\alpha_{j}^{3}}\right\}\right) \\
\left(p_{2}^{w}\right)_{e}= & \frac{2}{\pi} R L b p\left(\sin (n-i) \alpha_{j}\left\{\frac{2}{\alpha_{j}^{2}}\right\}-\sin (n-i+1) \alpha_{j}\left\{\frac{-4}{\alpha_{j}^{2}}\right\}\right. \\
& \left.+\cos (n-i) \alpha_{j}\left\{\frac{-6}{\alpha_{j}^{3}}\right\}-\cos (n-i+1) \alpha_{j}\left\{\frac{1}{\alpha_{j}}-\frac{6}{\alpha_{j}^{3}}\right\}\right) \\
\left(p_{3}^{w}\right)_{e}= & \frac{2}{\pi} R L p\left(\sin (n-i) \alpha_{j}\left\{-1-\frac{6}{\alpha_{j}^{2}}\right\}-\sin (n-i+1) \alpha_{j}\left\{\frac{6}{\alpha_{j}^{2}}\right\}\right. \\
& \left.+\cos (n-i) \alpha_{j}\left\{\frac{12}{\alpha_{j}^{3}}\right\}-\cos (n-i+1) \alpha_{j}\left\{\frac{12}{\alpha_{j}^{3}}\right\}\right) \\
\left(p_{4}^{w}\right)_{e}= & \frac{2}{\pi} R L b p\left(\sin (n-i) \alpha_{j}\left\{\frac{4}{\alpha_{j}^{2}}\right\}-\sin (n-i+1) \alpha_{j}\left\{\frac{-2}{\alpha_{j}^{2}}\right\}\right. \\
& \left.+\cos (n-i) \alpha_{j}\left\{\frac{1}{\alpha_{j}}-\frac{6}{\alpha_{j}^{3}}\right\}-\cos (n-i+1) \alpha_{j}\left\{\frac{-6}{\alpha_{j}^{3}}\right\}\right)
\end{aligned}
$$


where $n$ is the total number of finite strips used in modelling the symmetrical half of the shell, $\alpha_{j}$ is the angle subtended by $j$ th finite strip at the center of curvature measured with respect to the vertical, $R$ is the radius of curvature of the cylindrical shell and $L$ and $b$ are the length and the width of the strip respectively. 


\section{Appendix B}

\section{Derivation of the $Q$ matrix}

In Chapter 3, the $Q$ matrix was defined as:

$$
[Q]=\left(\frac{\partial[C]^{T}}{\partial\{\Delta\}}\right)\{\sigma\}
$$

Let us derive the $[Q]$ matrix for a one-mode solution for the Curved Shell strips II. Then the $[C]$ matrix would be a $3 \times 10$ matrix while $\{\Delta\}$ would be a column vector of nodal displacements of size $10 \times 1$. Then, $\left(\frac{\partial[C]^{T}}{\partial\{\Delta\}}\right)$ would result in a three-dimensional array $A$ of size $10 \times 10 \times 3$. The elements of $A$ are given by,

$$
A_{i k j}=\frac{\partial\left[C_{i k}\right]^{T}}{\partial\left\{\Delta_{j}\right\}}
$$

with $i, j=1,2 \cdots, 10$ and $k=1,2,3$. Hence, the $[Q]$ matrix could be written as:

$$
[Q]=[A]\{\sigma\}
$$

where,

$$
Q_{i j}=\sum_{k=1}^{3} \frac{\partial\left(C^{T}\right)_{i k}}{\partial\{\Delta\}_{j}} \sigma_{k}
$$


The terms in the $Q$ matrix are as follows:

$$
[Q]=\left[\begin{array}{cccccccccc}
0 & 0 & 0 & 0 & 0 & 0 & 0 & 0 & 0 & 0 \\
0 & 0 & 0 & 0 & 0 & 0 & 0 & 0 & 0 & 0 \\
0 & 0 & A_{11} & A_{12} & 0 & 0 & 0 & 0 & A_{13} & A_{14} \\
0 & 0 & A_{21} & A_{22} & 0 & 0 & 0 & 0 & A_{23} & A_{24} \\
0 & 0 & 0 & 0 & 0 & 0 & 0 & 0 & 0 & 0 \\
0 & 0 & 0 & 0 & 0 & 0 & 0 & 0 & 0 & 0 \\
0 & 0 & 0 & 0 & 0 & 0 & 0 & 0 & 0 & 0 \\
0 & 0 & 0 & 0 & 0 & 0 & 0 & 0 & 0 & 0 \\
0 & 0 & A_{31} & A_{32} & 0 & 0 & 0 & 0 & A_{33} & A_{34} \\
0 & 0 & A_{41} & A_{42} & 0 & 0 & 0 & 0 & A_{43} & A_{44}
\end{array}\right]
$$

where,

$$
\begin{aligned}
& A_{i j}=N_{i, x}^{w} N_{j, x}^{w} \sigma_{x}+N_{i, y}^{w} N_{j, y}^{w} \sigma_{y}+\left(N_{i, x}^{w} N_{j, y}^{w}+N_{i, y}^{w} N_{j, x}^{w}\right) \tau_{x y}, \quad i, j .=1,2,3,4 \\
& \text { and }
\end{aligned}
$$

$$
\begin{aligned}
& N_{i, x}^{w}=\frac{\partial N_{i}^{w}}{\partial x}, \\
& N_{i, y}^{w}=\frac{\partial N_{i}^{w}}{\partial y}, \\
& N_{j, x}^{w}=\frac{\partial N_{j}^{w}}{\partial x} \\
& N_{j, y}^{w}=\frac{\partial N_{j}^{w \prime}}{\partial y} .
\end{aligned}
$$




\section{Bibliography}

[1] Vlasov, V.Z., General Theory of Shells and their Applications in Engineering, NASA TTF-99, 1949.

[2] Love, A.E.H., A Treatise on the Mathematical Theory of Elasticity, Fourth Edition, Dover Publications, Inc., New York, 1944.

[3] Flügge, W., Stresses in Shells, Second Edition, Springer-Verlag, New York, 1973.

[4] Timosheriko, S.P., and S.W.Kreiger, Theory of Plates and Shells, Second Edition, McGraw-Hill Book Company, 1983.

[5] Donell, L.H., and C.C.Wan, Effect of Imperfections on Buckling of thin Cylinders under External Pressure, J. Appl. Mech., 23, No.4, pp 569, 1956.

[6] Tene.,Y. and M. Epstein, A Direct Variational Formulation of Non-Linear Shell Theory, Proceedings of the International Conference on Variational Methods in Engng., Southampton, pp 7/46-7/65, 1972.

[7] Wempner, G., Discrete Approximations of Elastic-Plastic bodies by Variational Methods, Proceedings of the International Conference on Variational Methods in Engng., Southampton, pp 7/1-7/35, 1972.

[8] Hellinger, E., Die Alegmeinen Ansatze der Kontinua, Encyklopädie der Mathematischen Wissenschaften, 4, No.4, 1914.

[9] Hu, H.C., On some Variational Principles in the Theory of Elasticity and Plasticity, Scientia Sinica, 4, 1955. 
[10] Washizu, K., On some Variational Principles in the Theory of Elasticity and Plasticity, Tech. Report 25-18, Mass. Inst. Tech., 1955.

[11] Reissner, E., On a Variational theorem in Elasticity, J. Math. Phy., 29,1950.

[12] Cook, R.D., Concepts and Applications of Finite Element Analysis, Second Edition, John Wiley and sons, New York, 1981.

[13] Crisfield, M.A., Linear and Non-Linear Analysis of Cylindrical Shells, TRRL Laboratory Report 987, Transport and Road Research Laboratory, Crowthorne, Berkshire, U.K., 1981.

[14] Koiter, W.T., A consistent first-approximation in the general theory of thin elastic shells, First IUTAM Symposium, North Holland Company, Amesterdam, 12, 1960.

[15] Budiansky, B. and J.L.Sanders, On the 'best' first-order linear theory of thin elastic shells, Progress in Applied Mechanics, The Prager Anniversary Volume, pp $127,1963$.

[16] Irons, B.M., The semi-loof element, Finite Elements for thin shells and Curved members, Ch.11, Wiley, New York, 1976.

[17] Ergatoudis, J.G., Isoparametric finite elements in two- and three-dimensional analysis, Ph.D. thesis, University of Wales, Swansea, 1968.

[18] Ahmad, S., Curved finite elements in the analysis of solid shells and plate structures, Ph.D. thesis, University of Wales, Swansea, 1969.

[19] Zienkiewicz, O.C., R. L. Taylor and J.M.Too, Reduced Integration technique in the general analysis of plates and shells, Int. J. Num. Meth. Engng.,13, pp 275-290, 1974.

[20] Martin, R.A.F. and D.R.J. Owen, Elastoplastic and Geometrically non-linear 
thin shell analysis by the semiloof element, Comp. and Struct., 13, pp 505-513, 1981.

[21] Figueriras, J.A., and D.R.J.Owen, Analysis of Elasto-Plastic and Geometrically Non-Linear Anisotropic Plates and Shells, Finite Element Software for Plates and Shells, 1984.

[22] Cheung, Y.K., Finite Strip Method in Structural Analysis, Permagon Press, 1976.

[23] Cheung, Y.K., The Finite Strip Method in the Analysis of Elastic Plates with two Opposite Simply Supported Ends, Proceedings, The Institution of Civil Engineers, London, 40, pp 1-7, 1968.

[24] Abayakoon, S.B.S., Large Deflection Elastic-Plastic Analysis of Plate Structures by the Finite Strip Method, Ph.D. thesis, University of British Columbia, Vancouver, 1987.

[25] Dawe, D.R.J., Static Analysis of Diaphgram Supported Cylindrical Shells using a Curved Finite Strip, Int. J. Num. Meth. Engng. 11, pp 1347-1364, 1977.

[26] Cheung, Y.K., S.C.Fan and C.Q. Wu, Spline Finite Strip Method in Structural Analysis, Proceedings, The International Conference on Finite Element Method, Shanghai, pp 704-709, 1982.

[27] Mizusawa, Tomisaku, Application of Spline Strip Method to Analyze Vibrations of Open Cylindrical Shells, Int. J. Num. Meth. Engng., 26, pp 663-676, 1988.

[28] Mendelson, A., Plasticity, Theory and Application, The MacMillan Company, New York, 1968.

[29] Zienkiewicz, O.C., The Finite Element Method in Structural and Continuum Mechanics, Mcgraw Hill Publishing Company, London, 1968. 
[30] Wu, R.W.H. and Witmer, E.A., Non-linear Transient Responses of Structures by the Spatial Finite Element Method, AIAA Journal Vol. 11, No.8, pp. 11101117, 1973.

[31] Scordelis, A.C. and K.S.Lo, Computer Analysis of cylindrical shells, J. Am. Concr. Inst. 61, pp 539-561, 1964.

[32] Cowper, G.R., G.M.Lindberg and M.D.Olson, A Shallow shell finite element of triangular shape, Int. J. Solids Structures, Vol.6, pp. 1133-1156, 1970.

[33] Bergan, P.G., G.Horrigmoe, B.Krakeland and T.H.Soriede, Solution Techniques for Non-Linear Finite ELement Problems, Int. J. Num. Meth. Engng., Vol.12, pp. 1677-1696, 1978.

[34] Allman, D.J., A Compatible Triangular Element including Vertex Rotations for Plane Elasticity Analysis, Computers and Structures, Vol. 19, pp. 1-8, 1984.

[35] Razzaque, A. and B. M. Irons, A Further Modification to Ahmad's Shell Element, Int. J. Num. Meth. Engng., Vol. 5, No.4, pp. 588-589, 1973.

[36] Noor, A.H., Recent Advances in Reduction Methods for Non-Linear Problems, Computers and Structures, Vol.23, pp. 31-44, 1981.

[37] Hughes, T.J.R., R.L.Taylor and W.K.Nukulachai, A Large Deformation Formulation for Shell Analysis by the Finite Element Method, Computers and Structures, Vol. 13, pp. 19-27, 1981.

[38] Olson, M.D. and T.W. Bearden, A Simple Flat Triangular Shell Element Revisited, Int. J. Num. Meth. Engng., Vol. 14, No. 1, pp. 51-68, 1979.

[39] Gould, P.L. Analysis of Shells and Plates, Springer-Verlag, New York, 1988.

[40] Abayakoon, S.B.S., M.D.Olson and D.L.Anderson, Large Deflection ElasticPlastic Analysis of Plate Structures by the Finite Strip Method, Int. J. Num. 
Meth. Engng., Vol. 28, pp. 331-358, 1989.

[41] Heppler, G.R., On the Analysis of Shell Structures subjected to a Blast Environment: A Finite Element Approach, Report No. 302, UTIAS, 1986.

[42] Kempner, J, Unified Thin-Shell theory, Symposium on the Mechanics of Plates and Shells for Industrial Resenrch Associates, Polytechnic Institute of Brooklyn, PIBAL no. 566, 1960.

[43] Baker E.H., L. Kovalevsky and F.L. Rish, Structural Analysis of Shells, McGraw Hill Book Company, New York, 1972.

[44] Donell, L.H., Simplified Shell Theory, NACA Report 479, 1933. 